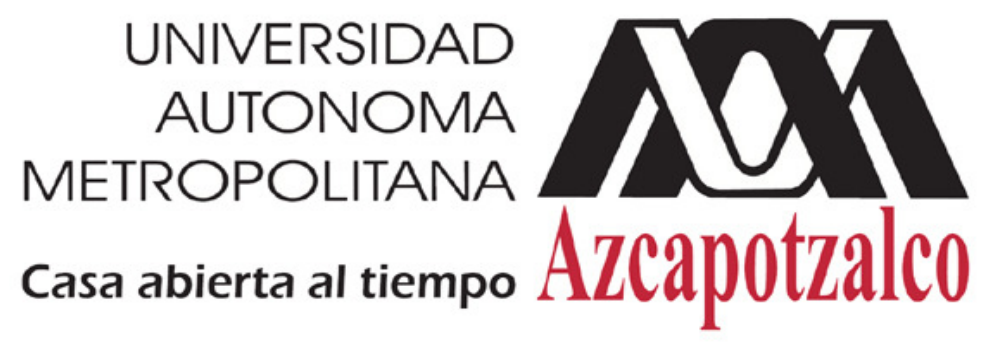

Ciencias Básicas e Ingeniería

\title{
Construcción y caracterización de un cristal artificial elástico
}

\section{Tesis de Doctorado}

en

Ciencias e Ingeniería de los Materiales presenta:

M. en C. Filiberto Ramírez Ramírez

Asesor:

Dra. María Gabriela Báez Juárez

Universidad Autónoma Metropolitana, Azcapotzalco 


\section{Índice general}

Agradecimientos $\quad$ IV

$\begin{array}{lll}\text { Abstract } & \text { VI }\end{array}$

Resumen

Lista de acrónimos $\quad$ VIII

$\begin{array}{ll}\text { Objetivos } & 1\end{array}$

1. Introducción $\quad 2$

2. Marco teórico $\quad 5$

2.1. Cristales ....................... . . . 5

2.1.1. Cristales en la naturaleza . . . . . . . . . . . . . . . 5

2.1.2. Cristales artificiales finitos . . . . . . . . . . . . . . 6

2.1.3. Estructuras de bandas . . . . . . . . . . . . . . . . . 8

2.1.4. Cristales elásticos . . . . . . . . . . . . . . . . . . . 11

2.2. Modelo de enlace fuerte en mecánica cuántica . . . . . . . . . . . . . . 13

2.3. Modelo de enlace fuerte en mecánica clásica . . . . . . . . . . . . . . 15

2.4. Modelo de enlace fuerte en microondas . . . . . . . . . . . . . . . . . . . . 17

2.5. Modelo de enlace fuerte en elasticidad . . . . . . . . . . . . . . . . . 19

3. Diseño de un cristal elástico artificial usando matriz de transferencia 22

3.1. Vibraciones torsionales en una varilla simple . . . . . . . . . . . . . . 22

3.2. Sistema elástico localmente periódico . . . . . . . . . . . . . . . . . . 24

3.2.1. Determinación del parámetro $\eta$. . . . . . . . . . . . . 25

3.2.2. Determinación del valor del parámetro $\epsilon$. . . . . . . . . . . 27

3.2.3. Determinación de la sección transversal del sistema . . . . . . . . . 28

3.3. Sistema elástico localmente periódico diseñado . . . . . . . . . . . . . . . . 29

3.4. Sistema localmente periódico con un defecto . . . . . . . . . . . . . . . 32

3.5. Sistema localmente periódico con dos defectos . . . . . . . . . . . . . . 35

3.6. Sistema localmente periódico con varias superceldas acopladas . . . . . . . 39 
4. Diseño numérico del cristal elástico usando el modelo de acoplamiento elástico

4.1. Modelo de enlace fuerte elástico empírico para describir el cristal elástico diseñado . . . . . . . . . . . . . . . . . . 43

4.1.1. Acoplamiento de 2 superceldas elásticas . . . . . . . . . . . . . 46

4.2. Generalización del modelo de enlace fuerte elástico para un cristal infinito 52

5. Resultados experimentales $\quad 57$

5.1. Fabricación de los sistemas elásticos estructurados . . . . . . . . . . . 57

5.2. Técnica de espectroscopia acústica resonante (ARS) . . . . . . . . . . . 59

5.3. Resultados experimentales . . . . . . . . . . . . . . . . . 61

5.3.1. Espectro de frecuencia . . . . . . . . . . . . . . . 61

5.3.2. Amplitud de onda. . . . . . . . . . . . . . . . 63

$\begin{array}{lr}\text { 6. Conclusiones } & 69\end{array}$

$\begin{array}{ll}\text { Bibliografía } & 71\end{array}$

$\begin{array}{ll}\text { A. Matriz de transferencia } & \mathbf{7 5}\end{array}$

A.1. Matriz de transferencia . . . . . . . . . . . . . . . . . . . 75 
A mis padres Rufina y Rodrigo, a mis hermanos Rodrigo, Fatima, Maria y Juan, a mis abuelitos Leon y Petra, gracias por todo su apoyo incondicional y por su infinita confianza. Siempre estan en mi mente y en mi corazón. 


\section{Agradecimientos}

Agradezco al Posgrado en Ciencias e Ingeniería de los Materiales de la UAM-Azcapotzalco, por haberme dado la oportunidad de formar parte de sus filas.

Mi gratitud a la Dra. María Gabriela Báez Juárez por haber aceptado ser mi asesora de la licenciatura, maestría y ahora del Doctorado. Gracias por haberme ayudado a subir el último peldaño de mi carrera profesional. Toda una decada de trabajo duro. Un agradecimiento especial al Dr. Rafael Alberto Méndez Sánchez quien me permitió hacer una estancia de 2 años en el Centro Internacional de Ciencias (CIC) y quien me ayudo a la parte experimental, numérica y análitica de la tesis. A la maestra Eliza, quien me ayudo con la elaboración y conclusión de la tesis, al Dr. Moises por su apoyo incondicional, al Dr. Miztli por su apoyo en la parte análitica de mi trabajo. A mis compañeros de trabajo, Enrique y Arturo, a quienes conosco desde la licenciatura y quienes me apoyaron en la ejecución de mi proyecto terminal. Agradezco a mis compañeros de trabajo Milton, Denis y en general a todos los integrantes del Grupo de Ondas y Materiales por parte de la UAM-A y de la UAM-I.

Este trabajo no se podria haber realizado sin la beca proporcionada por CONACYT, con número (CVU) 502119, además de los apoyos brindados por CONACYT mediante los proyectos 284096 (átomos artificiales elásticos) y AI-S-33920 y por parte de DGAPA con el proyecto IN103115. Quiero agradecerles a todas estas instancias que hicieron posible este gran proyecto.

Agradezco al CIC por haberme permitido realizar los experimentos dentro de sus instalaciones. Agradezo al Ingeniero Hector Gálvan por haber construido el sistema a estudiar. Agradezco a mis compañeros de trabajo al Dr. Antonio, al Dr. Victor y al Dr. Angel, quienes siempre estuvieron muy atentos del curso del trabajo. Asi como también agradezco al M en Ciencias Mario, al Ing. Diego Camacho y al Ing. Esteban. 


\section{Abstract}

The objective of this research is the design, manufacture and characterization of a quasi-one-dimensional elastic artificial crystal, with spectral properties similar to those of an atomic crystal. To do this, the "unit elastic supercell" was first numerically designed using the transfer matrix method (MMT). The latter gave rise to the periodic structure of an aluminum rod which we call: artificial elastic crystal, because its torsional frequency spectrum is characterized by allowed bands whose phenomenology obeys a strong bond model. The wave amplitudes associated with these bands are localized, in analogy with a one-dimensional atomic crystal. The elastic structure obtained corresponds to a system of elastic resonators, coupled together by a locally periodic structure. This numerical design allowed the fabrication of five structured aluminum rods corresponding to two isolated elastic supercells and up to six coupled supercells. Each of these systems were characterized in the laboratory to measure both their torsional frequency spectra and some of their own wave amplitudes. In the $26300-26600 \mathrm{kHz}$ frequency range, the existence of the first allowed band of the manufactured artificial crystal was verified, with an error of less than $1 \%$ with respect to the theoretical predictions. An empirical strong bond model is proposed for the torsional waves in this elastic artificial crystal with two free parameters. This model significantly reduces numerical simulations to obtain the frequency spectrum of the system with respect to MMT and finite elements and is one of the main contributions of this work. 


\section{Resumen}

El objetivo de esta investigación es el diseño, fabricación y caracterización de un cristal artifical elástico cuasi-unidimensional, con propiedades espectrales similares a las de un cristal atómico. Para ello, primero se diseño nuéricamente la "supercelda elástica unitaria' ' mediante el método de la matriz de transferencia (MMT). Esta última, dio origen a la estructura periodica de una varilla de aluminio a la que llamamos: cristal elástico artificial, debido a que su espectro de frecuencias torsionales se caracteriza por bandas permitidas cuya fenomenología obedece un modelo de enlace fuerte. Las amplitudes de onda asociadas a estas bandas están localizadas, en analogía con un cristal atómico unidimensional. La estructura elástica obtenida, corresponde a un sistema de resonadores elásticos, acoplados entre sí por una estructura localmente periódica. Este diseño numérico permitio la fabricación de cinco varillas de aluminio estructuradas correspondientes a dos superceldas elásticas aisladas y hasta seis superceldas acopladas. Cada uno de estos sistemas se caracterizaron en el laboratorio para medir, tanto sus espectros de frecuencia torsional, como algunas de sus amplitudes de onda propias. En el intervalo de frecuencias de 26300-26600 $\mathrm{kHz}$, se comprobo la existencia de la primera banda permitida del cristal artificial fabricado, con un error menor al 1\% respecto a las predicciones teóricas. Se propone un modelo empírico de enlace fuerte para las ondas torsionales en este cristal artificial elástico con dos parámetros libres. Este modelo reduce notablemente las simulaciones numéricas para

obtener el espectro de frecuencias del sistema respecto a MMT y elementos finitos y es una de las principales contribuciones de este trabajo. 


\section{Lista de acrónimos}

ARS: espectroscopía acústica resonante.

TMM: método de la matriz de transferencia.

EMAT: transductor electromagnético acústico.

TB model: modelo de Tight Binding.

LCAO: combinación lineal de orbitales atómicos.

SC: superceldas.

Fresadora CNC: Fresadora con control numérico por computadora.

VNA: Analizador de redes vectorial. 


\section{Objetivos}

\section{Objetivo general}

Mostrar que se pueden construir sistemas elásticos acoplados cuyas vibraciones, medidas en el laboratorio, pueden ser descritas adecuadamente con modelos de enlace fuerte.

Objetivos específicos

- Diseñar un sistema elástico ondulatorio, descrito por un modelo de enlace fuerte, basado en un arreglo de unidades elásticas acopladas. Se construirá dicho sistema con las dimensiones apropiadas para que pueda ser caracterizado por espectroscopía acústica resonante (ARS).

- Generar un programa basado en el modelo teórico propuesto, que simule las vibraciones de un sistema de unidades elásticas acopladas.

- Identificar los modos de vibración elásticos del sistema cuya respuesta al acoplamiento reproduzca mejor un modelo de enlace fuerte.

- Usando la simulación numérica determinar el número mínimo de celdas elásticas que muestre propiedades del sistema localmente periódico.

- Caracterizar el sistema acoplado con unidades elásticas por ARS.

- A partir del modelo teórico y la simulación numérica construir un cristal artificial elástico unidimensional.

- Diseñar un método eficiente de acoplamiento entre unidades elásticas resonantes que muestre características del llamado enlace fuerte.

- Identificar los parámetros relevantes del sistema elástico acoplado para hacer la anología con el modelo de enlace fuerte. Cavidad resonante y acoplamiento.

- Caracterizar por ARS el sistema elástico fabricado y comparar con el modelo teórico propuesto. 


\section{Capítulo 1}

\section{Introducción}

Actualmente el estudio experimental de sistemas elásticos ondulatorios se encuentra desfasado con respecto a la gran cantidad de investigaciones teóricas y numéricas reportadas en la literatura. Esto se debe a los grandes retos por resolver que se presentan en el laboratorio debido a la naturaleza tensorial de las ondas elásticas, ya que existen múltiples acoplamientos entre las distintas polarizaciones. Además existe la conversión de unas a otras al reflejarse en la interfase entre dos medios distintos. Sin embargo, al resolver estos retos de laboratorio, se abre una robusta línea de investigación experimental enfocada a la caracterización y diseño de materiales elásticos, basada en sus propiedades de transporte ondulatorio mecánico. Así, es posible diseñar análogos de otros sistemas ondulatorios clásicos tales como cavidades de microondas y sistemas acústicos, entre otros. Este tipo de investigación ha dado origen a nuevos materiales artificiales elásticos que controlan la propagación de ondas mecánicas por medio de estructuras estrategicas y que se encuentran dentro de los llamados metamateriales elásticos.

Entre los muchos estudios teóricos y numéricos [1, 2, 3, 4, 5] sobre sistemas elásticos ondulatorios podemos mencionar el trabajo de la referencia [5] donde presentan las figuras de Chladni de los primeros 6 modos en una placa y obtienen una concordancia sobresaliente entre todos los patrones nodales obtenidos análiticamente con los trabajos experimentales de otros investigadores.

Por su parte, se han reportado estudios teóricos y experimentales de vibraciones en varillas con diversas estructuras [6]. Algunos resultados que destacan de estos trabajos son: el surgimiento de bandas en el espectro de frecuencias, correspondientes a sistemas localmente periódicos y la medición de sus amplitudes de onda. Estos resultados se lograron incorporando al montaje experimental, de espectroscopia acústica resonante (ARS, por sus siglas en inglés), los transductores electromagnéticos acústicos tipo EMAT (por sus siglas en inglés)[7]. Estos dispositivos han permitido resolver algunos de los retos experimentales y son usados para excitar selectivamente las distintas polarizaciones de las ondas elásticas en materiales paramagnéticos, así como para registrar la respuesta elástica del material a dichas excitaciones. El principio de funcionamiento de dichos dispositivos es la ley de inducción de Faraday. Así, se pueden generar preferentemente distintos tipos de vibraciones en los materiales elásticos. Particularmente en sistemas cuasi-unidimensionales como varillas, donde se pueden generar vibraciones compresionales, torsionales y flexiona- 
les. Esto se logra gracias a las configuraciones que favorecen la exitación de cada tipo de ondas mecánicas en el sistema, por medio de la reducción de los acoplamientos que existen entre ellas. Entre las ventajas que implica el uso de estos transductores podemos contar las siguientes: 1.- Son fáciles de construir con componentes comerciales, 2.- Su costo es muy bajo, 3.- Son altamente selectivos a los diferentes tipos de polarización mecánica en configuraciones estrategicas, 4.- Son dispositivos reversibles, lo cual significa que pueden funcionar como excitadores y detectores de vibraciones mécanicas y 5.- No tienen contacto físico con el sistema, lo cual permite un mayor control de la frecuencia de la señal excitadora.

La versatilidad de estos transductores ha facilitado diversas investigaciones, como por son: la primera medición del coeficiente de Timoshenko [8]; la obtención de la estructura de bandas, en varillas con estructura periódica [6]; la comprobación de algunas predicciones de Navier [9] y la comprobación de la existencia del segundo espectro de Timoshenko [10], entre otros. Tambén se han usado los EMATS para caracterizar por la técnica de ARS sistemas simples [11]; y sistemas estructurados, en 1D [6] y en 2D; sistemas periódicos bidimensional así como la medición de la estructura de bandas [12]; medición del transporte elástico ondulatorio (ondas mecánicas) a tráves cavidades caóticas elásticas [13] y la medición de estados atrapados elásticos en guías de ondas dobladas [14].

En esta investigación se desarrolla un mecanismo alternativo para generar estados atrapados en sistemas elásticos, usando estructuras cristalinas deformadas. El mecanismo consiste en usar las brechas del espectro de una estructura cristalina dentro de la cual se generan nuevas bandas emergentes asociadas a los estados de celdas unitarias deformadas (defectos). Los estados correspondientes a estas bandas emergentes obedecen a un modelo de enlace fuerte elástico, cuyas amplitudes de onda estan localizadas. Es bien sabido que un sistema périodico se caracteriza por un espectro con estructuras de bandas y brechas [6], ya sea de energía o de frecuencia. Por ello, al introducir celdas deformadas (defectos) en la estructura periódica original sus amplitud de onda se localizan alrededor de la posición del defecto, decayendo exponencialmente hacia los extremos. Este es un resultado ya reportado en sistemas elásticos estructurados, que usaremos en este trabajo para construir un material con transporte evanescente de dichos estados localizados [15].

El sistema de celdas elásticas acopladas que aquí se propone, satisfacen un modelo de enlace fuerte análogos a los conocidos en mecánica cuántica. Este modelo elástico no es trivial ya que una estructura elástica con periodicidad simple no satisface el modelo de enlace fuerte.

En el siguiente capítulo se tratan algunos conceptos básicos del modelo de enlace fuerte aplicado a los diferentes sistemas y se hace una revisión de los diferentes tipos de cristales. En el capítulo 3 se estudia un sistema localmente periódico con una hasta seis impurezas igualmente espaciadas y se logra obtener una banda de frecuencias, asociadas a las impurezas, dentro de la brecha. En el mismo capítulo se analiza el comportamiento de la amplitud de onda asociado a una impureza y se determina que tiene un decaimiento exponencial en la posición de la impureza. En el capítulo 4 se desarrolla un modelo de Tight-Binding usando la propiedad de localización como un estado base del modelo. En ese mismo capítulo se muestra que las frecuencias de la banda emergente de los sistemas 
bajo estudio están distribuidas simétricamente alrededor de la frecuencia de una impureza aislada, este hecho es en sí mismo un resultado importante ya que reproduce en un sistema elástico la fenomenología de un cristal atómico unidimensional. En el capítulo 5 se describe la configuración experimental y se detallan los resultados que muestran la aparición de una banda de transmisión para sistemas con 2 y hasta 6 impurezas. También se muestra una excelente concordancia de nuestros experimentos con el modelo de Tight-Binding. En el capítulo 6 presentamos brevemente nuestras conclusiones. 


\section{Capítulo 2}

\section{Marco teórico}

En este capítulo mostramos la gran versatilidad del modelo de enlace fuerte. Entre los sistemas en que se ha utilizado este modelo se encuentran sistemas cuánticos [16, 17], clásicos [18], de microondas [19, 20,21] y acústicos [22, 23, 24, 25, 26, 27]. Se explica tanto el modelo de enlace fuerte para un cristal unidimensional como la ecuación que caracteriza el modelo de enlace fuerte independiente del tiempo. De manera ilustrativa se muestra la relación de dispersión de una cadena unidimensional de masas idénticas acopladas mediante fuerzas lineales y también para un conjunto de $\mathrm{N}$ péndulos acoplados por resortes. Además se muestra el estudio, con enlace fuerte, de la propagación de microondas a tráves de 3 sistemas, uno en forma de grafeno y dos más en forma de una cadena. Por último se muestra un sistema penrose hecho con diapasones. Antes de comenzar es importante recordar la definición de los cristales y sus propiedades fundamentales.

\subsection{Cristales}

\subsubsection{Cristales en la naturaleza}

En la vida cotidiana estamos rodeados de cristales como el azúcar que empleamos en la cocina, entre otros. En estado sólido un cristal es un arreglo infinito ordenado de átomos, iones o moléculas, dispuestos en forma regular, el cual da lugar a estructuras periódicas con simetrías particulares. Además un cristal posee caras planas bien definidas; así como aristas rectas y vértices agudos. Para determinar las propiedades físicas y químicas de un cristal es necesario determinar su estructura, siendo esta de geometría regular. Algunos ejemplos de cristales son el cuarzo y la sal común (ver figura 2.1). 




Figura 2.1: Izquierda: Aspecto físico de un cristal de cuarzo; derecha: Aspecto físico de un cristal de sal

\subsubsection{Cristales artificiales finitos}

\section{Cristales fotónicos}

Además de los cristales que existen en la naturaleza, se pueden construir estructuras artificiales altamente ordenadas (periódicas) como son los cristales fotónicos [28] (ver figura 2.2).
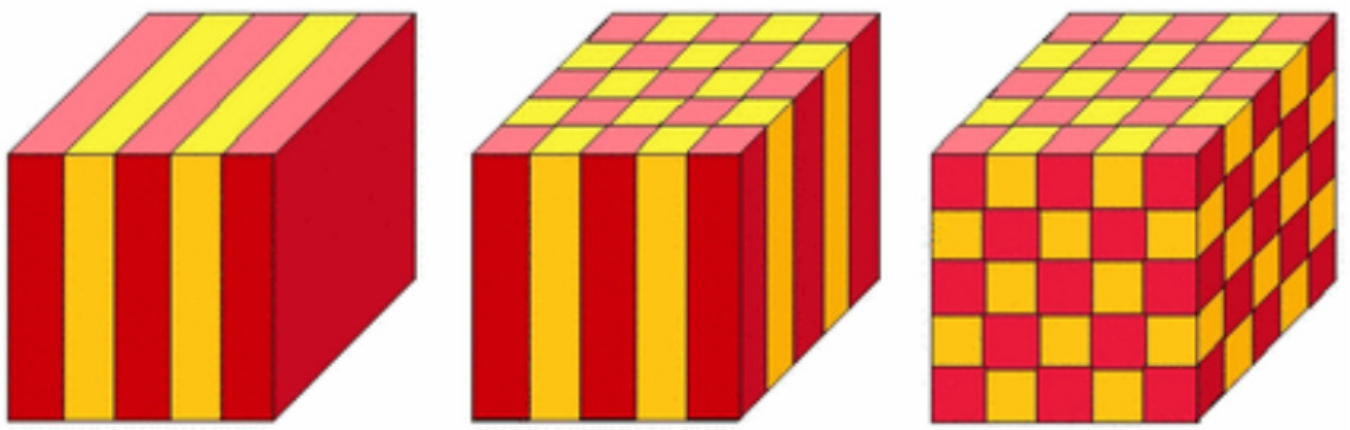

Figura 2.2: Estructuras fotónicas en 1, 2 y 3 dimensiones, formadas por la alternancia de capas de dos materiales con distinta función dieléctrica (en rojo y amarillo respectivamente), superpuestos.

Se define un cristal fotónico como un arreglo regular compuesto de materiales con diferentes índices de refracción. Cuando la variación de la función dieléctrica es a lo largo de una dirección espacial se tiene un cristal fotónico en una dimensión (1D). En el caso en el que la variación se da a lo largo de dos y tres direcciones espaciales independientes, los cristales correspondientes fotónicos son en 2 y 3 dimensiones (2D) y (3D), respectivamente. El principio de funcionamiento de los cristales fotónicos, es muy similar a los de los semiconductores; es decir, tienen un intervalo de bandas de frecuencias que permiten 
que se propaguen en todas las direcciones las ondas electromagnéticas; mientras que en otras se inhiben. Los cristales fotónicos en 1D tienen propiedades ópticas bien conocidas. Por ejemplo, se utilizan como espejos de alta reflexión y revestimiento antirreflectante. En los últimos años, la mayoría de las investigaciones relacionadas con el control del campo de radiación se han concentrado en cristales fotónicos $2 \mathrm{D}$ y $3 \mathrm{D}$, ya que ofrecen una rica variedad de nueva física en dicha área. Si se diseñan correctamente los cristales fotónicos 3D, es posible que aparezca un rango de frecuencia donde no existe un modo propio electromagnético. Los rangos de frecuencias de este tipo se denominan intervalos de banda fotónica, ya que corresponden a bandgaps de estados propios electrónicos en cristales comunes, pero si se introduce un desorden en la estructura dieléctrica regular del cristal fotónico, podemos obtener los modos midgap cuyas eigenfunciones están localizadas fuertemente alrededor del desorden. A estos modos se les conoce como modos de defecto localizados.

\section{Cristales fonónicos}

Mientras que en un cristal fotónico la permitividad varía periódicamente en el espacio, en un cristal fonónico [29] (ver figura 2.3) las variables que varían macroscopicamente son los coeficientes elásticos. De la misma forma se pueden introducir defectos puntuales o lineales, como cavidades o guías de onda en el cristal fonónico mediante la eliminación de uno, unos pocos o una fila del material. Dependiendo de su geometría y constitución, el efecto de tales defectos pueden subir nuevos modos dentro de la brecha del cristal fonónico; dichos modos corresponden a las ondas localizadas o evanescentes con un desplazamiento decreciente lejos del defecto. Este efecto es muy importante pues dicho material se puede usar para confinamiento y guía de las ondas acústicas; mientras el acoplamiento entre una guía de onda y una cavidad puede ser usado como un dispositivo de filtrado.
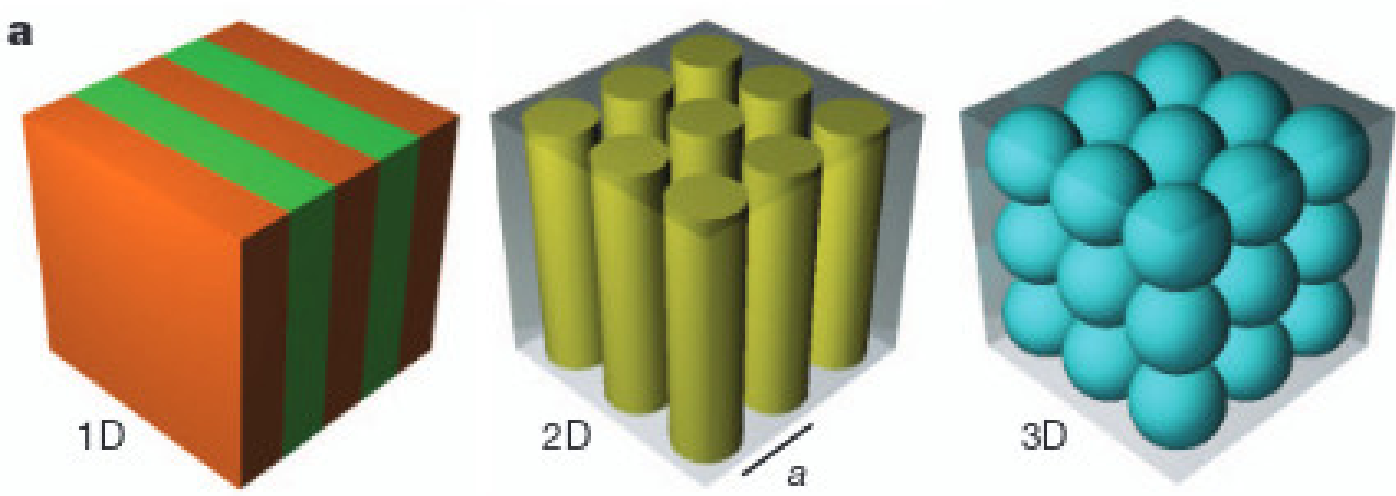

Figura 2.3: Cristales fonónicos en 1D, 2D y 3D hechos de dos materiales diferentes arreglados periodicamente. Los diferentes colores representan materiales con diferentes propiedades elásticas [30]. 


\subsubsection{Estructuras de bandas}

Tanto los cristales naturales como los cristales artificiales y sistemas periódicos, en general, se caracterizan por tener un espectro de energías y/o frecuencias con una estructura de bandas y brechas, como la que se muestra en la figura 2.4

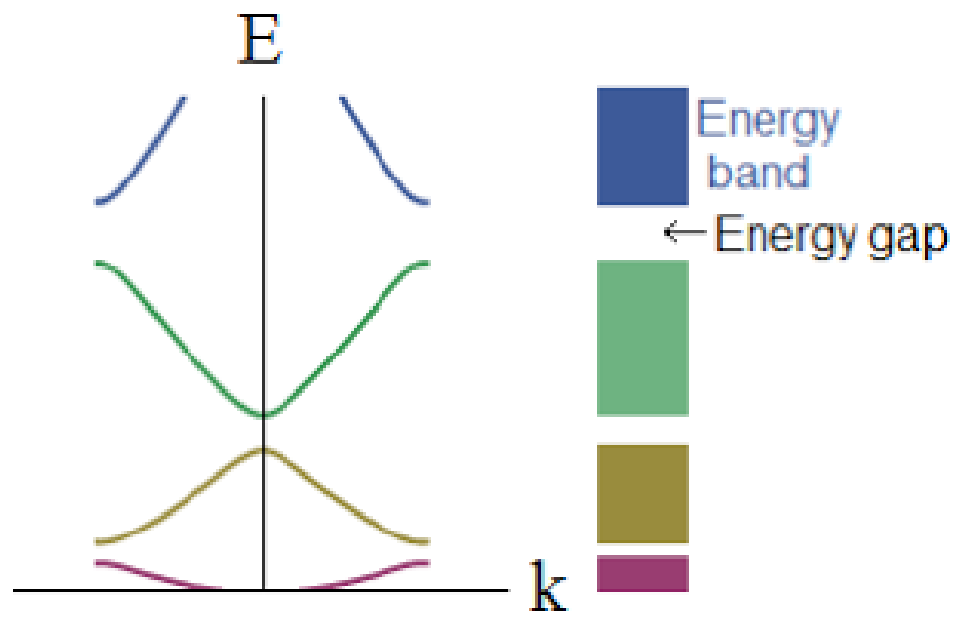

Figura 2.4: Esquema del espectro de energías, asociado a un cristal atómico unidimensional, como función del número de onda k. Una proyección de las curvas sobre el eje de las energías, muestra las bandas y brechas globales en el sistema.

Pero ¿como se forman estas estructuras de bandas?, consideremos primero una disposición periódica de los núcleos iónicos en una red cristalina, donde el potencial iónico sigue la periodicidad del sólido. Para los electrones en un potencial periódico una onda electrónica experimenta la reflexión de Bragg; por lo tanto, se considera un vector de red recíproca $G=2 \pi / a$, con $a$ la constante de red. Entonces el vector de onda en un cristal periódico está definido por $2 \pi / \lambda \pm 2 \pi n / a$, con $n$ un número entero y $\lambda$ la longitud de onda, consecuentemente el vector de onda en un potencial periódico no es único, pero sólo consideraremos el más pequeño, para $n=1$; es decir, en el intervalo $-\pi / a<k<\pi / a$. Tomando las simetrías de la red y la variación de la constante de la red $a$ a lo largo de las direcciones de alta simetría, el volumen más pequeño que contiene todos los vectores de onda se define como la primera zona de Brillouin.

Otra cosa a considerar para entender de donde provienen las estructuras de bandas es el teorema de Bloch, el cual nos dice que $\psi_{k}(\mathbf{r})=e^{i \mathbf{k} . \mathbf{r}} u_{j, \mathbf{k}}$; es decir, tenemos una función de onda $\psi_{k}(\mathbf{r})$ que es igual a una onda plana $e^{i \mathbf{k} . \mathbf{r}}$ con vector de onda $\mathbf{k}$ dentro de la zona de Brilloin por una función $u_{j, \mathbf{k}}$ con la periodicidad de la red. La ecuación anterior nos da una idea de la estructura de banda electrónica. Ahora vamos a considerar un potencial periódico, veremos que dará lugar a bandas y brechas. Habiendo hablado del Teorema de Bloch, ahora podemos introducir el concepto de estructura de bandas. Cuando alguien se refiere a dicho concepto, generalmente va de la mano de su dispersión electrónica, 
$E(\mathbf{k})$; es decir, como la energía de un electrón varía como función del vector de onda $\mathbf{k}$ del cristal. Gracias a que el teorema de Bloch es muy general y que puede aplicarse a cualquier interacción periódica, no solo a los electrones en un potencial periódico causado por la disposición periódica de los iones en una red. Por ejemplo la teoría de bandas se ha aplicado a los fotones en medios diélectricos periódicos para estudiar la estructura de banda fotónica y a su vez la relación de dispersión de los fotones en un cristal fotónico.

\section{Estructuras de bandas de cristales fotónicos}

La búsqueda de band gaps en cristales fotónicos ha sido muy importante debido a sus múltiples aplicaciones, como el control de la propagación de la luz; de ahí la importancia de optimizar el tamaño del band gap. En trabajos recientes han propuesto varios diseños de cristales fotónicos; tal es el caso de un cristal fotónico bidimensional con estructura hexagonal, tipo panal, como se aprecia en la figura 2.5. A través de ondas planas hicieron un riguroso estudio de dicho cristal donde optimizaron el tamaño del band gap (ver figura 2.6) para controlar la propagación de la luz. Para Ta-I Weng y colaboradores esto fue un gran logro ya que concluyen que al hacer la brecha más grande se puede controlar la dirección de la luz más facilmente.

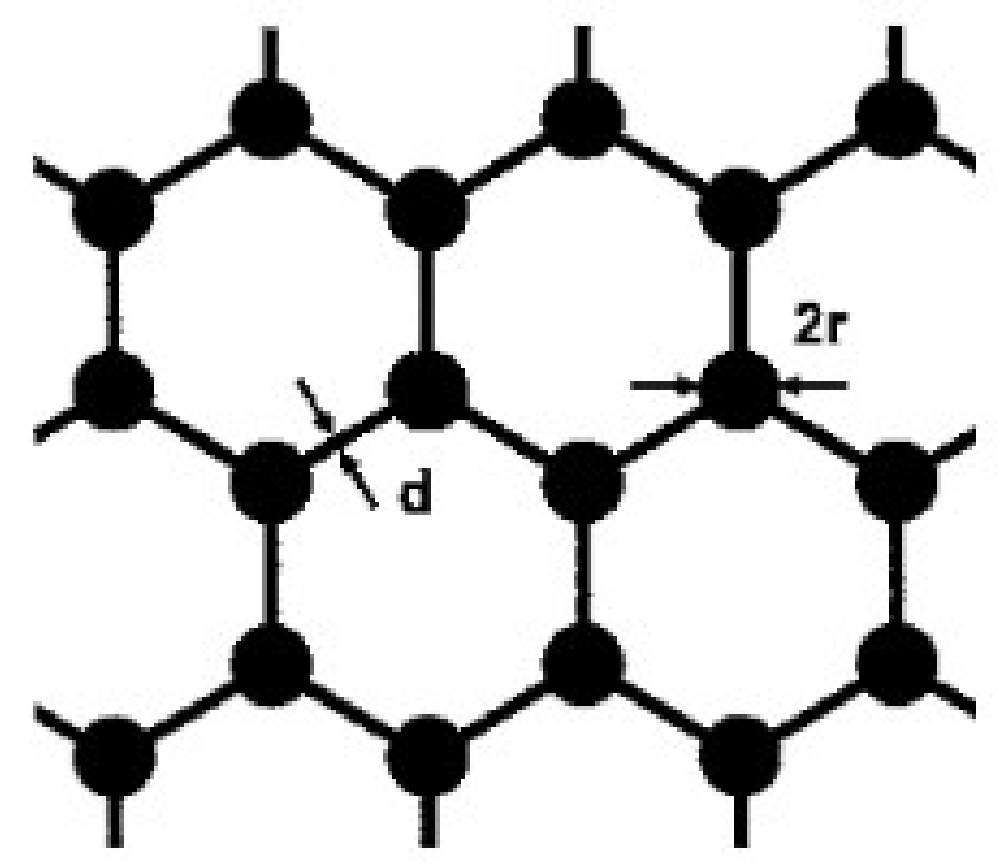

Figura 2.5: Estructura cristalina fotónica bidimensional (2D) en forma de panal compuesta de resonadores cilíndricos acoplados: $\mathrm{r}$ es el radio de los cilindros y d es el espesor de las paredes que conectan a los cilindros [31]. 


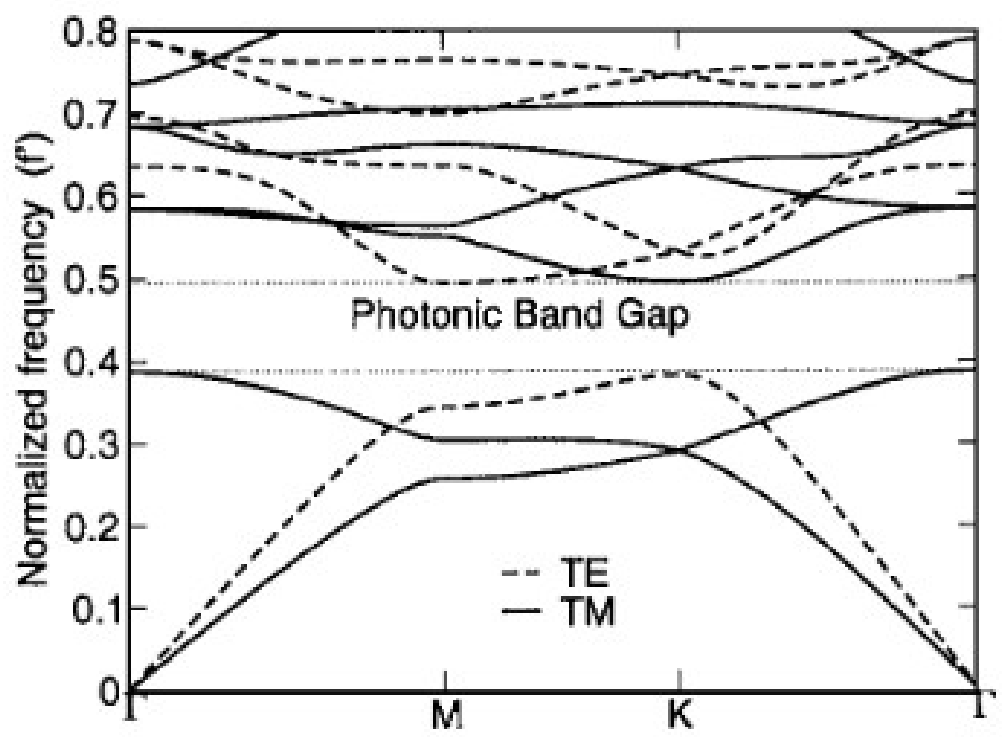

Figura 2.6: Estructura de bandas del cristal fótonico con estructura en forma de panal. Las líneas punteadas horizontales denotan la región de separación de las bandas [31]. Con TE y TM como las polarizaciones del campo eléctrico y magnético, respectivamente y con $\Gamma, M$ y $K$ como los puntos de alta simétria.

\section{Estructura de bandas de cristales fonónicos}

Los cristales fonónicos han sido ampliamente estudiados porque ellos exhiben band gaps donde sólo hay ondas evanescentes mecánicas y no las hay propagantes. Los band gaps fonónicos son similares a los band gaps electrónicos y fotónicos. Esto hace que tengan muchas aplicaciones tales como aislamiento de vibraciones, barreras acústicas, filtros, dispositivos de supreción de ruido, escudos de sonidos, diodos acústicos, metamateriales acústicos y elásticos. Recientes investigaciones han estudiado la estructura de banda elástica (ver figura 2.7) de cristales fonónicos de nanoestructuras circulares de carbono dentro de una red triangular de periodicidad bidimensional. Usando ondas planas encontraron una estructura de banda con una brecha completa muy amplia. 




Figura 2.7: En la figura se muestra la estructura de bandas asociada a una red triangular con nanotubos de carbono con sección transversal circular [32]. En la parte superior derecha se muestra la sección transversal de la celda unitaria. Dicha celda se distribuye periódicamente en una red triangular.

\subsubsection{Cristales elásticos}

A continuación se muestra un ejemplo más de un cristal artificial, un cristal elástico que ya ha sido estudiado. En la referencia [6] los autores estudian el surgimiento de bandas y brechas en el espectro de ondas torsionales de una estructura periódica cuasiunidimensional con una celda unitaria simple (ver figura 2.8), en función del número de celdas. En la gráfica se comparan los valores teóricos y experimentales que están dados para cada celda de izquierda a derecha respectivamente. Dicho sistema artificial elástico se forma por la ranuración periódica a partir de una barra uniforme de una sola pieza con sección transversal circular. Las brechas y bandas de frecuencia son características del comportamiento cristalino del sistema; sin embargo como se discute a continuación, este caso no es totalmente análogo a un cristal atómico. 



Figura 2.8: Izquierda: Estructura elástica periódica con celda unitaria simple. Derecha: Gráfica que muestra el surgimiento de la estructura de bandas de frecuencia en el sistema elástico como función del número de celdas unitarias del sistema (Tomado de la referencia $[6])$.

En el espectro elástico de la figura 2.8 se observa que, a diferencia del espectro de un cristal atómico (ver figura 2.9), los niveles de las bandas emergentes no se distribuyen alrededor de las frecuencias de resonancia del espectro, correspondiente a una única celda sino que se despliegan en forma creciente a partir de la frecuencia original. Es por este motivo que diseñaremos una nueva celda unitaria.
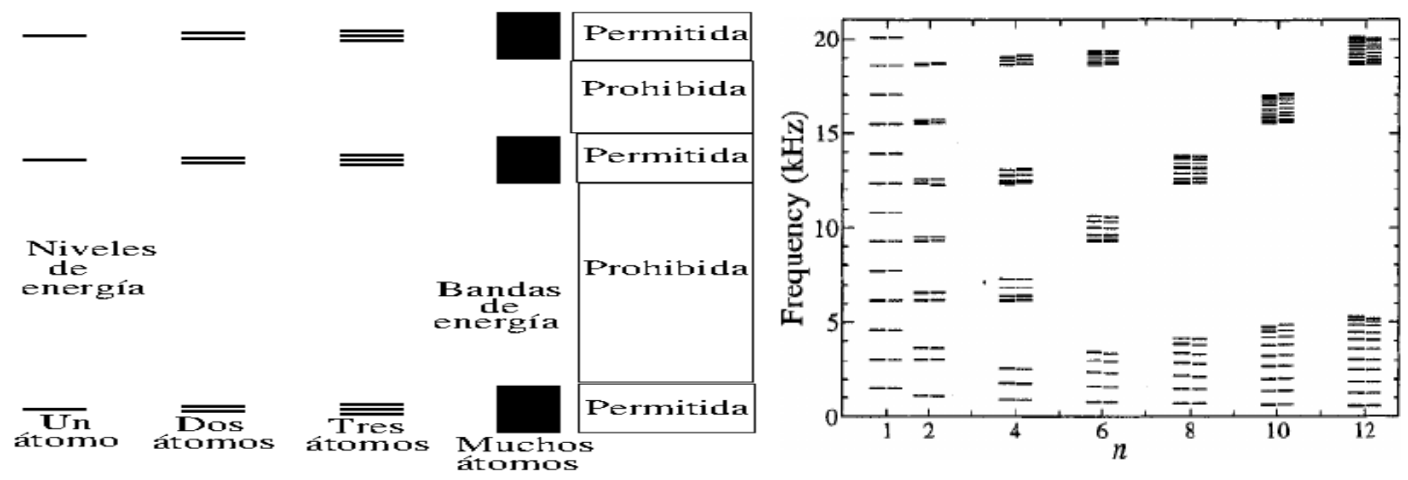

Figura 2.9: Comparación de un espectro de un cristal atómico unidimensional (a la izquierda) con un espectro de un cristal elástico (a la derecha). La distribución de niveles de las bandas, en el primero, es simétrica alrededor del nivel asociado al sistema de un sólo átomo, para el caso de una sola celda. 


\subsection{Modelo de enlace fuerte en mecánica cuántica}

En física del estado sólido, el modelo de Tight-Binding (TB model) conocido como modelo de enlace fuerte, se utiliza como una aproximación para el cálculo de niveles de energía en estructuras cristalinas en general. El modelo de enlace fuerte se ha aplicado con diversos propósitos a distintos sistemas, por ejemplo para el estudio de polímeros conductores, semiconductores orgánicos y electrónica molecular [33], aplicaciones al grafeno (donde se desordena una red por dopaje o por corrugamiento y se observa el cambio de las propiedades de transporte electrónico) [34], la primera realización experimental del oscilador de Dirac, en una dimensión, usando microondas [35] y oscilaciones de Bloch en una dimensión [36]. Este modelo da buenos resultados cualitativos y cuantitativos en muchos casos y puede combinarse con otros modelos, tales como el LCAO (combinación lineal de orbitales atómicos), para mejorar aún más los resultados. El modelo describe las propiedades (energías, frecuencias) de los electrones que están más fuertemente unidos al átomo que pertenecen. El caso más sencillo del modelo de enlace fuerte, describe el comportamiento de un electrón fuertemente ligado a su átomo, dentro de un potencial efectivo; sin embargo, el modelo también proporciona una base para cálculos más complejos, tales como: 1.-Se aplica a diversos tipos de problema de muchos cuerpos [37], 2.cálculo de cuasipartículas [38] y 3.- el cálculo de estados de superficie [39]. En este último se encontró que es posible estudiar las propiedades electrónicas a tráves de un modelo de enlace fuerte. Para esto hay que considerar una base ortogonal de nueve orbitales atómicos, las funciones de Green de superficie e interacciones hasta segundos vecinos.

Un esquema para ayudar a explicar el modelo de enlace fuerte se puede ver en la figura 2.10 considerando el caso más simple, el cual consiste en un cristal unidimensional. Ahí, se observa una serie de átomos alineados horizontalmente e igualmente espaciados entre sí, por una distancia $a$, característica de la malla. Entonces, los átomos se encuentran ubicados en las posiciones $x_{n}=n a$ ( $n$ entero). La eigenfuncion (función de onda) $\phi_{n}$, corresponde al estado de un electrón fuertemente ligado al potencial de un átomo aislado, ubicado en el sitio $n$ de la malla atómica. Note que cada función de onda $\phi_{n}$ se traslapa sólo con las correspondientes a los primeros vecinos, localizadas en las posiciones $n-1$ y $n+1$ respectivamente. Dicho traslape representa, la interacción del átomo $n$ con sus primeros vecinos se puede expresarse en el hamiltoniano del sistema con un potencial constante $V$ que constituye el potencial de acoplamiento interatómico. Esta aproximación permite expandir las funciones de ondas de un sistema de átomos interactuantes, en términos de una base de funciones de onda de un conjunto de átomos aislados, localizados en cada sitio de la malla atómica, considerando únicamente acoplamientos a primeros vecinos (como se muestra en la figura 2.10). Observe que la interacción con los estados y potenciales de los átomos vecinos, es lo que se denomina interacción débil. Como resultado, la función de onda de un electrón sujeto al potencial periódico en el sólido, es similar a la del orbital atómico, en ausencia de interacción con los otros átomos del sólido. Entonces la energía del electrón, en este estado, corresponde a la energía del electrón en el átomo. 


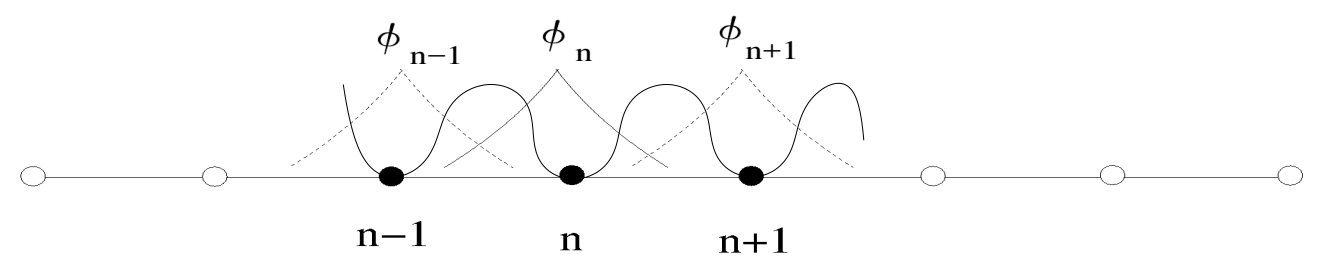

Figura 2.10: Cristal atómico en una dimensión.

La ecuación característica del sistema, de la figura 2.10, se deduce como sigue: considere la ecuación Schrödinger independiente del tiempo del sistema de $N$ átomos interactuantes,

$$
H \Psi_{i}(x)=E_{i} \Psi_{i}(x),
$$

donde $E_{i}$ : es la energía asociada al estado del sistema acoplado de átomos y a la función de onda $\Psi_{i}(x)$ correspondiente. Ésta última puede ser expandida en la base de las eigenfunciones $\left\{\phi_{n}(x)\right\}$ de cada átomo aislado (orbital atómico), localizados en cada sitio $n$ de la malla, así:

$$
\Psi_{i}(x)=\sum_{n} C_{n} \phi_{n}(x)
$$

En esta expansión suponemos conocidas las funciones de onda $\left\{\phi_{n}(x)\right\}$ de la base y deben ser determinados el conjunto de valores $C_{n}$, que corresponden a los coeficientes de la amplitud de onda en el sitio $n$. Con esta finalidad usaremos el procedimiento estándar para evaluar el conjunto de coeficientes $\left\{C_{n}\right\}$ así, sustituimos la expansión (2.2) en (2.1) y multiplicamos por $\phi_{m}^{*}(x)$ e integraremos sobre $x$ ambos lados de la ecuación resultante de manera que obtenemos,

$$
<\phi_{m}^{*}(x)|\hat{H}| \Psi_{i}(x)>=\sum_{n} C_{n} \int \phi_{m}^{*} \hat{H} \phi_{n} d x=\sum_{n} E_{n} C_{n} \int \phi_{m}^{*} \phi_{n} d x .
$$

Separando cada una de las sumatorias en dos partes

$$
\sum_{n \neq m} C_{n} \int \phi_{m}^{*} \hat{H} \phi_{n} d x+C_{m} \int \phi_{m}^{*} \hat{H} \phi_{m} d x=E_{n} \sum_{n \neq m} C_{n} \int \phi_{m}^{*} \phi_{n} d x+E_{m} C_{m} \int \phi_{m}^{*} \phi_{m} d x(2.4)
$$

Aprovechando las propiedades de ortogonalidad de los elementos de la base y aplicando propiedades de ortonormalidad entre el conjunto de funciones $\phi_{n}$ llegamos a

$$
\sum_{n \neq m} C_{n} \int \phi_{m}^{*} \hat{H} \phi_{n} d x+C_{m} \int \phi_{m}^{*} \hat{H} \phi_{m} d x=E_{m} C_{m}
$$


la cual podemos reescribir como,

$$
\sum_{n \neq m} C_{n} V_{m n}+C_{m} \epsilon_{m}=E_{m} C_{m} .
$$

Donde $V_{m n}$ representa el acoplamiento entre orbitales atómicos vecinos y es un valor real, tal que $V_{m n}=V_{m n}^{*}$. Podemos simplificar más la notación de manera que, si los acoplamientos son iguales $V_{m n}=V$, entonces

$$
V_{m n}=\left\{\begin{array}{lll}
V & \text { para } & n=m+1 \\
V & \text { para } & n=m-1 \\
0 & \text { para } & n=m+p \text { con } p= \pm 2 \pm 3 \ldots
\end{array}\right.
$$

y considerando la ecuación (2.7), finalmente la ecuación (2.3) puede reescribirse así,

$$
C_{n+1} V+C_{n} \epsilon_{n}+C_{n-1} V=E C_{n},
$$

donde

$$
\begin{aligned}
V=V_{m n} & =V_{n m}=\int d x \phi_{m}^{*} \hat{H} \phi_{n}, \\
\epsilon_{m} & =\int d x \phi_{m}^{*} \hat{H} \phi_{m} .
\end{aligned}
$$

La ecuación (2.8) caracteriza el modelo de enlace fuerte independiente del tiempo. En ella, $C_{n}$ corresponde a la amplitud de la función de onda en el sitio $n$; $V$, son los elementos de matriz que acoplan orbitales vecinos; $\epsilon_{n}$, es la energía de sitio de cada átomo aislado y por último $E$ son los eigenvalores o energías del sistema interactuante de $n$ sitios acoplados. Este modelo, además de aplicarse a la física atómica, molecular y química para el cálculo del espectro de energías, también es ampliamente utilizado en sistemas ópticos $[40,41,42]$ y de microondas [43, 44, 45]. En sistemas acústicos y elásticos también se ha aplicado $[46,47,48]$, pero aún no hay resultados experimentales que confirmen de manera confiable las predicciones de éste.

\subsection{Modelo de enlace fuerte en mecánica clásica}

El modelo de enlace fuerte también se puede hacer usando una cadena unidimensional de átomos idénticos de masa $\mathrm{m}$, como se muestra en la figura 2.11. Los cuales también podrían ser $\mathrm{N}$ masas acopladas con resortes. 

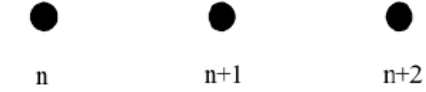

Figura 2.11: Cadena de átomos acoplados mediante fuerzas lineales. Tomado de la referencia [49].

La ecuación de la figura 2.11 está dada por

$$
F_{n}=C\left(u_{n+1}-u_{n}\right)+C\left(u_{n-1}-u_{n}\right) .
$$

También se puede escribir

$$
m \frac{d u_{n}}{d t}=C\left(u_{n+1}+u_{n-1}-2 u_{n}\right),
$$

cuya solución

$$
u_{n}=A e^{i(\omega t-n k a)},
$$

si sustituimos obtenemos la relación de dispersión

$$
m \omega^{2}=2 C(1-\operatorname{cosk} a),
$$

despejando

$$
\omega=\sqrt{\frac{4 C}{m}}\left|\sin \frac{1}{2} k a\right| .
$$

Si graficamos obtenemos la primera zona de Brillouin

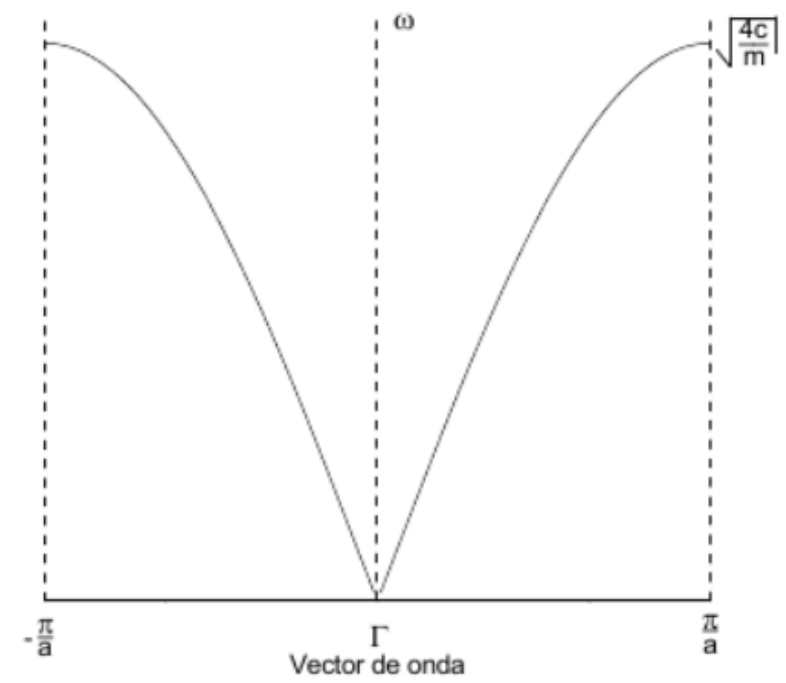

Figura 2.12: Primera zona de Brillouin [49]. 


\subsection{Modelo de enlace fuerte en microondas}

El modelo de enlace fuerte ha sido ampliamente utilizado en sistemas de microondas como se muestra el trabajo experimental de la referencia [50], entre otros. En dicha referencia se construye un modelo empírico que describe el comportamiento de un conjunto de resonadores en forma de discos con alto índice de refracción acoplados entre sí por aire.
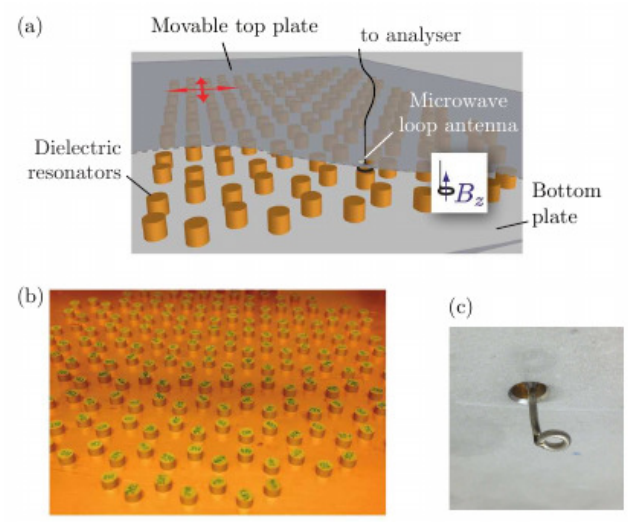

Figura 2.13: (a) Discos con un alto índice de refracción son colocados entre dos placas metálicas (b) imagen de la estructura dieléctrica (c) imagen de la antena [50].

Estos discos se encuentran entre dos placas metálicas (ver figura 2.13) formando un sistema de resonadores acoplados a tráves del cual pasan microondas. El decaimiento de estas ondas, puede ser modulado a través de la posición de los cilindros resonadores. El sistema es analizado mediante el modelo de enlace fuerte para estudiar la propagación de microondas a tráves de un sistema de resonadores; los cuales estan localizados en los nodos de una red hexagonal, en analogía con una red de grafeno, donde las microondas juegan el papel de los electrones en el grafeno. Los autores miden la densidad de estado asociada a cada una de las eigenfrecuencias. Al variar la distancia entre los distintos resonadores, se puede controlar la amplitud de resonancia y ver el efecto que tiene en la densidad de estados. Ellos estudian un sistema con un sólo resonador y un sistema con dos niveles. Al comparar la densidad de estado obtenida experimentalmente con la solución analítica hay una buena predicción, apesar de que su sistema es finito con 220 discos dieléctricos y su modelo análitico esta hecho para sistemas infinitos. 

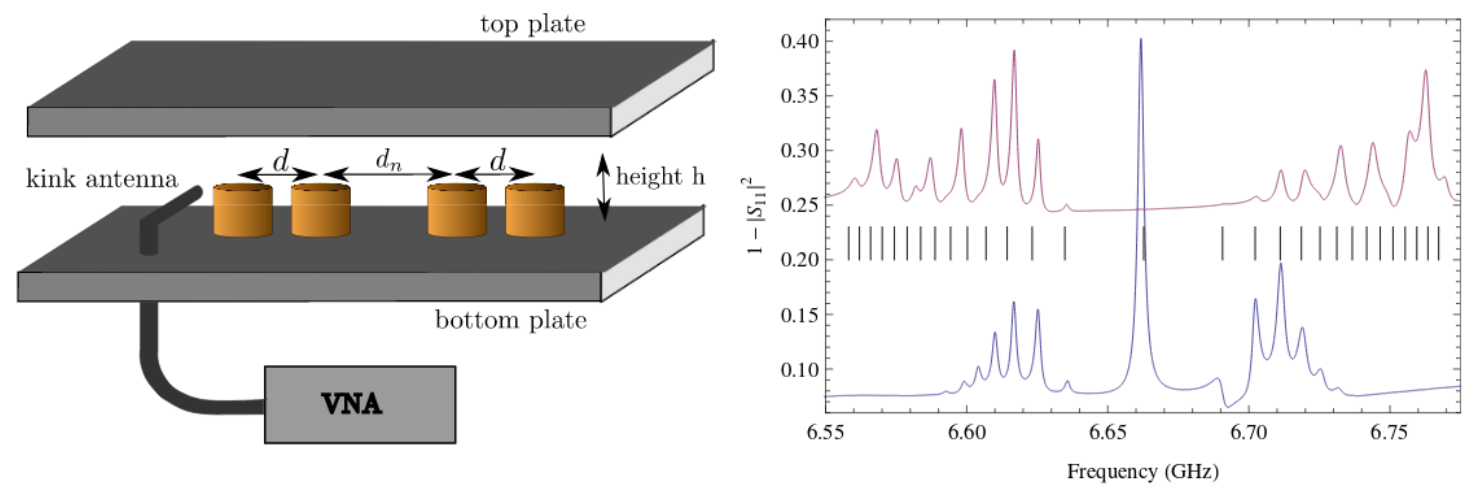

Figura 2.14: A la izquierda se tiene una cadena de discos entre dos placas metálicas, los cuales estan separados a una distancia $d$ o $d_{n}$. A la derecha se muestra el espectro de la reflexión como función de la frecuencia: Las líneas continuas violeta y azul corresponden a medidas experimentales hechas con un VNA en diferentes posiciones de la cadena de dieléctricos. Las líneas verticales negras corresponden a la predicción teórica. [51]

A la izquierda de la figura 2.14 se muestra el montaje experimental compuesto de una cadena de dímeros usado en la referencia [51]. A la derecha se muestra el espectro de frecuencia del sistema de dímeros. La cadena está compuesta de 15 dímeros. Cada dímero está separado entre sí a una distancia de $13 \mathrm{~mm}$. El tamaño de las resonancias depende de la posición de la antena, ya que ésta es proporcional a la función de onda del disco. Para obtener un espectro de frecuencia completo se debe colocar la antena en diferentes posiciones. Las líneas verticales corresponden a las predicciones numéricas hechas con el modelo de tight-binding y hay una excelente coincidencia con el experimento. 

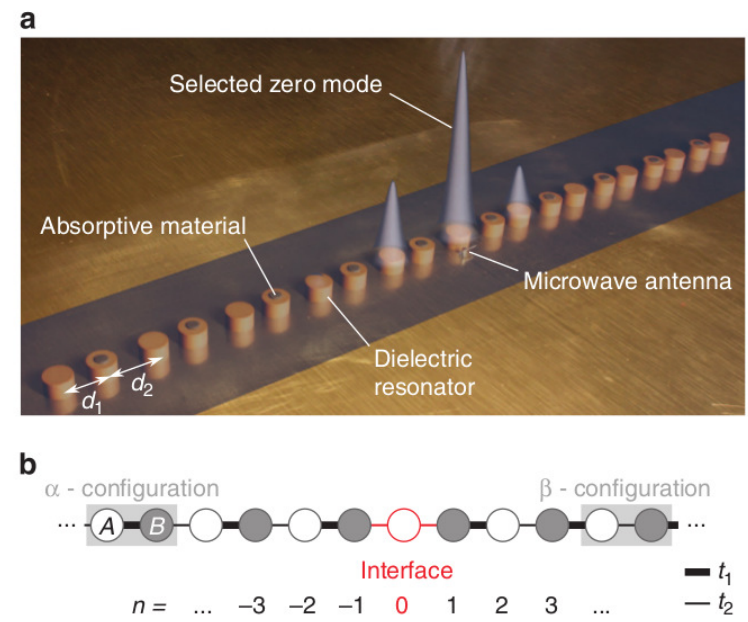

Figura 2.15: Sistema utilizado para la realización experimental de la inducción de un estado, asociado a un defecto, en el espectro de frecuencias de microondas. El sistema se compone de un arreglo unidimensional de dieléctricos acoplados. Cada resonador tiene una dimensión de $5 \mathrm{~mm}$ de altura, 8 de diámetro y un índice de refracción de 6 [52].

En la referencia [52] se plantea otra manera de establecer el modelo de enlace fuerte que es mediante el confinamiento de las ondas electromagnéticas en los resonadores. Estos hacen la función de los átomos en los sitios de la red (ver figura 2.15). La resonancia de un disco dieléctrico aislado es análogo a la energía de sitio de un átomo aislado. Se encontró el acoplamiento en función de la separación entre los resonadores y tomando en cuenta la evanescencia de las ondas electromagnéticas, en dicha referencia, el modelo se desarrolló sólo a primeros vecinos. La ecuación de tight-binding para $n=1,3, \ldots$ del sistema de la figura 2.15 viene dada por

$$
\left(\nu-\nu_{n}\right) \psi_{n}=t_{2} \psi_{n-1}+t_{1} \psi_{n+1},
$$

donde $\mathrm{n}$ es el número de resonandores, $\psi_{n}$ es la amplitud del modo del resonador enésimo, las t son los acoplamientos y finalmente $\nu_{n}$ es la frecuencia de resonancia de un resonador aislado.

\subsection{Modelo de enlace fuerte en elasticidad}

Un ejemplo del uso del método de enlace fuerte en sistemas acústicos fue hecho en la referencia [53]. Los autores describen un sistema cuasiperiódico acústico en 2D usando la aproximación del modelo de tight-binding. El sistema consiste de diapasones acoplados entre sí, usando resortes que conectan los primeros vecinos. Este arreglo de diapasones tiene una geometría cuasicristalina, tipo mosaico de Penrose, como se ve en la figura 2.16. Los diapasones, colocados en el centro de cada rombo, son el análogo acústico-elástico de 
los átomos (átomos artificiales acústicos) y los alambres de acero de $1 \mathrm{~mm}$ de diámetro corresponden a los acoplamientos. La principal característica de estos alambres es que proporcionan un acoplamiento fuerte; es decir, casi no tienen pérdidas. La ecuación que describe al sistema es:

$$
E \Psi(x)=-\sum_{N N}\left[a^{\prime} \Psi\left(x^{\prime}\right)-a \Psi(x)\right],
$$

donde $E$, es la energía del sistema y $\omega^{2}$ es la frecuencia de resonancia acústica del sistema de resonadores. Por su parte $a$, depende de las frecuencias de resonancia de cada diapasón; mientras que $a^{\prime}$, depende de las propiedades del alambre que conecta a dichos diapasones:

$$
a^{\prime}=\omega_{N N}^{2}\left(\frac{\omega l_{N N}}{c} \csc \left(\frac{\omega l_{N N}}{c}\right)\right),
$$

y

$$
a=\frac{\omega_{0}^{2}}{z}+\omega_{N N}^{2}\left(\frac{\omega l_{N N}}{c} \cot \left(\frac{\omega l_{N N}}{c}\right)\right),
$$

$\omega_{0}$ es la frecuencia de resonancia natural de cada diapasón y $z$ es el número de vecinos cercanos. Mientras que $\omega_{N N}=\left(c / l_{N N}\right)\left(m_{N N} / m\right)^{1 / 2}$, con $m$ la masa de los diapasones, $l_{N N}$ la longitud de los alambres y $m_{N N}$ la masa de los alambres. En dicho trabajo; sin embargo, las limitaciones experimentales no permitieron hacer una comparación confiable de los resultados experimentales con la simulación del modelo.

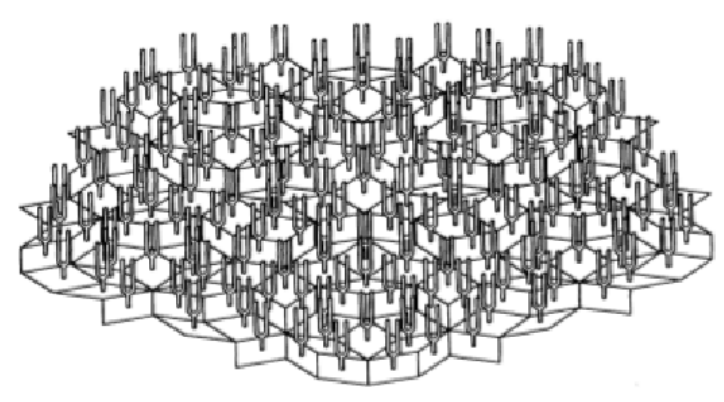

Figura 2.16: Sistema de diapasones acoplados en red tipo mosaico de Penrose [53].

La ecuación característica del modelo de enlace fuerte para distintos sistemas se da en la tabla 2.1. Se hace una comparación de los parámetros físicos que involucran para cada uno de los modelos expuestos en este capítulo. 


\begin{tabular}{|l|c|c|c|c|}
\hline Modelo & Ecuación & Variables & Sistema aislado & Acoplamiento \\
\hline Cuántico & $C_{n-1} V+C_{n} \epsilon_{n}+C_{n+1} V=E C_{n}$ & $\begin{array}{c}\text { Energía, función } \\
\text { de onda }\end{array}$ & $\begin{array}{c}\text { energía } \\
\text { de sitio }\end{array}$ & $\mathrm{V}$ \\
\hline Mecánico & {$\left[2 u_{n}-u_{n+1}-u_{n-1}\right]=\omega^{2} u_{n}$} & $\begin{array}{c}\text { Frecuencia, desplazamiento } \\
\text { de la posición de equilibrio }\end{array}$ & $\begin{array}{c}\frac{2 C}{m}, \text { frecuencia } \\
\text { de resonancia }\end{array}$ & $\frac{C}{m}$ \\
\hline Microondas & $t \Psi_{n-1}+t \Psi_{n+1}+\nu_{n} \Psi_{n}=\nu \Psi_{n}$ & Frecuencia, campo & $\begin{array}{c}\text { frecuencia } \\
\text { de un disco }\end{array}$ & $\begin{array}{c}\text { distancia } \\
\text { entre discos }\end{array}$ \\
\hline Elástico & $A_{n-1} C+A_{n} f_{0}+A_{n+1} C=f A_{n}$ & $\begin{array}{c}\text { Frecuencia, amplitud de } \\
\text { vibración en el defecto }\end{array}$ & $\begin{array}{c}\text { frecuencia } \\
\text { de un defecto }\end{array}$ & $\begin{array}{c}\text { C distancia } \\
\text { entre defectos }\end{array}$ \\
\hline
\end{tabular}

Tabla 2.1: Modelo de enlace fuerte adaptado a diversos sistemas. 


\section{Capítulo 3}

\section{Diseño de un cristal elástico artificial usando matriz de transferencia}

En este capítulo se analiza el efecto que tienen, en el espectro de frecuencias asociado a un sistema localmente periódico, los distintos parámetros físicos manipulables para diseñar la estructura de un cristal elástico sobre una varilla uniforme como son: la profundidad, anchura de las muescas, sección transversal, longitud de una celda unitaria simple, un defecto, dos defectos y varios defectos, entre otros. En la sección 3.1 se describe la ecuación de onda para las vibraciones torsionales en una varilla con sección transversal cuadrada. En la sección 3.2 se estudia cada uno de los parámetros que componen un sistema localmente periódico. En la sección 3.3, se inicia el diseño numérico de la supercelda. En la sección 3.4 se analiza el decaimiento de la amplitud de onda torsional asociada a un sistema con un defecto. Para la sección 3.5, se hace el mismo análisis de la sección 3.4 esta vez para dos defectos acoplados y por último en 3.6 se analizan las estructuras localmente periódicas con 3 hasta 6 defectos, que más tarde serán comparadas con las predicciones del modelo teórico de acoplamiento o mejor conocido como tight-binding.

\subsection{Vibraciones torsionales en una varilla simple}

La ecuación de onda 3.1, que describe las vibraciones torsionales de una varilla como la que se muestra en la figura 3.1 es: 


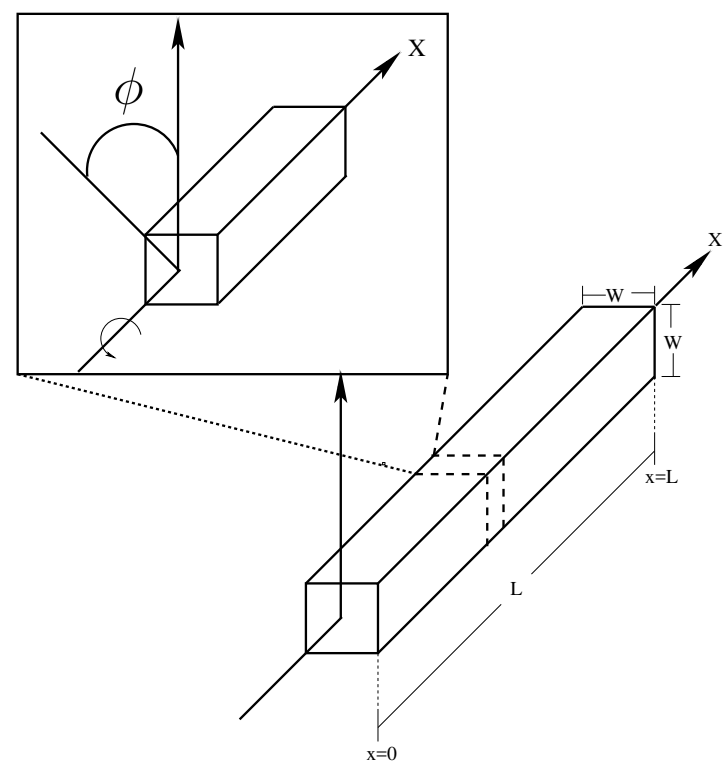

Figura 3.1: Perspectiva 3D de una varilla elástica de sección transversal cuadrada, de área $W^{2}$ y longitud L. En el recuadro se muestra la amplificación de un elemento de la varilla, donde se indica el ángulo de torsión $\phi$ alrededor del eje $x$ que sufre la varilla al paso de una onda torsional.

$$
\frac{\partial^{2} \phi}{\partial x^{2}}=\frac{1}{c^{2}} \frac{\partial^{2} \phi}{\partial t^{2}}
$$

donde $\phi(x, t)$ es el ángulo de torsión a lo largo del eje de simetría $x$ de la varilla; es decir, depende de la posición, $x$, y del tiempo $t$. La velocidad de estas ondas, en la varilla, es c y está relacionada con la sección transversal de la varilla y el material del cual está fabricado, como sigue:

$$
c=\sqrt{\frac{G \eta}{\rho I}},
$$

donde, $\rho$, es la densidad de masa del material; $G$, es el módulo de corte; $I$, es el momento de inercia polar [54], y $\eta=1$ para una varilla simple.

La ecuación (3.1) se puede resolver por separación de variables, introduciendo la constante, $-\omega^{2}$, de manera que; si

$$
\phi(x, t)=\psi(x) e^{-i \omega t}
$$

entonces,

$$
\psi(x)=a e^{i k x}+b e^{-i k x} ; \quad k=\frac{\omega}{c} .
$$

Tomando encuenta las condiciones de frontera libre en ambos extremos (de la varilla de la figura 3.1), se considera: 


$$
\left.M_{T}(x, T)\right|_{x=0}=\left.M_{T}(x, T)\right|_{x=L}=0,
$$

donde $M_{T}$, es el momento de torsión alrededor de $x$ definido por:

$$
M_{T}=G \alpha \frac{\partial \phi(x, t)}{\partial x} .
$$

Con $G$ el módulo de corte. A partir de la ecuación (3.4) y usando las condiciones de frontera (3.5), es posible mostrar que las soluciones estacionarias de la ecuación 3.3 son:

$$
\psi_{n}(x)=2 a \cos \left(k_{n} x\right), \quad \text { donde } k_{n}=\frac{n \pi}{L}
$$

y $a$ es la amplitud.

\subsection{Sistema elástico localmente periódico}

En la figura 3.2 se muestra un sistema elástico localmente periódico cuasi 1D, cuyo comportamiento del espectro de frecuencia de vibraciones torsionales asociado, puede estudiarse numéricamente como función de sus distintos parámetros físicos característicos.

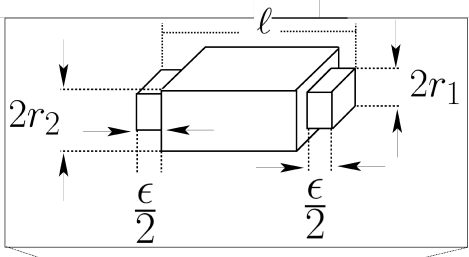

Figura 3.2: Sistema localmente periódico compuesto por 15 celdas unitarias simples, maquinadas sobre una varilla de aluminio 6061-T6 de seción transversal cuadrada. En la parte superior se muestra una ampificación de la celda unitaria simple, que caracteriza a esta estructura periódica. Los parámetros relevantes de la estructura y sus dimensiones se indican con las flechas, $r_{1}, r_{2}, \epsilon, \ell$, etc . En dicha celda simple se indican los parámetros como profundidad $r_{2}-r_{1}$ y con $\epsilon / 2$ se indica la mitad de la muesca.

La celda simple unitaria se muestra en el recuadro donde se indican todas sus dimensiones y parámetros relevantes. La longitud total de la celda unitaria es $\ell ; \epsilon$ es el ancho de una muesca o ranura entre dos bloques; $2 r_{2}$ es la longitud de un lado de la sección transversal cuadrada, de la varilla original; $2 r_{1}$ es la longitud de un lado de la sección tranversal cuadrada cuando esta ha sido ranurada en cada uno de sus 4 costados. Así $r_{2}-r_{1}$ es la profundidad de cada muesca maquinada en el sistema elástico. El sistema estructurado de la figura 3.2 consta de 15 celdas unitarias simples, (como las del recuadro 
acopladas entre sí) y será el primer sistema que simularemos numéricamente, usando el método de la matriz de transferencia (MMT), que se explica en el apéndice A.

Un sistema similar a este ha sido reportado por Gutiérrez et al, [6] donde estudian las vibraciones torsionales de una varilla de aluminio, con sección transversal circular con estructura periódica equivalente. Este sistema, es el sistema más simple que podemos proponer, como punto de partida de una estructura elástica localmente cristalina.

A continuación, se explora numéricamente cada uno de los parámetros relevantes de la celda unitaria mostrada en la figura 3.2 que ya mencionamos con anterioridad y como afectan la estructura de bandas del correspondiente espectro de frecuencias torsionales. Esto es con la finalidad de poder diseñar un sistema que permita distinguir parte de su estructura de bandas en un intervalo de (1 a 40) $\mathrm{kHz}$ debido a que los instrumentos para la caracterización por espectroscopia acústica resonante (ARS) sólo nos permite medir confiablemente en ese intervalo. En particular el amplificador del cual disponemos es un CERWIN VEGA que trabaja en el rango audible, el analizador de redes trabaja de $3 \mathrm{~Hz}$ a $300 \mathrm{MHz}$ y los EMATs trabajan de $100 \mathrm{~Hz}$ a $60 \mathrm{kHz}$.

\subsubsection{Determinación del parámetro $\eta$}

El primer párametro que vamos a explorar es $\eta$, el cual se define como: $\eta=\frac{r_{1}}{r_{2}}$. Este parámetro esta asociado a la profundidad de las muescas que acoplan los bloques del sistema periódico y toma valores entre 0 y 1 . Donde $\eta=1$ corresponde a una varilla sin estructura lisa, simple; es decir, no hay muescas maquinadas sobre ella; mientras que, $\eta=0$, corresponde al caso límite en el que el conjunto de celdas unitarias se desacoplan o separan completamente; es decir, la ranura fue tan profunda que separo los bloques de la estructura originalmente periódica.

Debido a que la simulación numérica, basada en MMT, solo requiere los parámetros que determinan el perfil longitudinal del sistema elástico de interés, de aquí en adelante solo mostraremos los perfiles o cortes longitudinales del sistema mostrado en la figura 3.2 o similares, como los que se muestran en la figura 3.3a. 


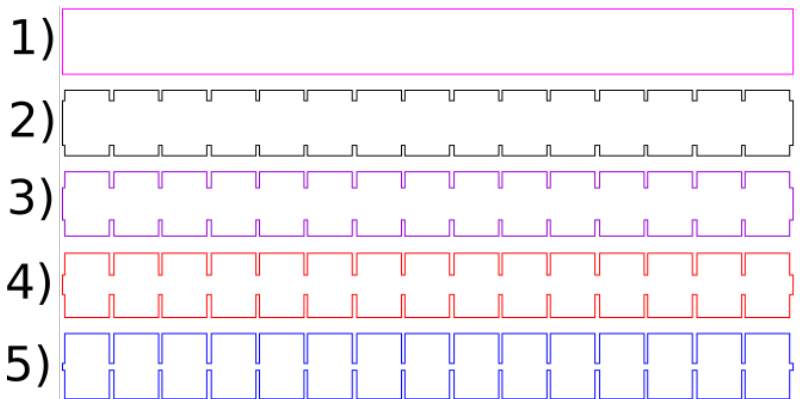

(a)

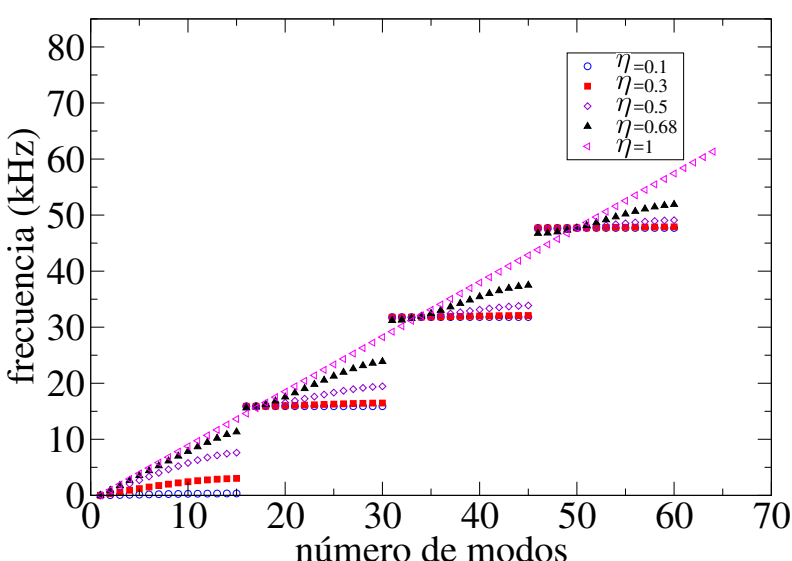

(b)

Figura 3.3: (a) Corte longitudinal del sistema localmente periódico, que corresponde en orden descente a los valores $\eta=1, \eta=0.68, \eta=0.50, \eta=0.30$ y a $\eta=0.10$. (b)Espectros de frecuencia torsional correspondientes a cada uno de los sistemas de la izquierda.

En la figura 3.3a se muestra un corte longitudinal del sistema de la figura 3.2 para 5 diferentes valores de $\eta$. En la misma figura se muestra la gráfica del espectro de frecuencias torsional como función del número de modos, para cada valor de $\eta$. Los símbolos en triángulos izquierdo, que definen una línea recta, corresponden a la varilla de arriba, asociada al valor de $\eta=1$; mientras que las 4 estructuras de bandas de la gráfica (triángulos arriba, diamante, cuadros y círculos) corresponden al sistema periódico con valores de $\eta=0.68, \eta=0.5, \eta=0.3$ y $\eta=0.1$, respectivamente. El espectro para cada uno de estos valores de $\eta$ ya muestra una estructura de bandas y brechas características de sistemas periódicos. En particular, existen dos espectros muy similares, correspondientes a $\eta=0.3$ y $\eta=0.1$. Estos sistemas presentan una ranura muy profunda, lo cual es poco práctico para la fabricación de los mismos, por lo cual fueron descartados. Asimismo, existen otras dos estructuras $(\eta=0.5$ y $\eta=0.68)$ cuyos espectros ya presentan diferencias notables en sus bandas y brechas. Elegimos para el diseño el parámtero $\eta=0.68$, porque facilita la fabricación de un sistema poco frágil para su manipulación en el experimento, además de que ya muestra compartamiento característico de sistema periódico.

En la figura 3.3b se observa que a diferencia del espectro de un cristal atómico (ver figura 2.9), los niveles de las bandas emergentes no se distribuyen alrededor de las frecuencias de las resonancias del espectro, correspondiente a una única celda sino que se despliegan en forma creciente a partir de la frecuencia original. Es por esto que la celda unitaria de este sistema no nos sirve para emular, con un sistema elástico, a un cristal atómico, que es nuestro objetivo principal.

De aquí en adelante para todos los posteriores diseños, el valor del parámetro $\eta=0.68$ quedará fijo. Note que para ese valor de $\eta$ se distinguen dos bandas y dos brechas, dentro del rango; es decir, de 1 a $30 \mathrm{kHz}$. 


\subsubsection{Determinación del valor del parámetro $\epsilon$}

El segundo parámetro por explorar, numéricamente, es la anchura de la muesca maquinada, $\epsilon$, manteniendo la profundidad constante, $\eta=0.68$, determinada en la sección anterior. En la figura 3.4a se muestran los perfiles del sistema elástico periódico correspondiente a 3 valores distintos del parámetro $\epsilon$ y profundidad $\eta=0.68$. El sistema 1) tiene una anchura de la muesca de $\epsilon=4 \mathrm{~mm}$; el 2), de $\epsilon=8 \mathrm{~mm}$ y el 3) de $\epsilon=12 \mathrm{~mm}$.

Este sistema periódico de celdas simples no tiene las propiedades espectrales similares a las de un cristal atómico debido a la distribución de sus niveles como se discutió en la sección anterior. Es necesario diseñar un sistema elástico periódico, cuya celda unitaria (más compleja) permita imitar al cristal atómico en sus propiedades espectrales (como en la referencia [16]). Las brechas del espectro torsional más anchas (lo cual es una ventaja porque ahí tendremos niveles aislados) corresponden al caso de $\epsilon=8 \mathrm{~mm}$ y $\epsilon=12 \mathrm{~mm}$. El ancho de la muesca del sistema 3) es mayor que 2), lo cual es más complejo de construir porque no existen cortadores tan grandes, debido a eso elegimos el valor de $\epsilon=8 \mathrm{~mm}$.

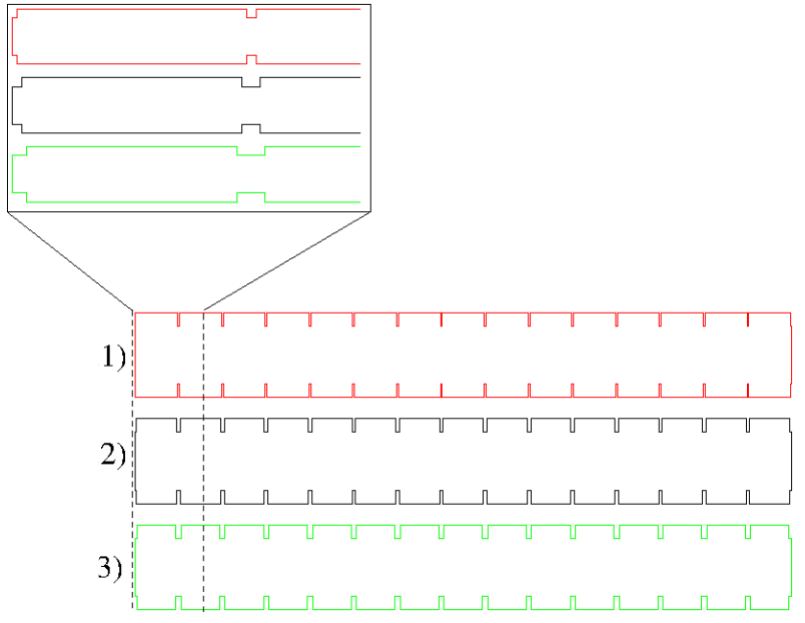

(a)

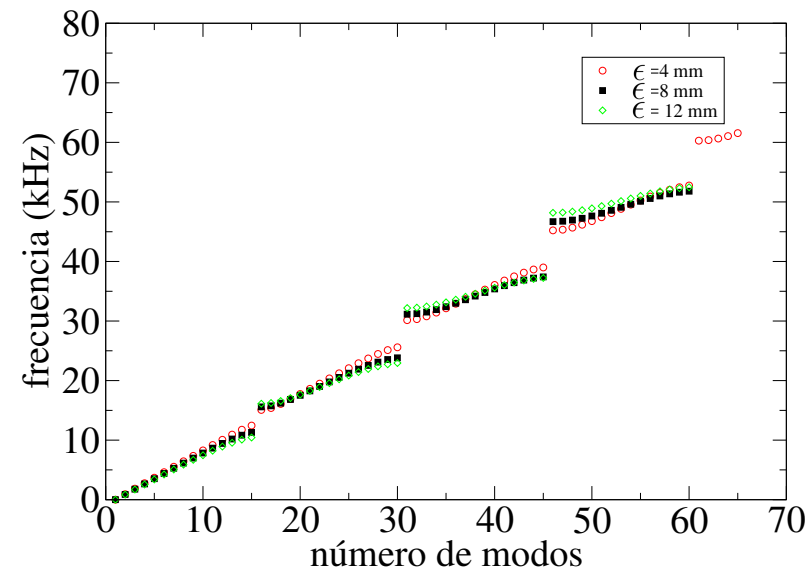

(b)

Figura 3.4: Estructuras periódicas elásticas: (a) se muestran 3 sistemas, localmente periódicos correspondientes (en orden descendente) a los valores de a.1) $\epsilon=4 \mathrm{~mm}$, a.2) $\epsilon=8$ $\mathrm{mm}$ y a.3) $\epsilon=12 \mathrm{~mm}$. En el recuadro superior izquierdo se muestra que la diferencia entre los sistemas es el ancho de la muesca. (b) Espectros de frecuencias torsionales correspondientes a cada uno de los sistemas elásticos periódicos.

En la gráfica 3.4b se muestran los espectros de frecuencias torsionales asociados a los sistemas de la figura 3.4a. Debido a que los 3 sistemas tienen el mismo número de celdas unitarias, esto se verá reflejado en el mismo número de niveles. Además el espectro está compuesto por 4 gaps, siendo el segundo el de mayor anchura. Esto será de gran ayuda para el diseño final. Hasta ahora, hemos fijado el valor de dos parámetros relevantes, para el diseño elástico periódico de celdas simples $\eta=0.68$ y $\epsilon=8 \mathrm{~mm}$. Debemos de tener presentes estos valores para el resto del diseño de la nueva celda unitaria compleja. 


\subsubsection{Determinación de la sección transversal del sistema}

Anteriormente se mencionó que existen estudios de sistemas periódicos de celdas simples en varillas con sección transversal circular como se muestra en la referencia [6]. En este estudio simulamos las vibraciones de dicho sistema y las comparamos con las de un sistema equivalente de sección transversal cuadrada y rectangular como las venden los fabricantes de varillas de aluminio. La forma de la sección transversal del sistema, es un parámetro geométrico asociado, a la velocidad de propagación de las ondas torsionales en cada varilla, $c$. Se puede mostrar que las velocidades de propagación de estas ondas, en varillas de aluminio de sección transversal cuadrada, circular y rectangular son $c=2852 \mathrm{~m} / \mathrm{s}, c=3100 \mathrm{~m} / \mathrm{s}$ y $c=2294 \mathrm{~m} / \mathrm{s}$, respectivamente ref. [55]. En este estudio, se consideraron secciones transversales: cuadrada, circular y rectangular. Los espectros numéricos correspondientes se muestran en la figura 3.5.



Figura 3.5: Comparación de espectros de frecuencias torsionales, para varillas periódicas de celda unitaria simple, con: sección transversal circular, cuadrada, rectangular respectivamente y con un $\eta=0.68$ y un $\epsilon=8 \mathrm{~mm}$.

En la gráfica de la figura 3.5 se puede observar que las bandas asociadas al sistema de sección transversal circular se encuentran en intervalos de frecuencia más altos que para los otros dos sistemas (los de sección transversal cuadrada y rectangular). Esto dificulta las medidas experimentales ya que podríamos salir del rango audible que es el que nos permite nuestro montaje experimental. Adicionalmente, es más difícil fabricar un sistema con sección transversal circular, por lo tanto descartamos este modelo de varilla con sección transversal circular para nuestro diseño. Por otro lado, a pesar de que el modelo de sección transversal rectangular muestra sus bandas permitidas a frecuencias más bajas que para el caso de la varilla de la sección transversal cuadrada, los niveles de frecuencias en cada banda se encuentran menos espaciados, este hecho dificulta las mediciones de los niveles en cada banda. Por este motivo elegimos para continuar nuestro diseño varillas con sección transversal cuadrada. 


\subsection{Sistema elástico localmente periódico diseñado}

Con los parámetros físicos determinados en las secciones anteriores: $\eta=0.68, \epsilon=8 \mathrm{~mm}$ y varilla de sección transversal cuadrada de lado $1 / 4^{\prime \prime}$, hemos determinado la celda unitaria simple con la que construimos un sistema elásico localmente periódico. Este sistema será modificado para diseñar una celda unitaria compleja que nos permita construir un cristal elástico con propiedades espectrales específicas similares a las de un sistema cuántico.



Figura 3.6: Abajo: Perfil del sistema elástico, localmente periódico, de 15 celdas unitarias simples. Arriba, se muestra la imagen amplificada del perfil de la celda unitaria simple, donde se indican los parámatros relevantes de la misma.

En la figura 3.6 se muestra el perfil longitudinal elástico que consta de 15 celdas simples de la sección anterior las cuales fueron numeradas. También se indican sus parámetros característicos. El espectro numérico, de frecuencia torsionales obtenido con MMT se muestra en la figura 3.7. 


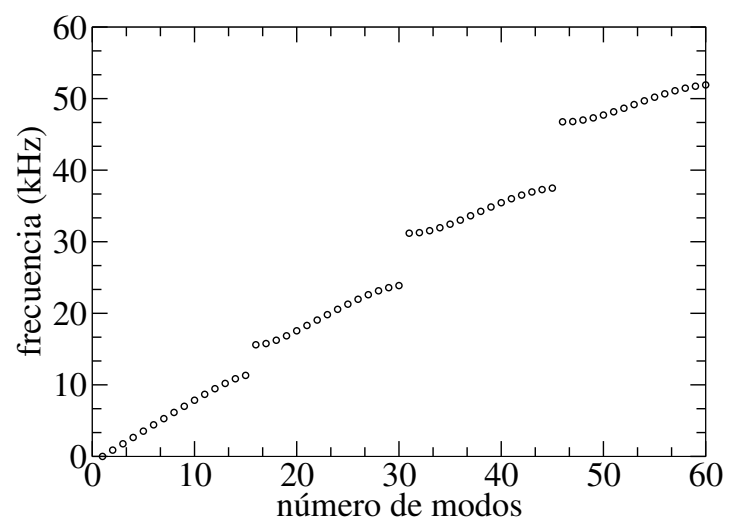

Figura 3.7: Espectro de frecuencias torsional, como función del número de modos del sistema de la figura 3.6. En el intervalo de 0 a $60 \mathrm{kHz}$ se observan 4 bandas y 3 brechas. Cada banda se conforma de 15 niveles de frecuencia.

En el intervalo de frecuencias de 0 a $60 \mathrm{kHz}$ se observan 4 bandas permitidas y 3 brechas. Cada banda contiene 15 niveles correspondientes, asociados a las vibraciones torsionales de las 15 celdas unitarias acopladas. En este intervalo se muestran, en total, 45 frecuencias de vibración torsional, propias del sistema de la figura 3.6.

En la figura 3.8 se muestran las amplitudes de onda torsionales propias, asociadas a los primeros 45 frecuencias de modo normal que aparecen en el espectro de arriba. En cada recuadro se grafica la amplitud de onda torsional como función de la posición del sistema periódico de la figura 3.6. Los modos normales asociados a frecuencias más altas fluctúan más, como es de esperarse (ver últimos recuadros de la figura 3.8). Todos estos patrones estacionarios son funciones extendidas a lo largo del sistema, por lo que no son buenos candidatos para formar una base que genere un modelo de enlace fuerte (ver sección 2). 


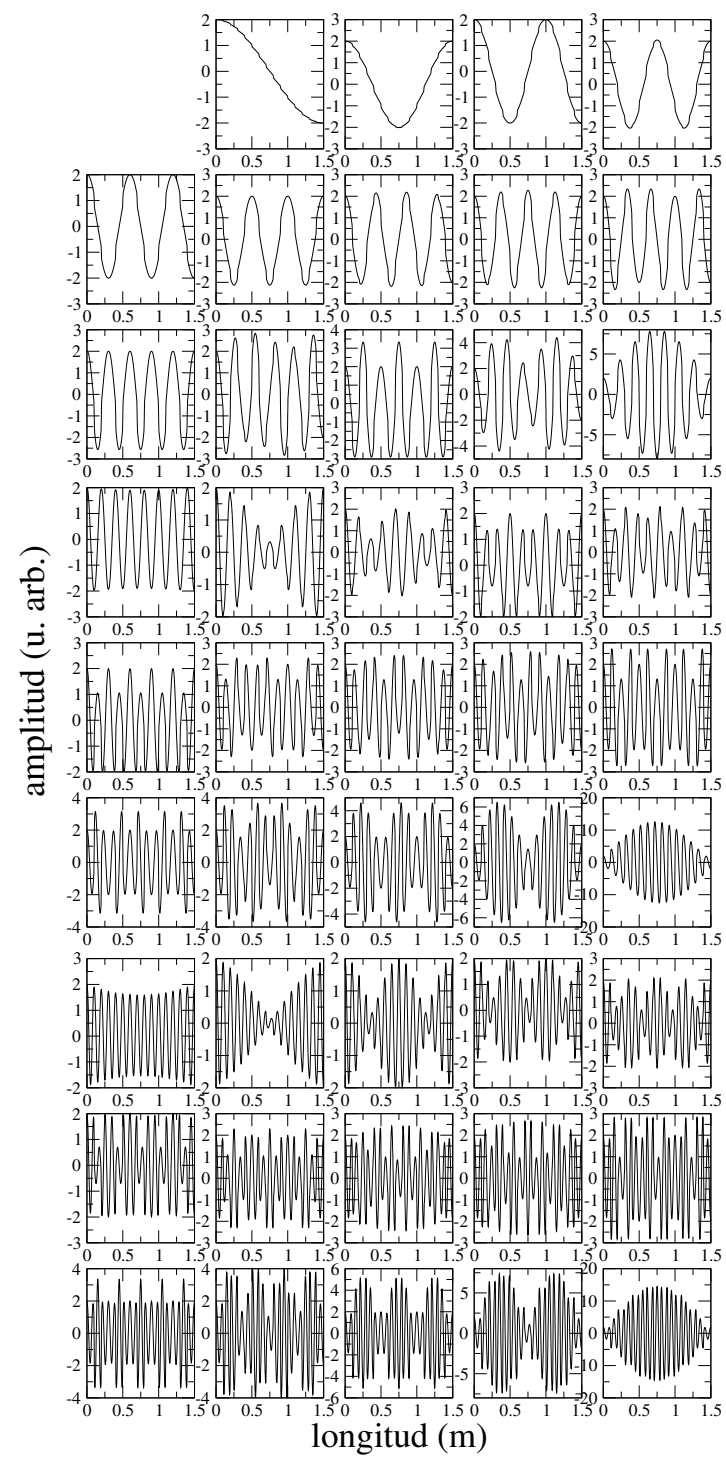

Figura 3.8: Primeros 45 modos normales torsionales del sistema elástico, localmente periódico, de la figura $3.6(\mathrm{n}=0$ al $\mathrm{n}=44)$ asociados a las primeras tres bandas permitidas

Similar al sistema de la referencia [6], este sistema tampoco nos sirve para construir un modelo de enlace fuerte porque ninguna de las amplitudes de onda estacionarias, están localizadas. Esto quiere decir, que en vez de que la amplitud de onda concentre su máxima amplitud en zonas pequeñas y decaiga rápidamente a los alrededores, ésta se distribuye equitativamente a lo largo de toda la barra o del sistema (ver figura 3.8). 


\subsection{Sistema localmente periódico con un defecto}

Como primera modificación al sistema originalmente periódico, de celda unitaria simple, se introduce un defecto en la estructura y se observan sus efectos en el espectro torsional. Para introducir un defecto en el sistema, se modifica una celda unitaria simple, cambiando en ancho de la costilla de forma simétrica (ver figura 3.9) respecto al centro de la celda simple. A este tipo de defecto se le conoce como defecto sustitucional simétrico. El defecto se realizó sobre la celda simple No. 6 del sistema acoplado de 15 celdas simples (ver figura 3.10) para evitar o reducir los efectos de frontera (orilla) en el sistema.

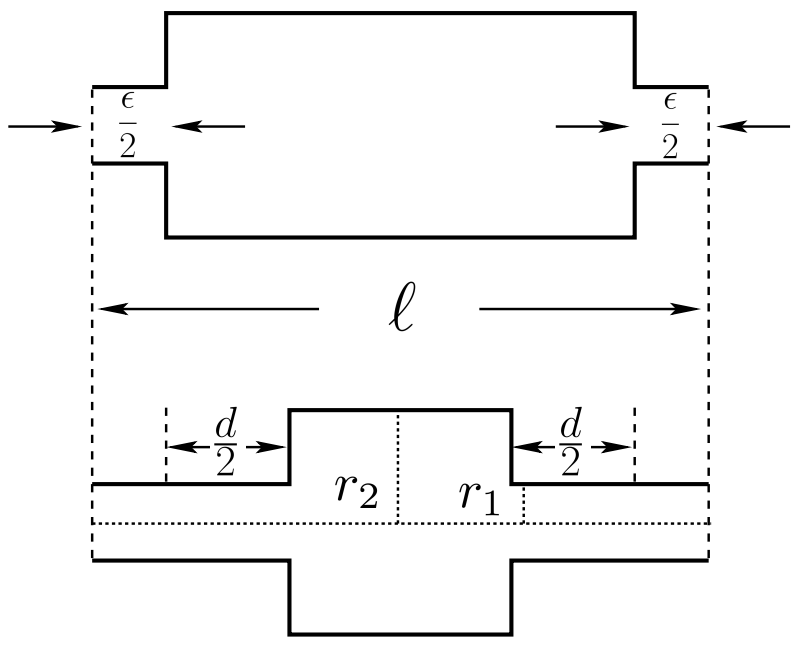

Figura 3.9: Arriba: Celda simple; abajo: Celda simple modificada. $\epsilon$ : es el ancho de la muesca, d: es la parte ranurada.

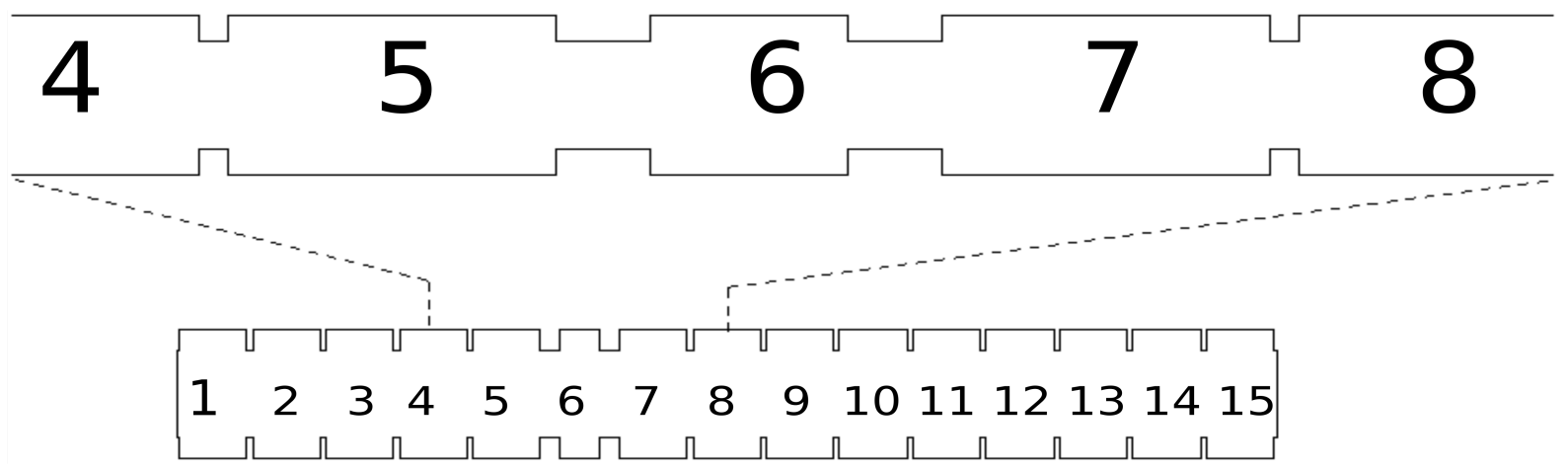

Figura 3.10: Estructura elástica de 15 celdas unitarias con un defecto en la celda No. 6. Abajo: número de celdas $\mathrm{nc}=15$, longitud $\mathrm{L}=1.5 \mathrm{~m}$, longitud en el sitio 6 (ancho del defecto) $=9.2 \mathrm{~cm}$, ancho de la muesca $\epsilon=8 \mathrm{~mm}, \eta=0.68$; arriba: sistema con un defecto (zoom) 


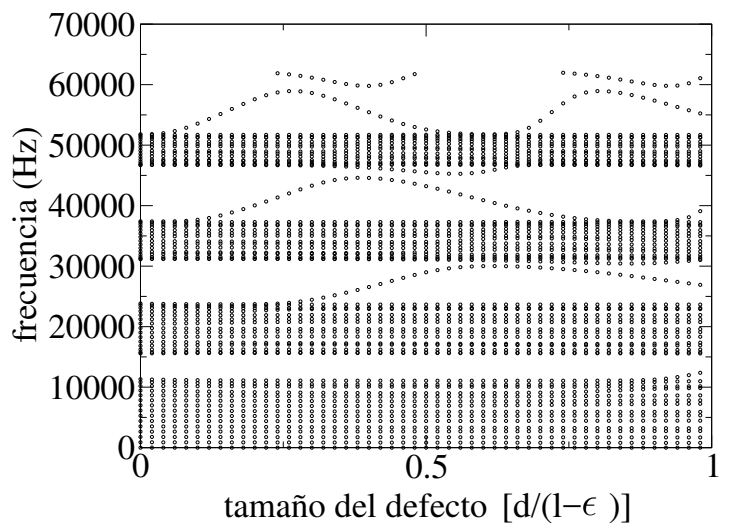

Figura 3.11: Espectro de frecuencia torsional en función del tamaño del defecto.

Para este intervalo de frecuencias el espectro del sistema de la figura 3.10 muestra, en la segunda y en la tercera brecha, el inicio de una nueva banda emergente, de frecuencias propias, asociadas al defecto. La posición de dicho nivel nos favorece porque la frecuencia a la que se encuentra está dentro del intervalo de frecuencia del audible; lo cual es indispensable para poder ser medido; es por tal motivo que estudiaremos sólo el primer nivel en el segundo gap.

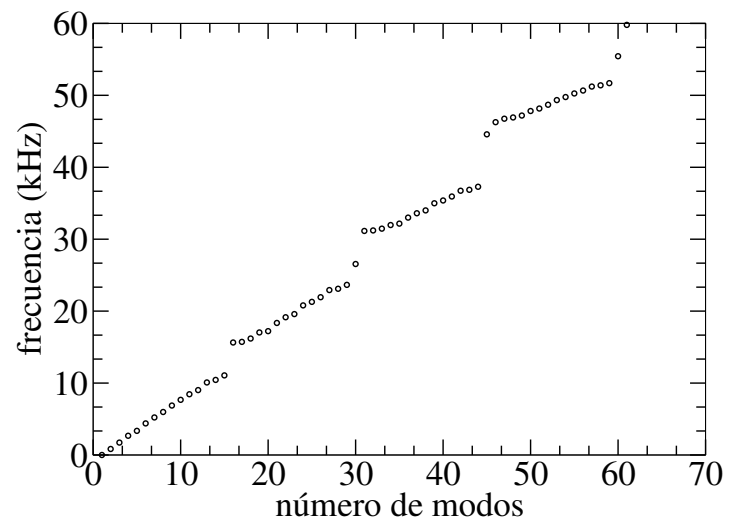

Figura 3.12: Espectro de un sistema periódico de 15 celdas simples nc=15, con un defecto, $\mathrm{L}=1.5 \mathrm{~m}, \epsilon=8 \mathrm{~mm}, \eta=0.68$. Se observa que un nivel se ha movido en el segundo, tercero y cuarto gap del sistema original.

En la figura 3.13 se observan las amplitudes de onda asociadas a los primeros 30 modos normales del sistema que corresponden a sus primeras dos bandas. 


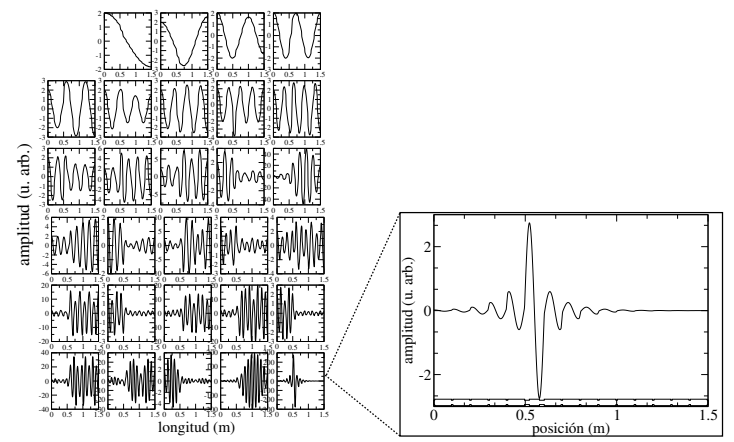

Figura 3.13: Modo del 0-29 de una varilla periódica con un defecto

En la amplificación de la gráfica 3.13 se aprecia como el decaimiento de la amplitud de onda se distribuye hacia ambos lados del defecto de manera simétrica. Esta amplitud está localizada a diferencia de las amplitudes que se encuentran en la banda, las cuales estan extendidas. Esta propiedad de localización es debido a la brecha y se manifiesta por un decaimiento exponencial que rápidamente decae a cero.

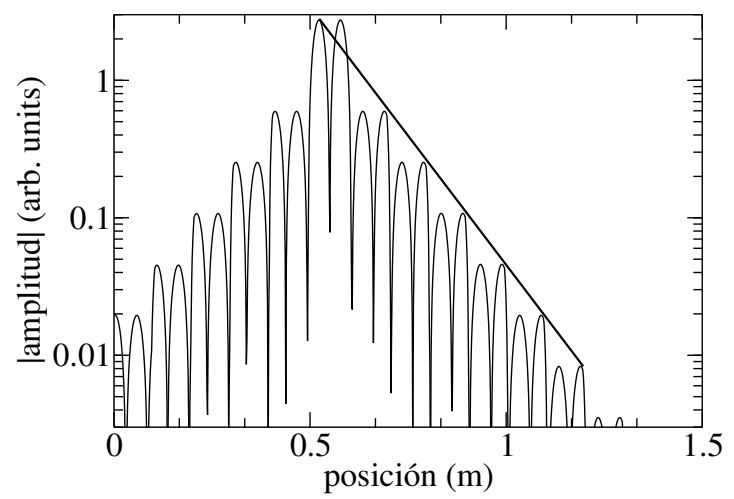

Figura 3.14: Amplitud de onda como función de la longitud del sistema periódico con un defecto, correspondiente al modo $n=30$ que aparece en el 2do gap.

En la gráfica 3.14 se grafica el valor absoluto de la amplitud de onda torsional en escala semilogarítmica. Este patrón está localizado alrededor del modo normal que corresponde a $n=30$, del sistema 3.10. Asimismo este perfil corresponde a la frecuencia en el gap. Note que la gráfica muestra un decaimiento exponencial que es lo quería lograr para generar un modelo TB. Es por esto que solo queda por definir el número de bloques de la supercelda.

A lo largo de este trabajo se hicieron exhaustivas simulaciones numéricas, usando el método de la matriz de transferencia (TMM), para diseñar la celda unitaria adecuada, para construir la estructura elástica, localmente periódica, cuyo espectro de frecuencia muestre una fenomenología análoga a la de un cristal atómico, que además pueda ser medido por los equipos experimentales de que disponemos $(20 \mathrm{~Hz}$ a $20 \mathrm{kHz})$. A continuación se presenta la celda unitaria compleja que fue diseñada (ver figura 3.15).

En la figura 3.15 se muestra, esquemáticamente la geometría de la supercelda unidimensional propuesta para construir el cristal artificial elástico del presente estudio, para 
ondas torsionales. Esta figura consiste de un arreglo de segmentos con sección transversal cuadrada, cuyo número óptimo de segmentos y dimensiones de estas se determinaron numéricamente. Esta supercelda será maquinada sobre una varilla de aluminio 6061-T6 de sección transversal cuadrada, cuyo grosor original es 1/2" (como las vende el fabricante). La estructura de dicha supercelda se logró variando el grosor de la varilla como función de su longitud, según el diseño. Así, la nueva celda unitaria compleja consta de 5 bloques, que mantienen la sección transversal original, unidos por 4 segmentos de longitud $8 \mathrm{~mm}$ obtenidos a partir de la ranuración de la varilla a una cierta profundidad. En las simulaciones numéricas también fueron consideradas las propiedades del material 6061-T6, cuya velocidad de ondas torsionales es de $c=2852 \mathrm{~m} / \mathrm{s}$. Es importante notar que la supercelda unitaria elegida para fabricar el sistema no es única, para obtener las características deseadas, sin embargo por cuestiones prácticas de fabricación se eligió así. Así, se variaron diversos parámetros para determinar las dimensiones de la celda unitaria en una varilla de aluminio y se llegó a establecer una celda unitaria compuesta como la que se muestra en la figura 3.15. La cual la llamaremos de aquí en adelante supercelda.

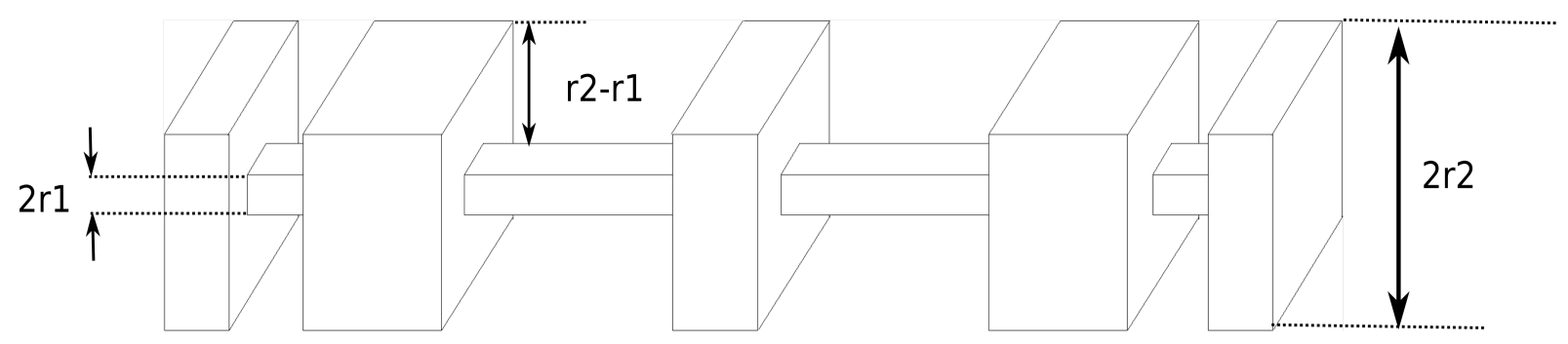

Figura 3.15: Celda unitaria compleja compuesta por 5 costillas y 4 muescas ó supercelda diseñada para la construcción de un cristal artifical elástico.

\subsection{Sistema localmente periódico con dos defectos}

En la figura 3.16b se muestra el espectro de frecuencia para ondas torsionales correspondiente a un sistema de 2 defectos en términos de los parámetros $l, \epsilon \mathrm{y} d$. De la figura 3.9 definimos el parámetro $h=\frac{d}{l-\epsilon}$; cuando $h=0$ el sistema es periódico con celda simple y su espectro tiene una estructura de bandas y brechas. Conforme se deforma un defecto de la celda simple, $h \neq 0$ la estructura de bandas del sistema periódico de la figura 3.9 inciso a) parte superior, sufre modificaciones. En particular, para $h=0.4$, existen 2 niveles, asociados al bloque deformado, que se introducen en la segunda brecha. Los sistemas diseñados consideran una deformación $h=0.4$. En el caso límite de $h=1$ el bloque deformado tiene anchura 0, que es la máxima deformación que podemos generar. 


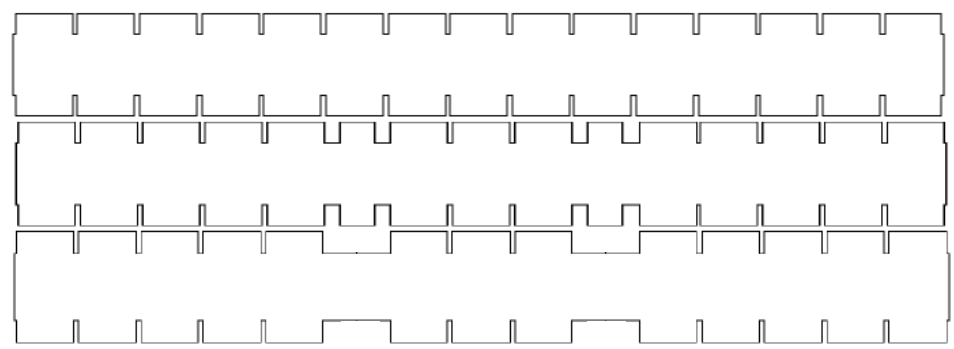

(a)

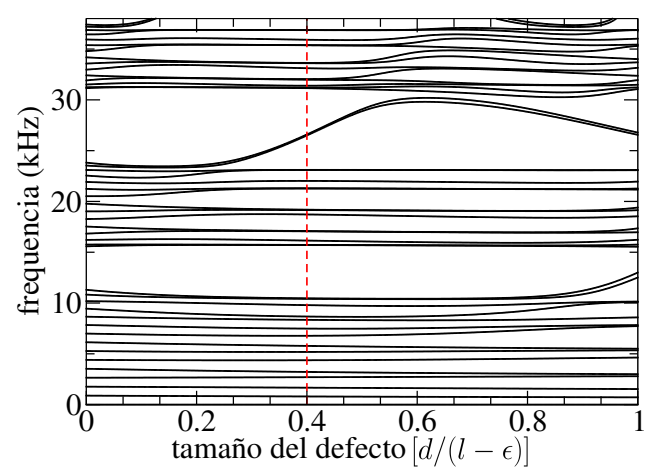

(b)

Figura 3.16: Casos límites del sistema elástico estructurado de 2 superceldas acopladas: (a) Se muestra el corte longitudinal de 3 sistemas estructurados, arriba $h=0$, el sistema es periódico; en medio hay 2 defectos $h=0.4$ insertados en el sistema periódico; abajo $h=1$. b) Se muestra el espectro de frecuencias numérico como función de h para el diseño de la super-celda, como función de uno de los parámetros del sistema, $h$ o tamaño de la costilla para un sistema periódico de 15 celdas con 2 defectos.

En la gráfica de la figura 3.16b se muestra el espectro de frecuencia de ondas torsionales numérico de 2 super-celdas acopladas como función del parámetro h. Podemos observar como algunos niveles del espectro del sistema elástico aparecen en la 1ra y la 2da brecha debido a la deformación de 2 bloques. Estos niveles en tales brechas indican el inicio del surgimiento de nuevas bandas de frecuencia del sistema. En la figura 3.17a se muestra el espectro alrededor de la segunda brecha, tales niveles se distribuyen alrededor del correspondiente nivel de frecuencia del sistema de una celda elástica aislada. Las dos gráficas (3.17b y 3.17c), muestran las amplitudes de onda asociadas a cada uno de los dos niveles de frecuencia emergentes en el 2do gap. Ambas amplitudes de onda están localizadas al centro de cada uno de los dos defectos. 


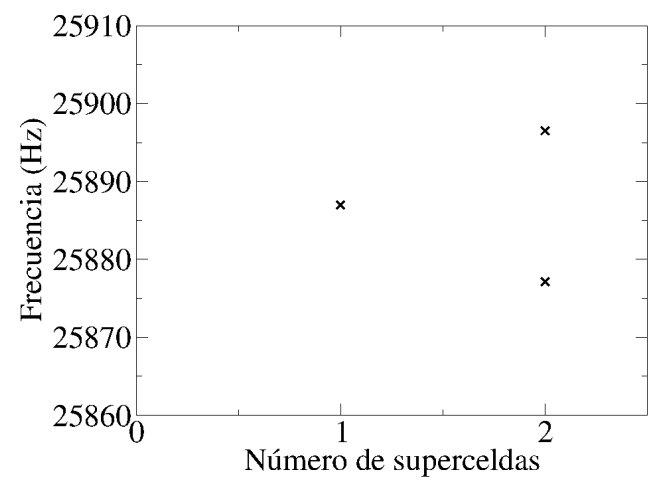

(a)

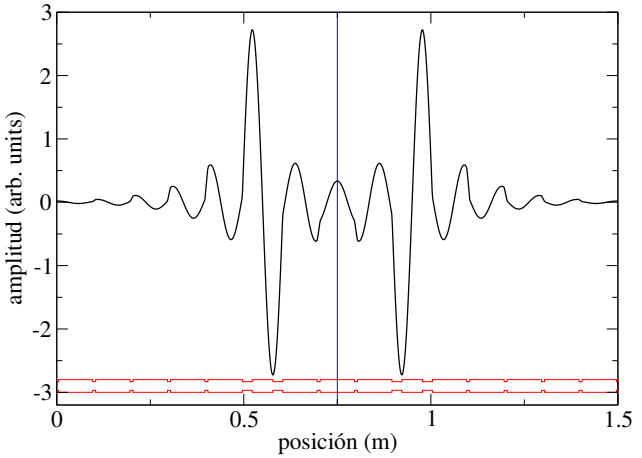

(b)

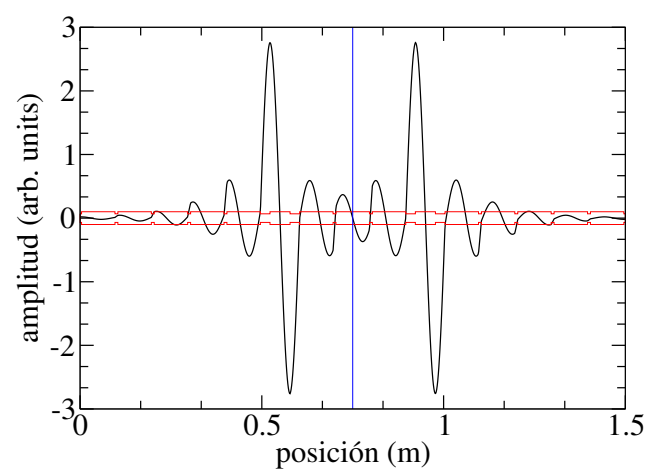

(c)

Figura 3.17: En (a) se muestra el espectro de frecuencias alrededor de la segunda brecha para el sistema $h=0.4$. En (b) y (c) se muestra una amplitud de onda del sistema de 5 celdas con 1 y 2 defectos correspondientes a las dos frecuencias de la banda emergente en la brecha.

\section{Defecto}

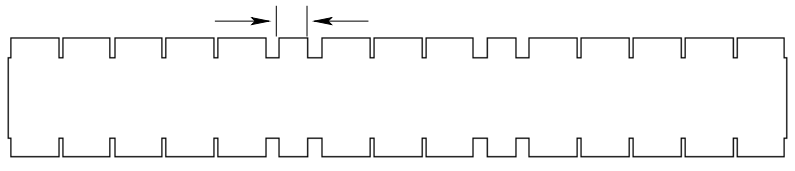

Figura 3.18: (a): Sistema localmente periódico con dos defectos, número de celdas simples $(\mathrm{nc})=15$, longitud total $(\mathrm{L})=1.5 \mathrm{~m}$, ancho de la muesca $($ eps $)=8 \mathrm{~mm}$, razón entre la muesca y la costilla $(\eta)=0.68$.

$\mathrm{Su}$ espectro de frecuencias de este sistema se muestra en la figura 3.19 


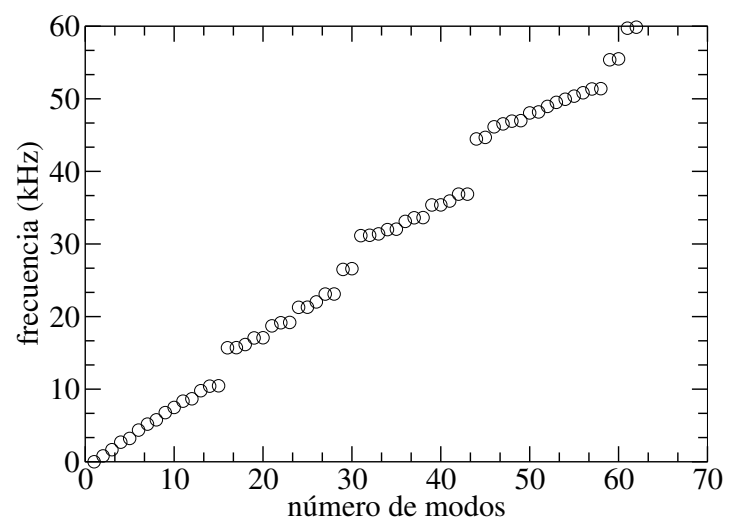

Figura 3.19: Espectro de frecuencias asociadas a modos torsionales. Note que hay dos niveles en el 2do y 4to gap asociados con los dos defectos.

En la estructura de bandas del correspondiente espectro de frecuencias del sistema 3.18 , podemos observar 2 niveles de frecuencia en la segunda brecha. Dichos niveles están asociados a los primeros estados excitados de los dos defectos. También existen 2 frecuencias en el cuarta brecha, pero no los elegimos debido a que estas frecuencias no pueden ser medidas por nuestro equipo experimental (amplificador de audio). Las amplitudes de onda de dichos niveles se muestran en la figura 3.20.

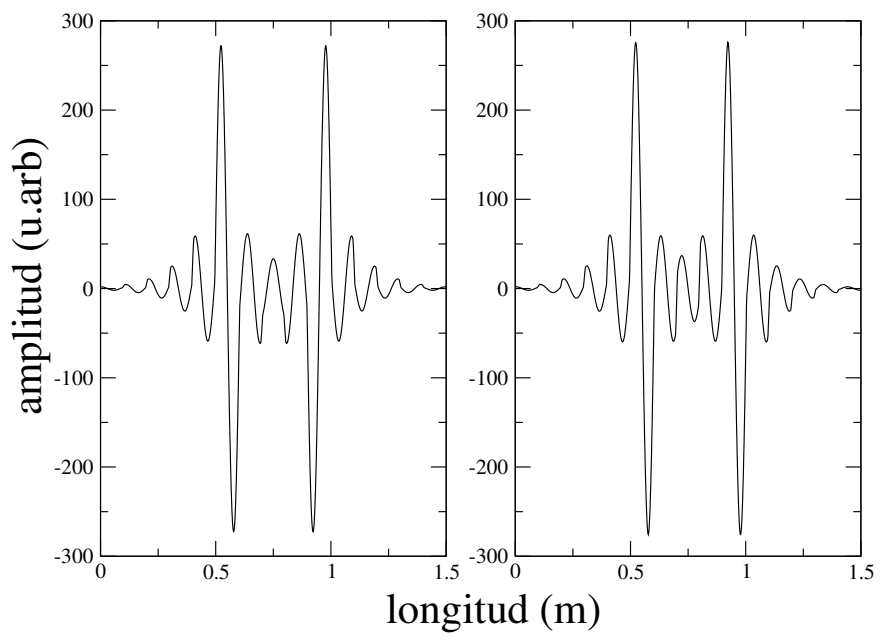

Figura 3.20: Amplitudes de onda asociadas a los modos 59 y 60, de la estructura de la figura 3.18 que aparecen en el segundo gap.

La amplitud de onda de la izquierda es simétrica y la de la derecha es antisimétrica (ver figura 3.20), se puede ver que ambas amplitudes de onda tienen amplitud máxima en el centro de cada una de las superceldas. A continuación se hará el mismo análisis para el mismo sistema sólo que ahora será un sistema con un defecto (ver figura 3.10). La 
estructura de bandas espectral del sistema con 2 defectos es comparada con la del sistema de un defecto abajo.

El sistema localmente periódico que cumple con lo que buscamos tiene una $h=0.4$ (ver 3.18). Dicho sistema tiene los dos niveles dentro de la brecha (ver figura 3.19). Además tiene amplitudes de onda localizadas (ver figura 3.20). Estas dos características sirven para el modelo (del cual se hablará más adelante) y también sirven para realizar el estudio experimental. El diseño de la celda unitaria comienza con la ranuración de una celda simple; es decir, se va a ranurar una de las costillas más grande por ambos lados (ver figura 3.9), de tal forma que al analizar los espectros de frecuencias estos cumplan con una característica muy específica; es decir, que dos niveles se despeguen de una de las bandas y se vayan al gap (ver figura 3.16b). Cabe mencionar que, además de determinar las dimensiones del grosor, también se determinó la longitud de la supercelda, el número de segmentos de la cual tenía que estar compuesta la supercelda y ancho de cada una de las muescas.

\subsection{Sistema localmente periódico con varias super- celdas acopladas}

Una vez comprobado que los defectos introducen la fenomenología de TB se diseñaron 6 sistemas elásticos con una y hasta 6 superceldas elásticas acopladas. Estos sistemas se diseñaron a partir del método MMT (matriz de transferencia). En la figura 3.21a) se muestra un corte longitudinal de dichos sistemas. Se calcularon numéricamente los espectros de los sistemas elásticos correspondientes a: una supercelda, dos superceldas acopladas y así sucesivamente hasta el sistema con seis superceldas acopladas. Las frecuencias de modo normal de cada uno de los sistemas de la figura 3.21a, se muestra en la gráfica de la figura 3.21b. Uno de los principales resultados de este trabajo se muestra claramente en el surgimiento de una nueva banda dentro de la segunda brecha del sistema periódico original de celdas simples, como resultado de la supercelda diseñada. Los niveles de frecuencia en esta banda emergente, se distribuyen alrededor del primer nivel que apareció en la brecha para el sistema de una sola supercelda, ver figura 3.21b. Esto es completamente diferente del caso del cristal elástico de celda simple considerado en la referencia [6]. Este comportamiento es similar al espectro de bandas de cristales atómicos. 


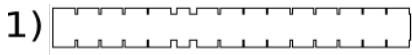

2)

3)

4) 1, 1,

5)

6)

)

(a)

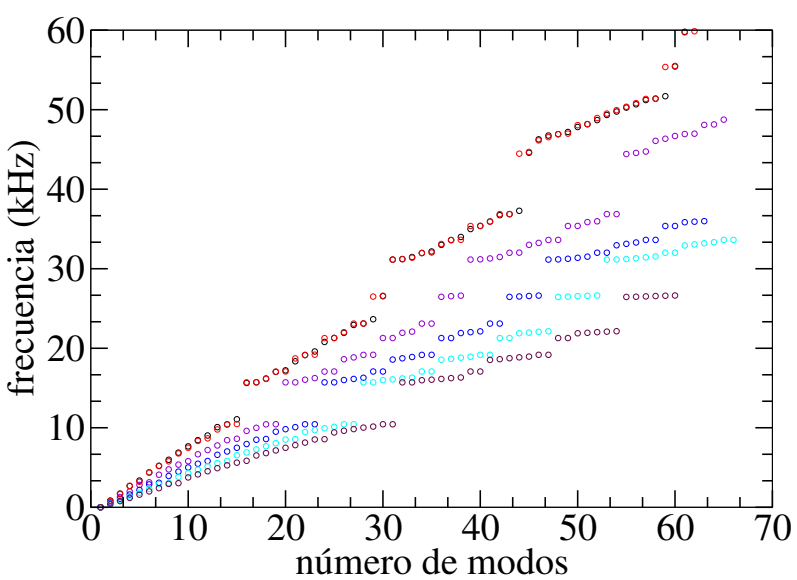

(b)

Figura 3.21: a): Corte longitudinal de los sistemas elásticos desde una hasta 6 superceldas elásticas acopladas; b): Note la banda emergente en la segunda brecha, alrededor de los $26 \mathrm{kHz}$.

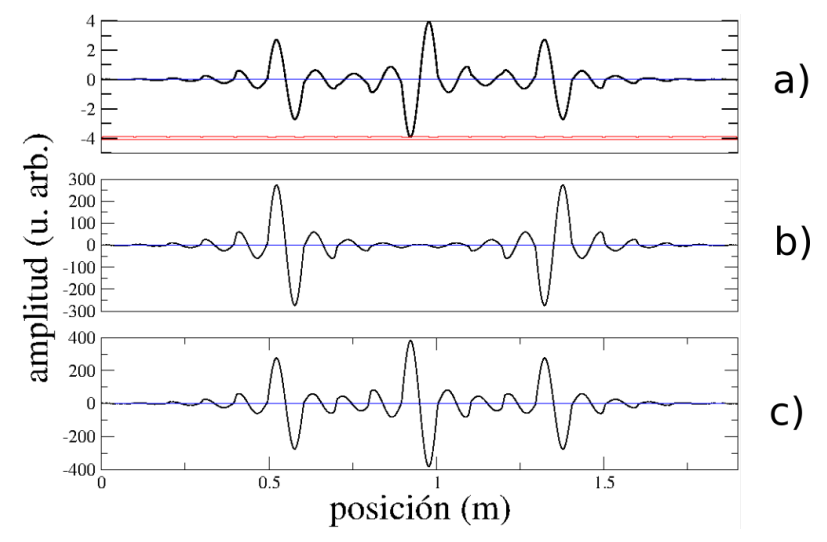

Figura 3.22: Amplitudes de onda, en u. arb. como función de la posición en m., asociadas al sistema de los 3 defectos acoplados, de la figura 3.21a, correspondiente a los modos 36, 37 y 38 de los niveles en el gap, de la figura $3.21 \mathrm{~b}$, con a) $f_{36}=26.472237 \mathrm{kHz}$, b) $f_{37}=$ $26.544451 \mathrm{kHz}$ y c) $f_{45}=26.613457 \mathrm{kHz}$

En la figura 3.22 se muestran las amplitudes de onda asociadas a los niveles de la banda emergente para una barra con tres superceldas acopladas. En la primera gráfica de la figura 3.22 se puede ver que la máxima amplitud se sitúa en el centro de las 3 superceldas (SC). Además se observa que es una amplitud de onda antisimétrica. En la segunda gráfica de la figura 3.22 se puede ver que solo hay máximos en la primer y tercer supercelda, mientras que en la segunda SC la amplitud es mínima. En la tercera gráfica de la figura 3.22 se puede ver que hay máximos que están localizados en el centro de las $3 \mathrm{SC}$ y que la amplitud de onda es también antisimétrica. 
Las amplitudes numéricas asociadas a un sistema con cuatro superceldas y a los niveles de la banda emergente, se muestra en la figura 3.23.

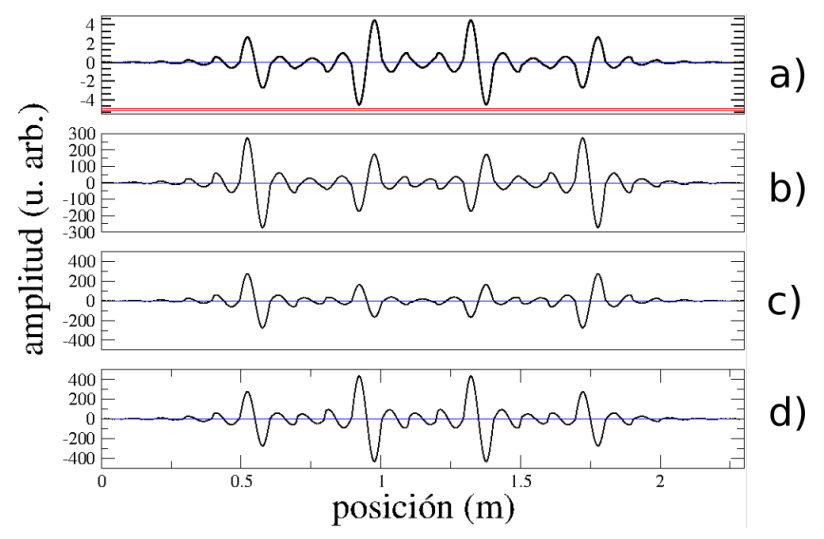

Figura 3.23: Amplitudes de onda asociadas al sistema de las 4 superceldas acopladas (ver $3.21 \mathrm{~b}$ ) correspondiente a $\operatorname{los} \operatorname{modos} 43,44,45$ y 46 de los niveles en el gap, con a) $f_{43}=$ $26461.323 \mathrm{~Hz}, \mathrm{~b}) f_{44}=26512.724 \mathrm{~Hz}$, c) $f_{45}=26574.426 \mathrm{~Hz}$ y d) $f_{46}=26622.941 \mathrm{~Hz}$

Los patrones estacionarios de la figura 3.23 tienen 4 máximos alrededor del centro de cada supercelda. En cada uno de estas figuras se muestra la posición $x$ de los máximos para cada una de las frecuencias. Asimismo en la figura se puede ver la contribución de la base numérica $\phi_{n}(x)$; es decir, la $\theta(x)$.

Las amplitudes de onda numéricas para un sistema de $5 \mathrm{SC}$ (ver 3.21b) se muestran en la figura 3.24.

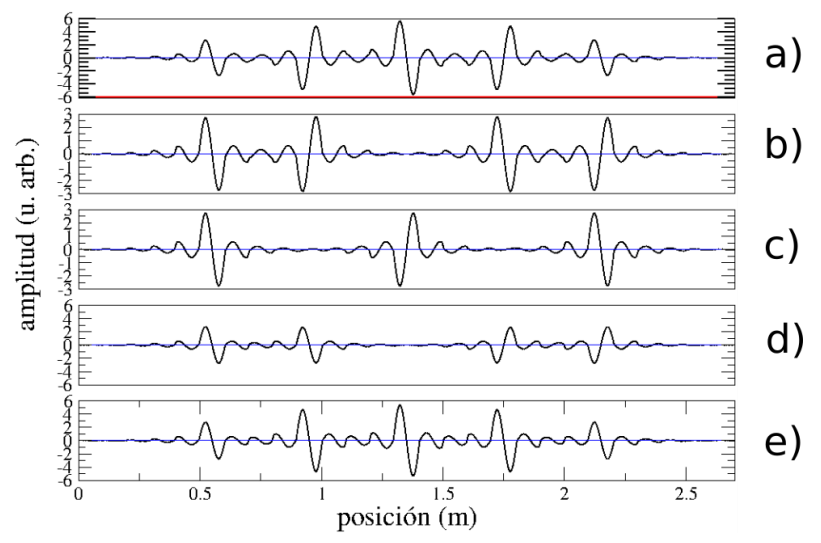

Figura 3.24: Amplitudes de onda asociadas al sistema de las 5 superceldas acopladas correspondientes a los modos 48, 49, 50, 51 y 52 de los niveles en el gap, con a) $f_{48}=$ $26455.266 \mathrm{~Hz}, \mathrm{~b}) f_{49}=26492.932 \mathrm{~Hz}$, c) $f_{50}=26543.401 \mathrm{~Hz}$, d) $f_{51}=26592.791 \mathrm{~Hz} \mathrm{y} \mathrm{e)}$ $f_{52}=26628.293 \mathrm{~Hz}$ 
Del mismo modo ocurre para el sistema con 5 defectos de la figura 3.24, donde el patrón estacionario completo de cada gráfica asociado a cada frecuencia, se forma gracias a la contribución de la base $\phi_{n}(x)$ (ver figura 3.13) asociada a un solo defecto.

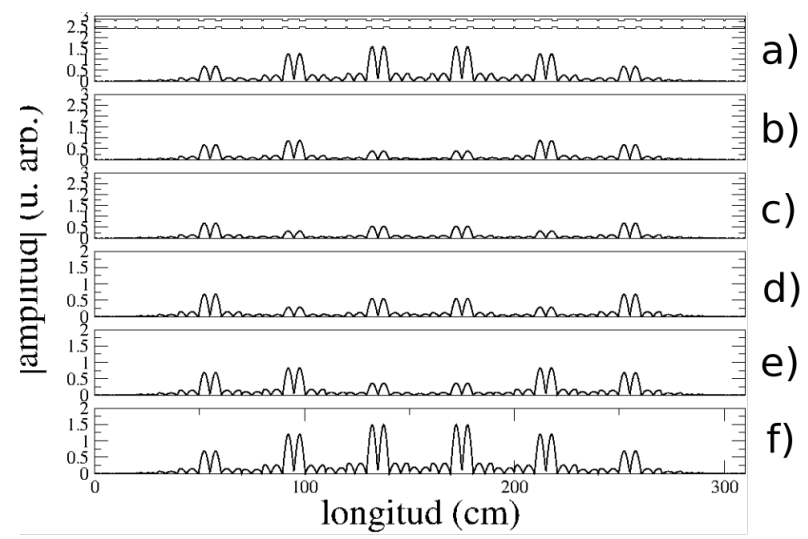

Figura 3.25: Amplitudes de onda asociadas al sistema de las 6 superceldas acopladas correspondientes a los modos 55, 56, 57, 58, 59 y 60 de los niveles en el gap, con a) $\left.f_{55}=26451.58 \mathrm{~Hz}, \mathrm{~b}\right) f_{56}=26480.109 \mathrm{~Hz}$, c) $\left.\left.f_{57}=26520.79 \mathrm{~Hz}, \mathrm{~d}\right) f_{58}=26565.227 \mathrm{~Hz}, \mathrm{e}\right)$ $f_{59}=26604.648 \mathrm{~Hz} \mathrm{y} \mathrm{f)} f_{60}=26631.598 \mathrm{~Hz}$

El objetivo principal de la sección que acabamos de ver fue el diseño de una supercelda unitaria. Con dicha supercelda podemos construir un sistema elástico 1D localmente periódico con propiedades de un cristal atómico. Además, logramos obtener el espectro de frecuencias y las amplitudes de onda de diferentes sistemas. El próximo capítulo trata de la comprobación de dichos sistemas diseñados, con un enfoque tanto numérico como experimental. La simulación numérica se hace con un modelo empírico de acoplamiento elástico, mientras que las mediciones se hacen usando un método llamado espectroscopia acústica resonante. 


\section{Capítulo 4}

\section{Diseño numérico del cristal elástico usando el modelo de acoplamiento elástico}

Una vez diseñado los 5 sistemas elásticos localmente periódicos con el MMT en el capítulo anterior, lo que sigue es comparar el espectro de frecuencias de dichos sistemas con el espectro obtenido para los mismos sistemas, pero usando el modelo de enlace fuerte elástico. El objetivo es probar que dicho modelo funciona sin importar el número de defectos. En este capítulo se explica el modelo de enlace fuerte elástico y se presentan los resultados arrojados por el mismo. Los sistemas analizados abarcan desde un sistema con 2 defectos acoplados hasta un sistema con 100 defectos acoplados.

\subsection{Modelo de enlace fuerte elástico empírico para describir el cristal elástico diseñado}

Para desarrollar un modelo empírico de acoplamiento elástico para ondas torsionales, en un material estructurado utilizamos simulaciones numéricas basadas en el método de la matriz de transferencia (TMM) en una estructura localmente periódica cuasi 1D, de la estructura propuesta. En la figura 4.1 se muestra la estructura elástica de una supercelda o celda unitaria compleja, previamente diseñada en el capítulo anterior, centrada en el sitio $i$ de una malla $1 \mathrm{D}$. 


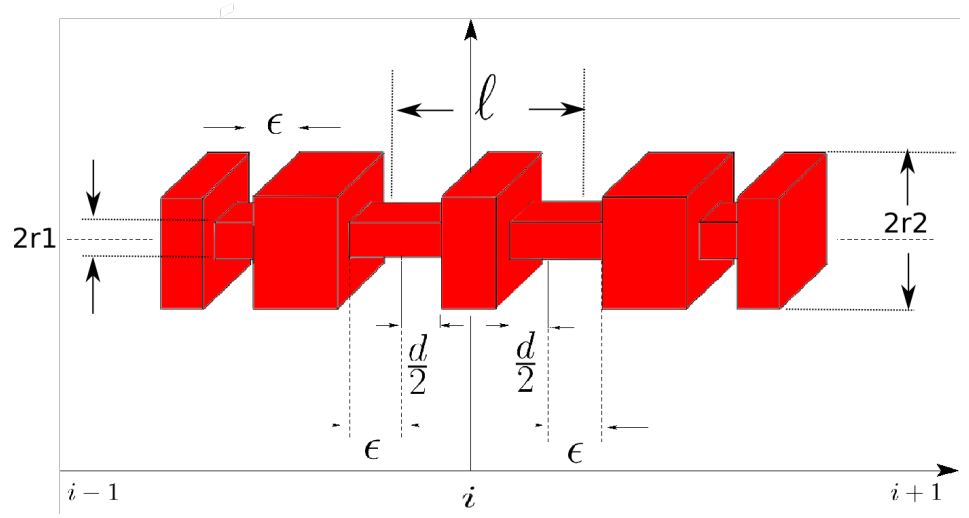

Figura 4.1: Celda unitaria elástica compleja centrada en la posción $i$ de la red periódica 1-D. Sus dimensiones estan determinados por los valores de los parámetros $d$ y $\epsilon$.

Esta figura muestra un arreglo de resonadores elásticos de sección transversal cuadrada. El número óptimo de resonadores (cinco) y sus dimensiones de los mismos, se determinaron numéricamente en el capítulo anterior. Este sistema de una impureza corresponde a una varilla de aluminio, cuyo grosor inicial es $1 / 2^{\prime \prime}$ (como las vende el fabricante). Este arreglo se logró variando el grosor de la varilla, como función del espacio, según el diseño. Así 5 de los bloques mantienen el grosor de la sección transversal original, mientras que los otros 4 fueron devastados. El bloque central de esta supercelda tiene una longitud diferente a la de un resonador típico en la estructura por lo que le llamamos defecto o impureza. Cabe mencionar que además de determinar las dimensiones del grosor, también se determinaron todos los parámetros según el diseño numérico como el número de segmentos y el ancho de cada una de las muescas de dicho sistema, de tal forma que se pueda medir en el audible y ser fabricado. Las simulaciones numéricas también consideraron las propiedades del material 6061-T6. La estructura final (cristal localmente periódico) es invariente ante traslaciones sobre el eje x para el tamaño de la celda. Es importante notar que el sistema con una impureza elegida para su fabricación no es único, pero se eligió así para obtener las características deseadas. Un corte longitudinal de un sistema periódico con un defecto se muestra en la figura 4.2. 


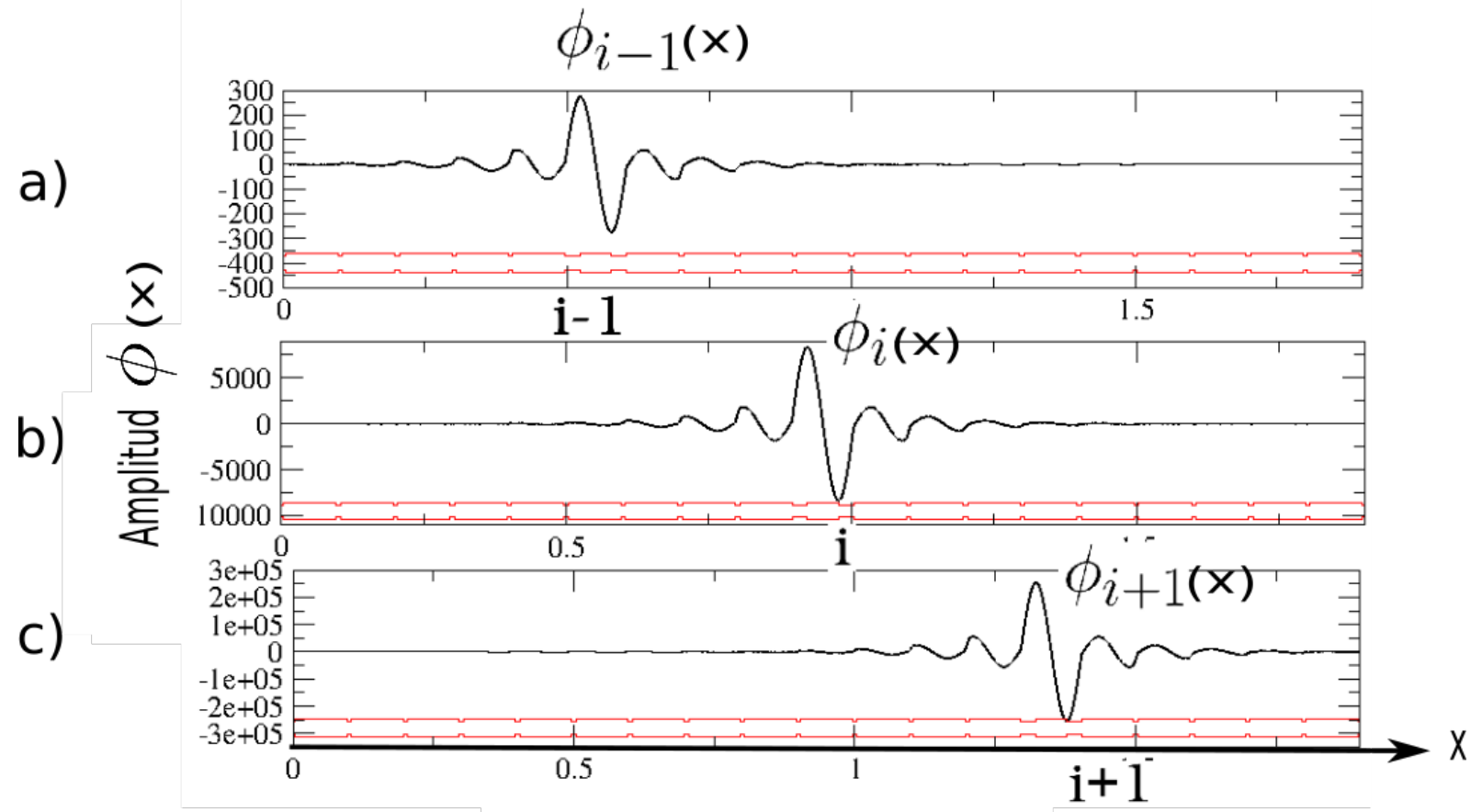

Figura 4.2: En rojo se muestra un corte longitudinal de una varilla con estructura periódica de celda unitaria simple con un defecto incluido en el sitio a) $i-1$ b) $i$ y c) $i+1$. En negro se muestra la amplitud de onda torsional asociada al sistema de una supercelda elástica aislada, ubicada en el sitio a) $i-1$ b) $i$ y c) $i+1$ sobre una estrutura periódica de $\mathrm{N}$ celdas unitarias. La frecuencia de sitio es $f_{0}=25886.9 \mathrm{~Hz}$ y es independiente del sitio de ubicación de la supercelda. Estas amplitudes de onda localizadas alrededor de los sitios a) $i-1$, b) $i$ y c) $i+1$ son elementos de la base $\left\{\phi_{i}(x), i=1,2, \ldots\right\}$. Donde a) $\left.\phi(x)_{i-1}, \mathrm{~b}\right)$ $\phi(x)_{i}$ y c) $\phi(x)_{i+1}$ son la amplitudes de onda tosionales localizadas en el sitio a) $i-1$, b) $i$ e c) $i+1$, respectivamente. 


\subsubsection{Acoplamiento de 2 superceldas elásticas}

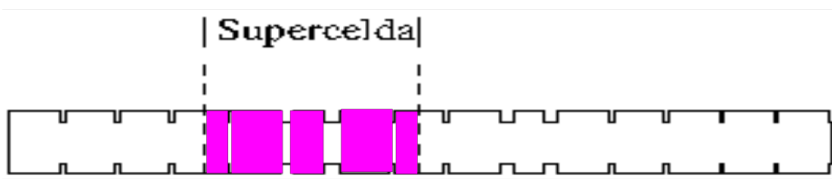

a)

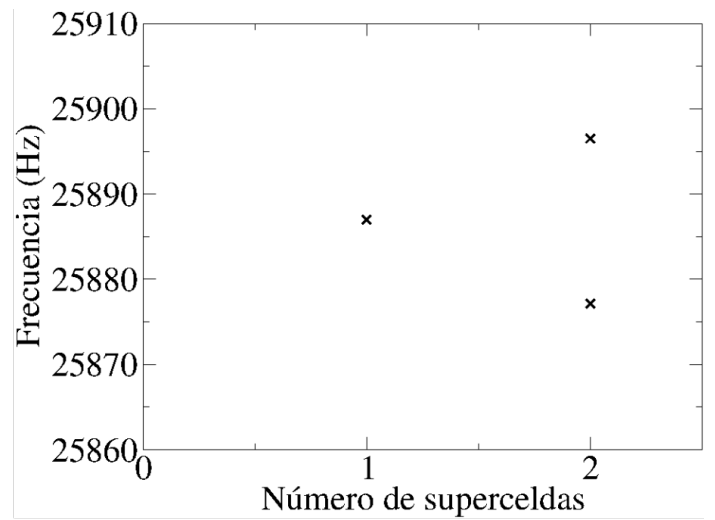

b)

Figura 4.3: (a) Corte longitudinal de una estructura elástica periódica de 15 celdas unitarias con 2 defectos. (b): Espectro de frecuencias propias asociados a ondas torsionales del sistema elástico en (a). Estos niveles aparecen en el segundo gap del sistema periódico original a consecuencia de los defectos como función del número de superceldas.

Las frecuencias de resonancia de los dos sistemas elásticos de una y dos superceldas asociadas a una nueva banda emergente en la segunda brecha del sistema periódico original se ven en la figura 4.3 como función del número de superceldas. Los niveles para el caso de 2 superceldas muestran simetría alrededor del nivel asociado a una supercelda aislada. Esto es una característica de los espectros de cristales atómicos. La amplitud de onda asociada al nivel para el caso de una sola supercelda se ve en la figura 4.2. Note que la amplitud de onda decae evanescentemente hacia ambos lados; por lo tanto, solo traslapa (acopla) con sus primeros vecinos; en otras palabras, la amplitud de onda está localizada en el sitio $i$. Esto permite proponer un modelo empírico de acoplamiento elástico, similar al cuántico, para ondas torsionales a primeros vecinos tal que, el sistema de ecuaciones está determinado por:

$$
-C A_{i-1}+f_{i} A_{i}-C A_{i+1}=f A_{i}
$$

Recordando que, $f_{i}$ es la frecuencia de resonancia de la supercelda aislada ubicada en el sitio $i, C$ son los elementos de la matriz que acopla superceldas vecinas; también pueden interpretarse como los acoplamientos entre dos superceldas que dependen de la frecuencia de resonancia de la supercelda aislada y de la distancia entre las superceldas considerando que el acoplamiento es constante; $f$, es la frecuencia propia de vibración del sistema completo de superceldas acopladas y $A_{i}$ es el coeficiente i-ésimo de la expansión $\theta(x)$, en una base $\phi_{i}(x), i=1,2, \ldots$ de estados de cada uno de los defectos aislados (ver figura 4.2 y 4.4). 
Este modelo nos permite expandir, en el caso más sencillo, las amplitudes de onda del sistema de superceldas interactuantes, en términos de las amplitudes de onda $\phi_{i}(x)$ de un sistema de una supercelda aislada localizada en los sitios de una estructura localmente periódica, más el acoplamiento a primeros vecinos.

a)


Figura 4.4: Arriba: Sólido cristalino en una dimensión. Los átomos son posicionados en los sitios de la red $x=n a, n=0,1,2, \ldots$ (circulos oscuros). El traslape de las funciones de onda asociadas a electrones aislados en posiciones vecinas define el término de acoplamiento $C$ en el modelo de tight binding en un cristal atómico unidimensional

En lo que sigue se considere que el conjunto de frecuencias de su sitio $f_{i}: i=1,2 \ldots$ son idénticas; esto es: $f_{0}=f_{1}=f_{2}=f_{i}$ ó $\left\{f_{i}\right\}$ y $\left\{\phi_{i}\right\}$, con $i=1,2 . . N$. La base en la que se están expandiendo las funciones de onda del sistema acoplado son las de cada supercelda aislada.

$$
\theta(x)=\sum_{i}^{N} A_{i} \phi_{i}(x)
$$

Donde $\theta(\mathrm{x})$ es la amplitud de onda asociada al ángulo de torsión. Note que el modelo 
anterior es muy similar al modelo de enlace fuerte unidimensional usado en mecánica cuántica (ver referencia [34] ó capítulo 2).

Los parámetros relevantes del modelo de acoplamiento elástico $\left(f_{0} \mathrm{y} C\right)$ pueden fijarse a partir de las simulaciones numéricas, para una y dos super celdas, de la siguiente manera. A continuación se muestra el sistema con una, dos y tres superceldas acopladas.

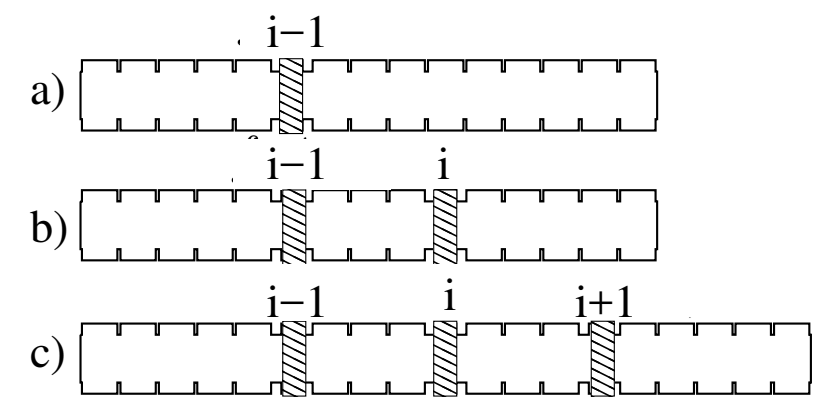

Figura 4.5: Tres sistemas estructurados: a) Periódico con un defecto en el sitio $i-1, \mathrm{~b}$ ) periódico con dos defectos en el sitio $i-1$ y $i$ c) periódico con tres defectos $i-1, i$ y $i+1$.

La ecuación (4.1) para un sólo defecto en el sitio $i$ queda así:

$$
A_{i} f_{0}=A_{i} f
$$

En la parte superior de la figura 4.5 a) se muestra el sistema con una supercelda. El espectro de frecuencias para dicho sistema se muestra en la figura 4.6.

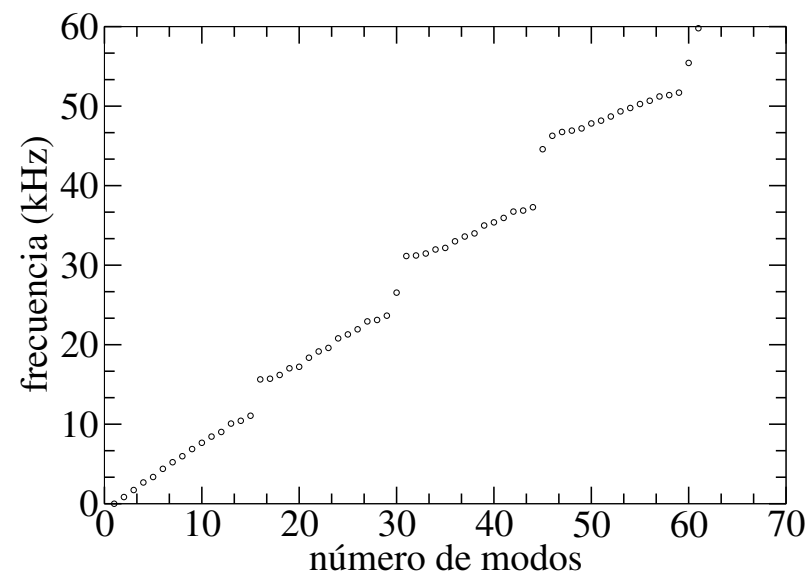

Figura 4.6: Espectro de la estructura periódica con un defecto o supercelda aislada en el sitio $i$.

$$
f_{0}=25886.99 H z
$$


Esta $f_{0}$ es determinada numéricamente y corresponde a la frecuencia $f_{0}$. Considere ahora, el sistema de dos superceldas acopladas de la figura 4.5, el sistema de ecuaciones (4.1) queda:

$$
\begin{aligned}
f_{0} A_{1}-C A_{2} & =f A_{1}, \\
-C A_{1}+f_{0} A_{2} & =f A_{2} .
\end{aligned}
$$

Este sistema de ecuaciones es un problema de valores y vectores propios.

$$
\begin{gathered}
\left(\begin{array}{cc}
f_{0} & -C \\
-C & f_{0}
\end{array}\right)\left(\begin{array}{l}
A_{1} \\
A_{2}
\end{array}\right)=f\left(\begin{array}{l}
A_{1} \\
A_{2}
\end{array}\right) \\
\operatorname{det}\left(\begin{array}{cc}
f_{0}-f & -C \\
-C & f_{0}-f
\end{array}\right)=0 \\
\left(f_{0}-f\right)^{2}=C^{2},
\end{gathered}
$$

de manera que las frecuencias propias son:

$$
\begin{aligned}
& f_{+}=f_{0}+C \\
& f_{-}=f_{0}-C
\end{aligned}
$$

De las ecuaciones (4.8) y (4.9) se obtiene que $C$, esto es:

$$
C=\Delta f / 2, \quad \text { con } \quad \Delta f=f_{+}-f_{-},
$$

siendo $f_{+}$y $f_{-}$las frecuencias correspondientes a los dos niveles en el segundo gap.

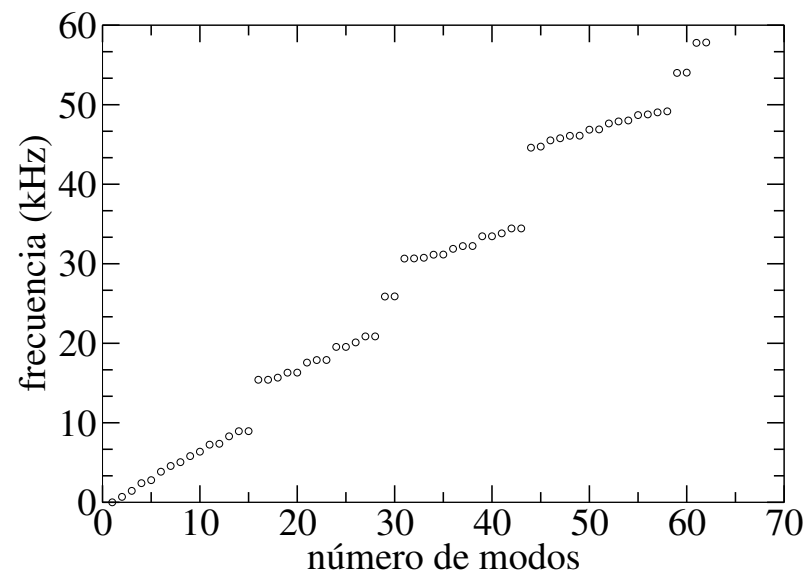

Figura 4.7: Espectro de frecuencias torsionales del sistema periódico con 2 defectos. Ver figura $4.5 \mathrm{~b})$.

Estos valores de $f_{0}$ y $C$ comparada con el sistema periódico original quedan definidos de la simulación numérica por el método de la matriz de transferencia (MMT) (ver figura 
4.3). Así; $f_{0}=f=25886.99 \mathrm{~Hz}$ y $C=9.687 \mathrm{~Hz}$. Con estos valores el modelo de enlace fuerte elástico queda completamente determinado de manera que obtenemos:

$$
\begin{aligned}
& f_{+}=25886.99 H z+9.68 H z=25896.68 H z \\
& f_{-}=25886.99 H z-9.68 H z=25877.30 H z
\end{aligned}
$$

Mientras que, la simulación numérica indica (según las ecuaciones ((4.8) y (4.9)) que $f_{-}=25896.51 \mathrm{~Hz}$ y $f_{+}=25877.14 \mathrm{~Hz}$.

Los vectores propios, por su parte se obtienen de las ecuaciones (4.4) y con (4.8)

$$
\begin{gathered}
f_{0} A_{1}-C A_{2}=\left(f_{0}+C\right) A_{1}, \\
A_{1}=-A_{2} .
\end{gathered}
$$

De las ecuaciones (4.4) y con (4.9)

$$
\begin{aligned}
f_{0} A_{1}-C A_{2} & =\left(f_{0}-C\right) A_{1}, \\
A_{2} & =A_{1} .
\end{aligned}
$$

Los eigenvectores normalizados quedan:

$$
\vec{v}_{+}=\frac{1}{\sqrt{2}}\left(\begin{array}{l}
1 \\
1
\end{array}\right) ; \quad \vec{v}_{-}=\frac{1}{\sqrt{2}}\left(\begin{array}{c}
1 \\
-1
\end{array}\right),
$$

para $f_{+}$y $f_{-}$, respectivamente.

La generalización de estos cálculos para el modelo del cristal elástico es como sigue:

$$
\vec{v}_{+}=\frac{1}{\sqrt{2}}\left\{\phi_{1}(x)+\phi_{2}(x)\right\} ; \quad \vec{v}_{-}=\frac{1}{\sqrt{2}}\left\{\phi_{1}(x)-\phi_{2}(x)\right\},
$$

El sistema de 3 superceldas acopladas es la primer prueba del modelo (ver figura 4.5 c)). La ecuación 4.1 queda como:

$$
\begin{array}{r}
f_{0} A_{1}-C A_{2}=f A_{1} \\
-C A_{1}+f_{0} A_{2}-C A_{3}=f A_{2} \\
-C A_{2}+f_{0} A_{3}=f A_{3} \\
\operatorname{det}\left(\begin{array}{lrr}
f_{0}-f & -C & 0 \\
-C & f_{0}-f & -C \\
0 & -C & f_{0}-f
\end{array}\right)=0 \\
\left(f_{0}-f\right)^{3}-2 C^{2}\left(f_{0}-f\right)=0
\end{array}
$$


Las frecuencias propias son:

$$
\begin{aligned}
& f_{1}=f_{0}-\sqrt{2} C \\
& f_{2}=f_{0}, \\
& f_{3}=f_{0}+\sqrt{2} C .
\end{aligned}
$$

Sustituyendo $f_{0}$ y $C$ en las ecuaciones (4.22)

$$
\begin{aligned}
& f_{1}=25896.51 H z-\sqrt{2}(9.68) H z=25873.29 H z \\
& f_{2}=25886.99 H z \\
& f_{3}=25896.51 H z+\sqrt{2}(9.68) H z=25900.69 H z
\end{aligned}
$$

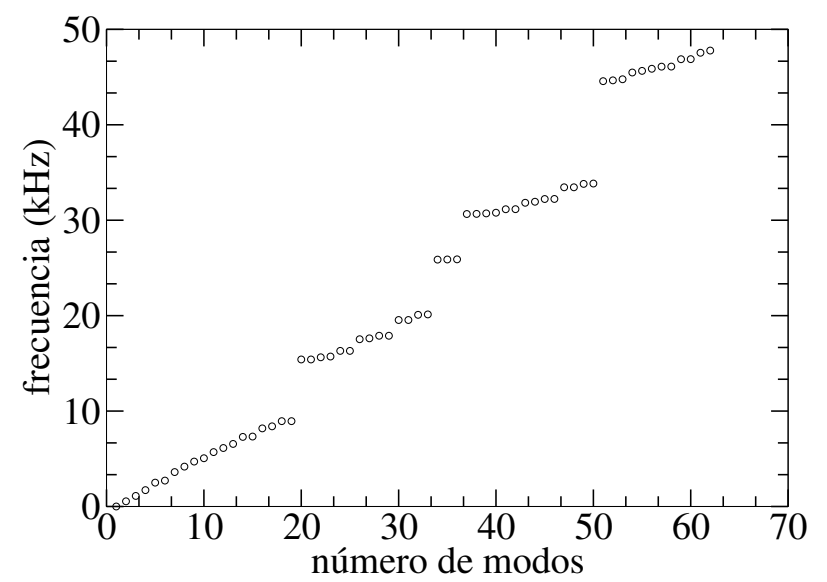

Figura 4.8: Espectro de frecuencias torsionales numérico para un sistema de 3 defectos acoplados.

Por último comparando los resultados 4.23 con la simulación numérica (MMT) (ver 4.8): $f_{1}^{(T M M)}=25873.05 \mathrm{~Hz}, f_{2}^{(T M M)}=25886.82 \mathrm{~Hz} \mathrm{y} f_{3}^{(T M M)}=25900.45 \mathrm{~Hz}$ se puede ver que los resultados numéricos obtenidos con la matriz de transferencia (figura 4.8) concuerdan de manera excelente (con un error del menos del 1\%)con los resultados teóricos (4.23).

Los vectores propios correspondientes son:

$$
\begin{array}{r}
A_{2}=\sqrt{2} A_{1}, \\
A_{3}=\frac{A_{2}}{\sqrt{2}} .
\end{array}
$$

De las ecuaciones (4.19) y con (4.22)

$$
\begin{gathered}
A_{2}=0, \\
A_{3}=A_{1} .
\end{gathered}
$$


De las ecuaciones (4.19) y con (4.22)

$$
\begin{aligned}
& A_{2}=-\sqrt{2} A_{1}, \\
& A_{3}=-\frac{1}{\sqrt{2}} A_{2} .
\end{aligned}
$$

Normalizando

$$
\overrightarrow{v_{1}}=\frac{1}{2}\left(\begin{array}{c}
1 \\
-\sqrt{2} \\
1
\end{array}\right), \quad \overrightarrow{v_{2}}=\frac{1}{\sqrt{2}}\left(\begin{array}{c}
1 \\
0 \\
-1
\end{array}\right), \quad \overrightarrow{v_{3}}=\frac{1}{2}\left(\begin{array}{c}
1 \\
\sqrt{2} \\
1
\end{array}\right) .
$$

Siguiendo un procedimiento similar a los anteriores se puede mostrar las frecuencias para un sistema de 4 defectos acoplados, ver tabla 4.1.

\begin{tabular}{|l|l||l||}
\hline frecuencias numéricas $(\mathrm{Hz})$ & frecuencias teóricas $(\mathrm{Hz})$ & error $(\%)$ \\
\hline \hline 25871.04 & 25881.96 & 0.04 \\
\hline 25880.79 & 25885.07 & 0.01 \\
\hline 25892.76 & 25888.92 & 0.01 \\
\hline 25902.39 & 25892.03 & 0.03 \\
\hline
\end{tabular}

Tabla 4.1: Comparación de frecuencias teóricas y numéricas para un sistema de 4 superceldas acopladas.

Como se puede ver el modelo elástico propuesto es muy exacto y para pocas superceldas es posible comparar los resultados numéricos con cálculos analíticos. Si el modelo puede reproducir, con similar precisión, sistemas elásticos de muchos defectos acoplados, permitirá reducir el tiempo de cálculo numérico, respecto al método de MT, del espectro de frecuencias para el cristal elástico. Esto es un logro importante, que es parte de la caracterización de sistemas elásticos. El modelo funciona para predecir cálculos espectrales de cristales elásticos. Este resultado es satisfactorio porque se ha demostrado que el modelo es una alternativa eficiente para calcular los modos normales de un cristal elástico y su correspondiente espectro en tiempos reducidos.

\subsection{Generalización del modelo de enlace fuerte elásti- co para un cristal infinito}

En esta sección se muestra que el modelo TB-elástico generalizado del modelo teórico de enlace fuerte es consistente con las simulaciones numéricas basadas en TMM. El modelo desarrollado para describir las ondas torsionales en un cristal elástico, construído a partir de un número infinito de superceldas acopladas esquematizada en la figura $4.9 \mathrm{y}$ en la ecuación 4.1. 


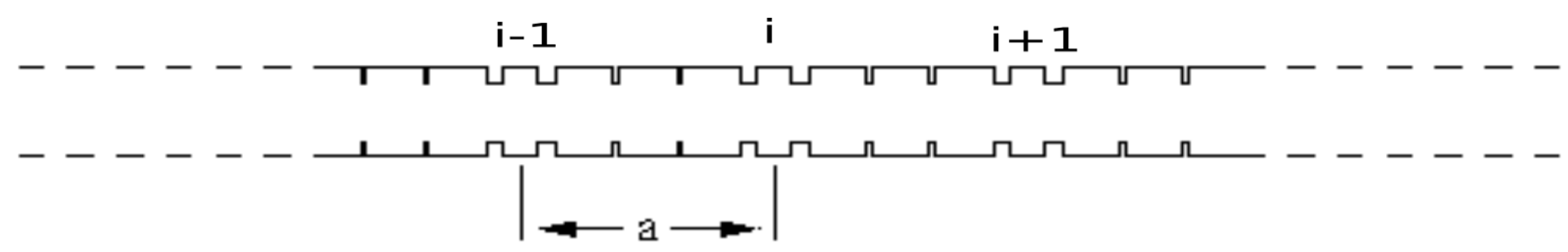

Figura 4.9: Cristal elástico ideal: Sistema infinito de superceldas elásticas acopladas.

Si consideramos el sistema periódico infinito, las amplitudes de onda, $A_{i}$, en los sitios $i-1, i$ y $i+1$ (ver figura 4.9) pueden escribirse:

$$
\begin{gathered}
A_{i}=e^{-i k a}, \\
A_{i-1}=A_{i} e^{i k a} \\
A_{i+1}=A_{i} e^{-i k a},
\end{gathered}
$$

donde " $a$ ", es la distancia entre dos sitios consecutivos (el periódo) y, " $k$ ", es el número de onda. Sustituyendo estos valores en la ecuación (4.1), se tiene que

$$
-C A_{i} e^{i k a}+f_{0} A_{i}-C A_{i} e^{-i k a}=f A_{i},
$$

y se puede mostrar que la relación de dispersión:

$$
f=f_{0}-2 C \cos (k a) .
$$

Recordando que $f$ son la frecuencias de resonancia del cristal; $f_{0}$ es la frecuencia de vibración de una supercelda aislada y $C$ es el acoplamiento. La gráfica de la relación de dispersión obtenida analíticamente a partir del modelo teórico propuesto, se muestra en la figura 4.10). Se confirma un buen acuerdo con los resultados del modelo teórico, descritos por la ecuación (ecuación 4.1). 


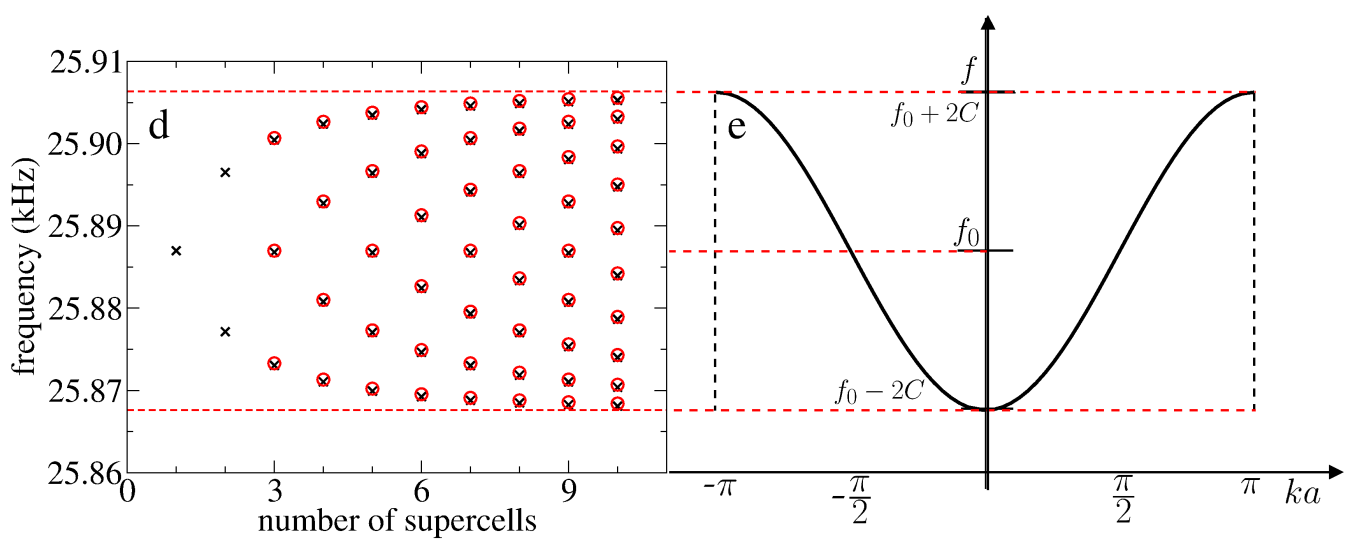

Figura 4.10: La nueva banda elástica emergente calculada en el segundo gap es acotada por las líneas punteadas horizontales. Estas corresponden a los valores máximos y mínimos de la frecuencia, según la relación de dispersión (ecuación (4.32)) para 10 superceldas elásticas acopladas mostrada a la derecha.

Con el fin de mostrar la capacidad y validez del modelo elástico T-B se realizó la simulación numérica para 50 superceldas acopladas y los resultados de la banda torsional emergente se muestran en la figura 4.11.

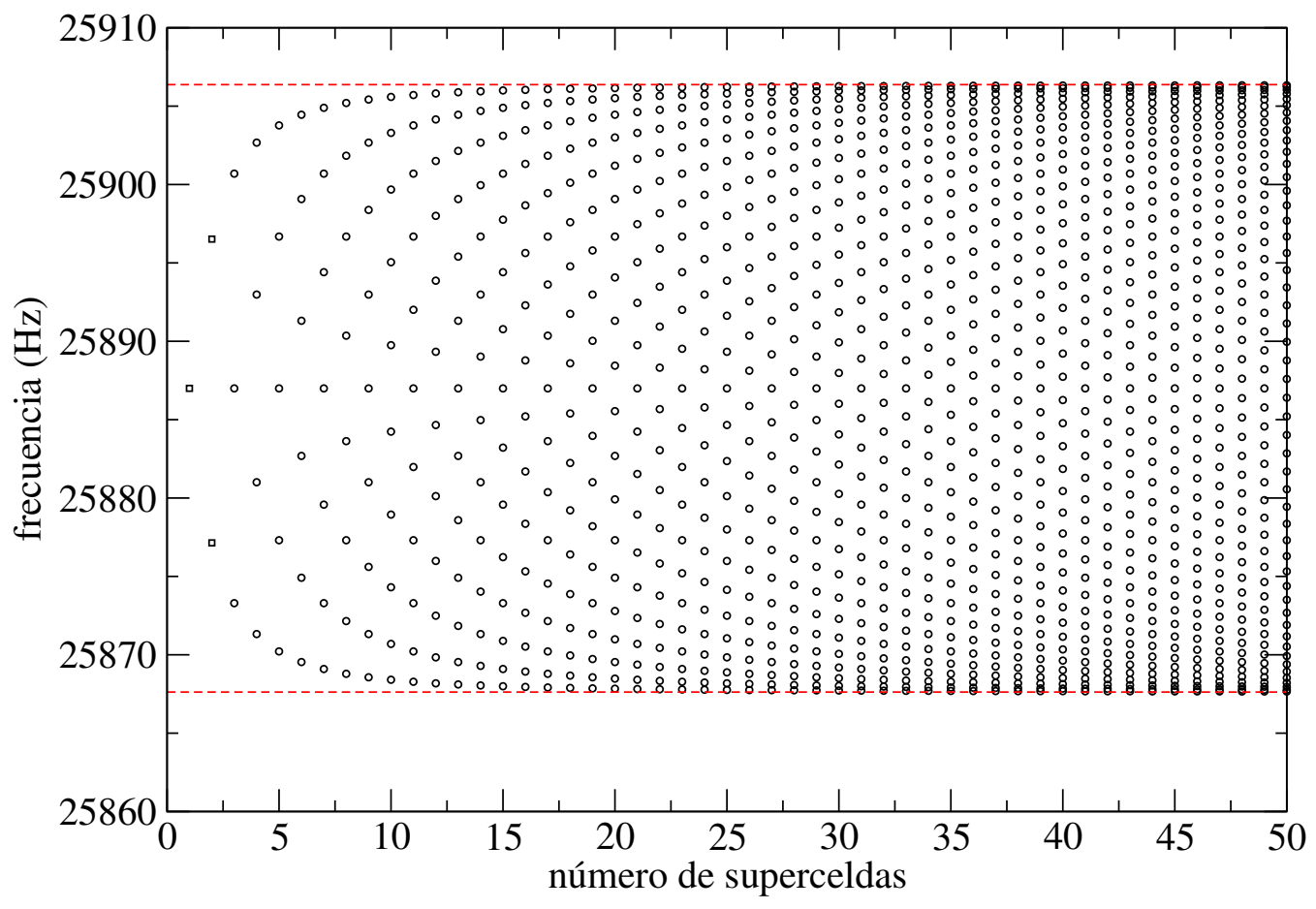

Figura 4.11: Banda elástica emergente del cristal elástico hasta para 50 superceldas elásticas acopladas. 
La gráfica mostrada en la figura 4.11 muestra la banda elástica emergente, para ondas torsionales del cristal elástico calculada a partir del modelo teórico hasta para 100 superceldas acopladas. Se puede ver que la banda está acotada por las frecuencias máxima y mínima, predichas por la correspondiente relación de dispersión. Esto comprueba que el modelo teórico de enlace fuerte propuesto para el cristal elástico es consistente. Esto es un logro importante ya que el cálculo de los espectros de frecuencia de cristales elásticos, usando este modelo, reducirá enormemente el tiempo de cálculo numérico en comparación con el método de la matriz de transferencia.

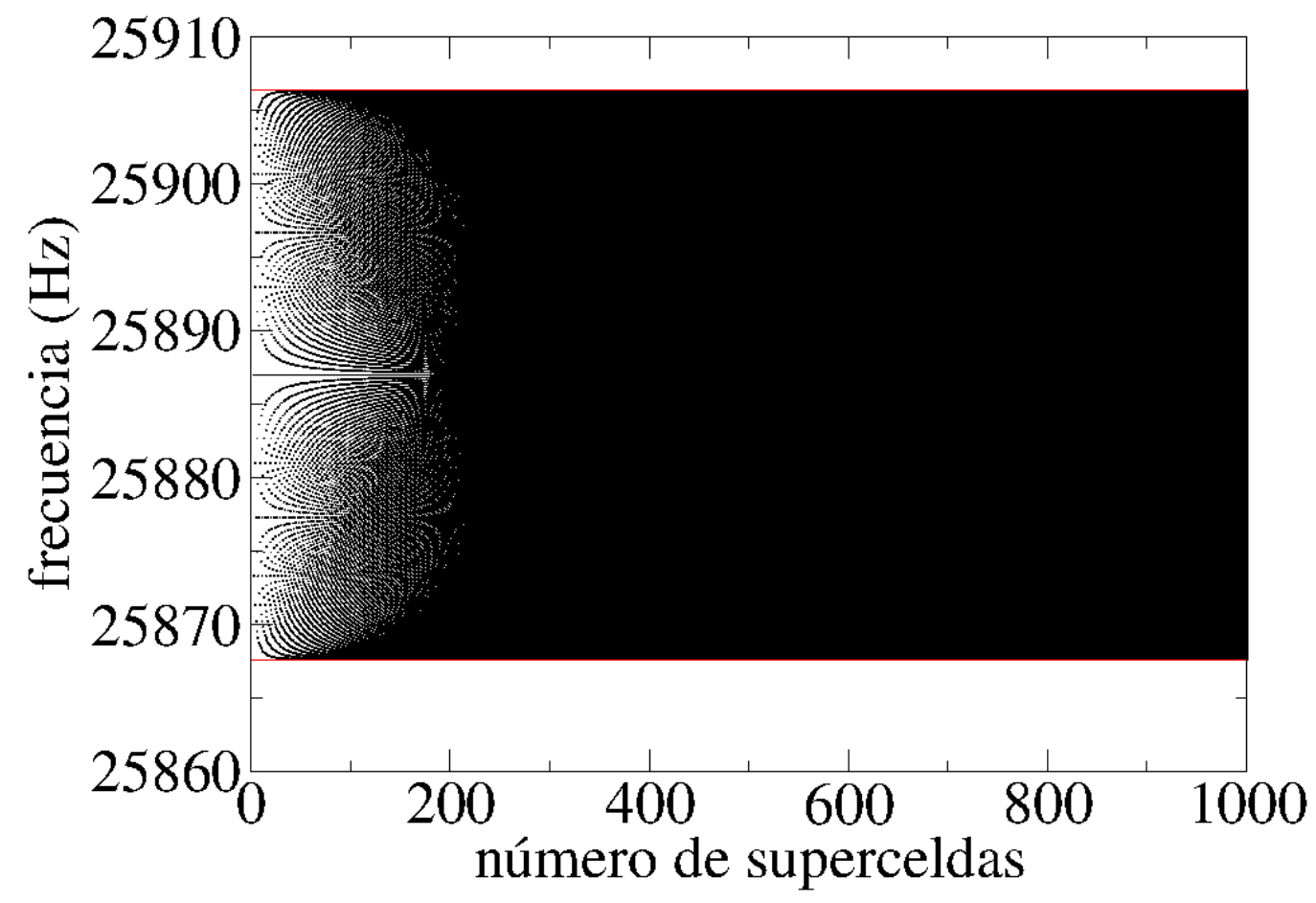

Figura 4.12: Banda elástica emergente del cristal elástico hasta para 1000 superceldas acopladas.

Otra forma de llegar a la ecuación 4.32 se muestra a continuación

$$
\begin{gathered}
C A_{n-1}+f_{0} A_{n}+C A_{n+1}=f A_{n} \\
A_{n}=e^{i k^{n} n} K^{n}=n \pi, n=0, \pm 1, \pm 2, \ldots \\
A_{n-1}=(-1)^{n+1} e^{i k^{(n)} n} e^{-i k^{(n)}} \\
A_{n+1}=(-1)^{n+1} e^{i k^{(n)} n} e^{i k^{(n)}}
\end{gathered}
$$




$$
\begin{gathered}
f=(-1)^{(n+1)} C e^{-i k^{n}}+f_{0}+(-1)^{(n+1)} C e^{i k^{(n)}} \\
f=(-1)^{(n+1)} C e^{-i n \pi}+f_{0}+(-1)^{(n+1)} C e^{i n \pi} \\
f=2(-1)^{n+1} C \cos (n \pi)+f_{0}
\end{gathered}
$$




\section{Capítulo 5}

\section{Resultados experimentales}

\subsection{Fabricación de los sistemas elásticos estructura- dos}

En esta sección se muestra el proceso de fabricación de los sistemas elásticos estructurados (cuasi-unidimensionales) de tamaño finito que se diseñaron para su caracterización, a partir de su espectro de frecuencia de las ondas mecánicas torsionales.

Para la fabricación de los sistemas cuasi-unidimensionales que se diseñaron en el capítulo anterior, se uso una máquina fresadora vertical. Esta puede ser programada por computadora para facilitar el trabajo de precisión requerido para maquinar las estructuras periódicas sobre varillas de aluminio 6061-T6. Dicha máquina fue pieza clave para la fabricación de los sistemas y se encuentra en el Instituto de Ciencias Físicas de la UNAM, en Cuernavaca Morelos.

En la figura 5.1 se muestran imágenes de la fabricación de los sistemas estructurados, sobre 5 varillas de sección transversal cuadrada, usando los diseños de la figura 3.21a, con una fresadora CNC. La fresadora garantiza una tolerancia de hasta $0.1 \mathrm{~mm}$ y asegura cifras significativas en el rango de las micras. Con ayuda de los controles Centroid M400 con que cuenta la fresadora, se programaron las dimensiones de los sistemas, dichos controles permiten manipular los 3 ejes espaciales.Los 5 sistemas periódicos fabricados se realizaron en un tiempo efectivo total de 5 semanas. 


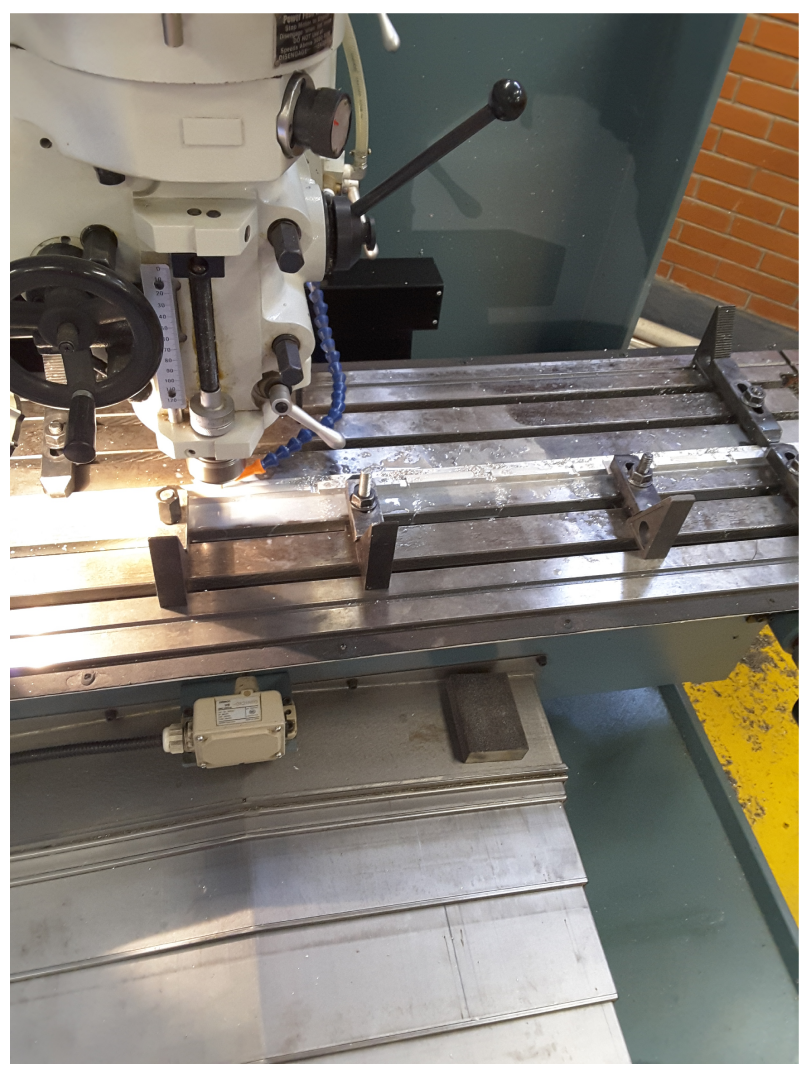

(a)

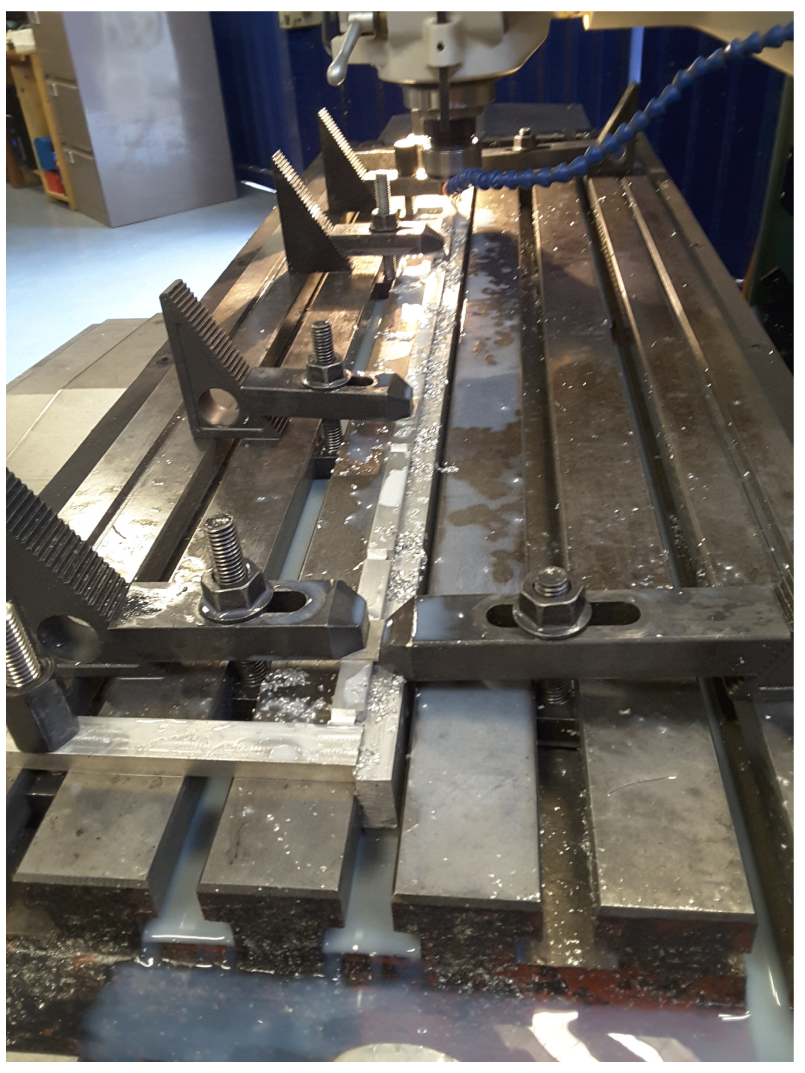

(b)

Figura 5.1: Maquinación, a): Fresadora CNC; b) Vista en perspectiva. 


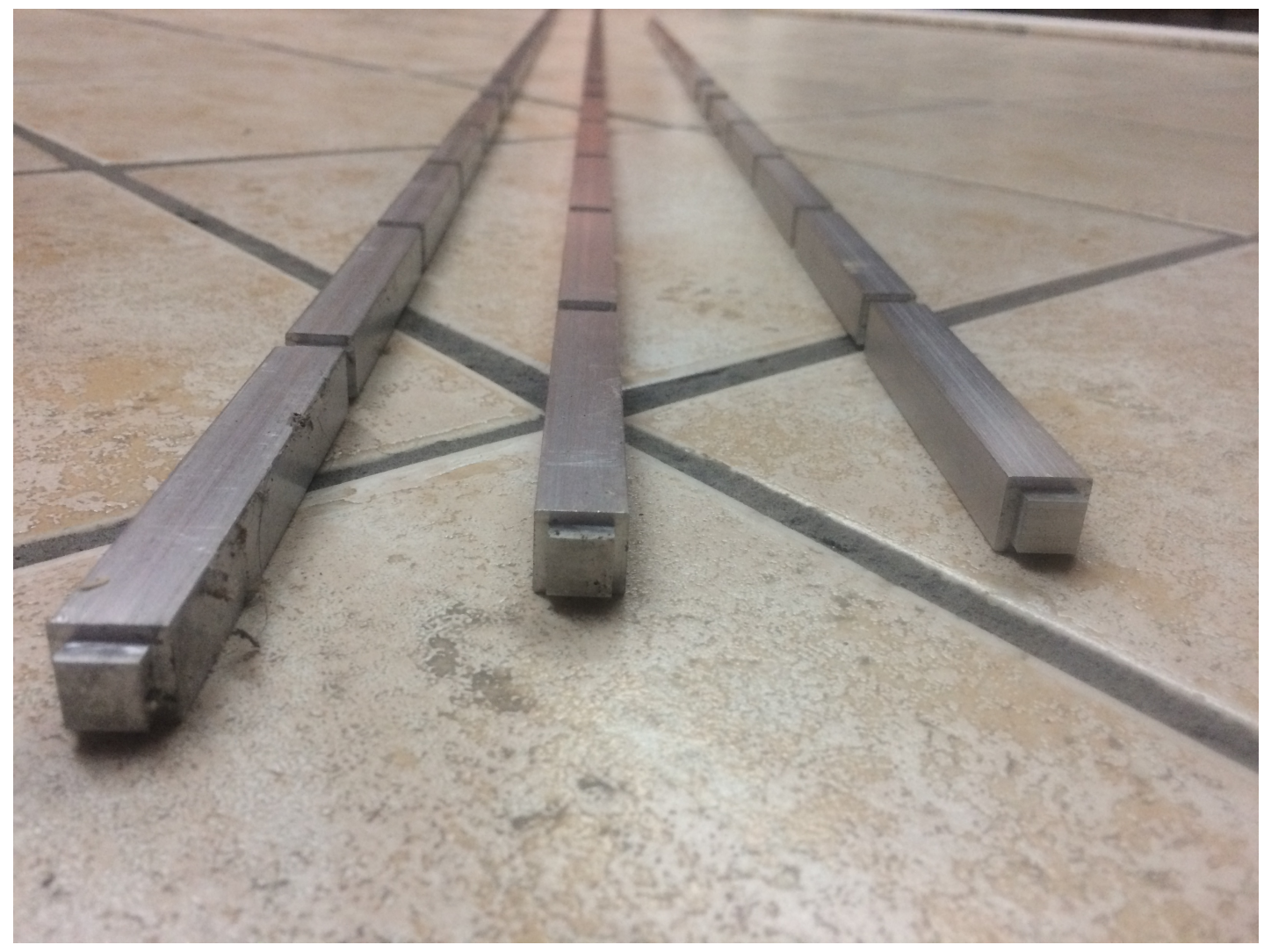

Figura 5.2: Diseños finales de los cristales artificiales elásticos.

\subsection{Técnica de espectroscopia acústica resonante (ARS)}

El montaje experimental para medir los espectros de frecuencias torsionales, de cada sistema elástico, usando la técnica de espectroscopia acústica resonante (ARS), se muestra en la figura 5.3 y se describe a continuación: 1.- Se comienza con la generación de una señal armónica, de frecuencia $f_{0}$ fija, en el analizador de redes vectorial (VNA). 2.- Esta señal se manda a un amplificador de audio (CERWIN-VEGA! 2800), el cual incrementa la potencia de la señal generada. 3.- Posteriormente, esta señal se envía a un transductor electromagnético acústico (EMAT) [7] que, en la cercanía del sistema elástico, (estructura de superceldas acopladas) genera vibraciones mecánicas sin contacto inducida por interacción electromagnética [7] y selectivas en la barra estructurada. 4.- En otra posición de la barra un segundo EMAT detecta, también por inducción electromagnética, la respuesta del sistema a la exitación mécanica y la convierte en una señal de voltaje. 5.-Esta respuesta elástica regresa al VNA para ser comparada con la señal armónica inicial, de excitación de frecuencia $f_{0}$, mencionada en el paso 1 . Los datos son graficados directamente en el VNA; sin embargo, es conveniente usar una computadora PC para el almacenamiento automatizado y ánalisis de los datos de las distintas mediciones. 


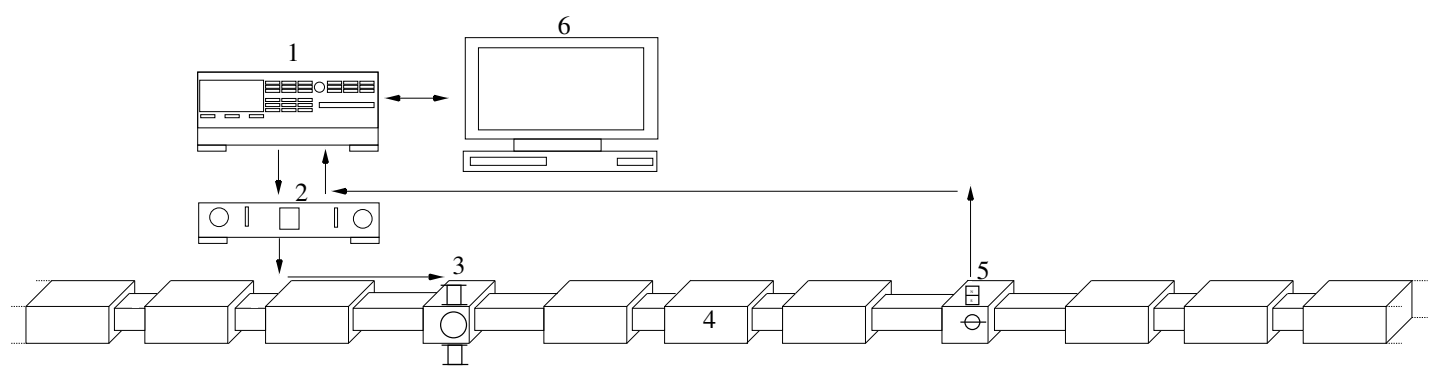

Figura 5.3: Configuración usada para caracterizar las vibraciones de las estructuras elásticas con el método de espectroscopia acústica resonante (ARS). La configuración está compuesta por una estación de trabajo (6), un Vector Network Analizer (Anritsu MB-4630B) (1), un amplificador (Cerwin-Vega CV-2800) de alta fidelidad (2) y dos transductores electromagnéticos acústicos (3 y 5). La estructura elástica caracterizada se indica como (4). La imagen corresponde a dos resonadores elásticos acoplados.

En la figura 5.5 las amplitudes de onda torsionales numéricas sirven como guía para identificar las posiciones de los máximos y mínimos del perfil en el sistema. Para excitar y detectar el espectro de ondas torsionales eficientemente hay que tener en cuenta la localización de las amplitudes de onda en nuestro sistema. La excitación y detección de las ondas torsionales con los transductores tipo EMATs, se hicieron colocando el excitador y el detector en las superceldas del sistema. Para medir los patrones estacionarios asociados a cada frecuencia $f_{0}$ del espectro se fijaron las posiciones del excitador y del detector en los máximos totales (ver figura 5.5).

Manteniendo la frecuencia de exitación y cambiando la posición del EMAT detector, a lo largo del sistema, con la $f_{0}$ constante, es posible detectar las amplitudes de onda, como función de la posición. Posteriormente, se incrementa $f_{0}$ a $f_{0}+\Delta f$ y se repite el procedimiento. 


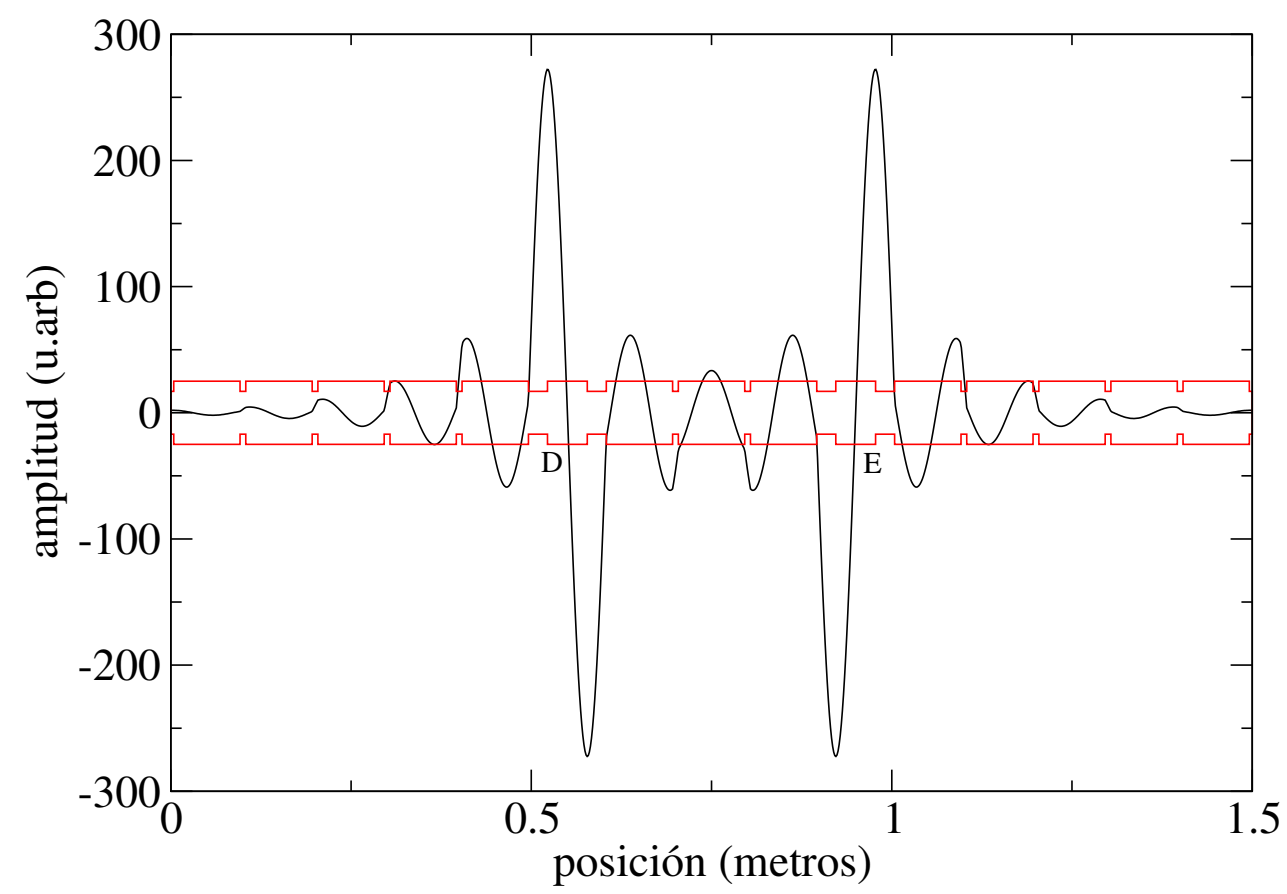

Figura 5.4: Configuración espacial del detector (D) y del excitador (E) en el sistema para medir el espectro de frecuencias torsionales. Se muestra también la amplitud de onda como función de la posición.

\subsection{Resultados experimentales}

\subsubsection{Espectro de frecuencia}

En esta sección se estudian experimentalmente los sistemas elásticos vibrantes formados por unidades elásticas acopladas, previamente diseñados en el capítulo 3. Posteriormente los sistemas fabricados caracterizados por el método de espectroscopia acústica resonante (ARS), descrito en la sección anterior se comparan con los resultados del modelo de enlace fuerte explicado en el capítulo 2.

Las 5 varillas estructuradas de aluminio diseñadas tienen sección transversal cuadrada y consisten de $2,3,4,5$, y 6 superceldas acopladas. Las longitudes totales de cada una son: $1.5,1.9,2.3,2.7$ y $3.1 \mathrm{~m}$, respectivamente (ver diseños en la figura 3.21a). 


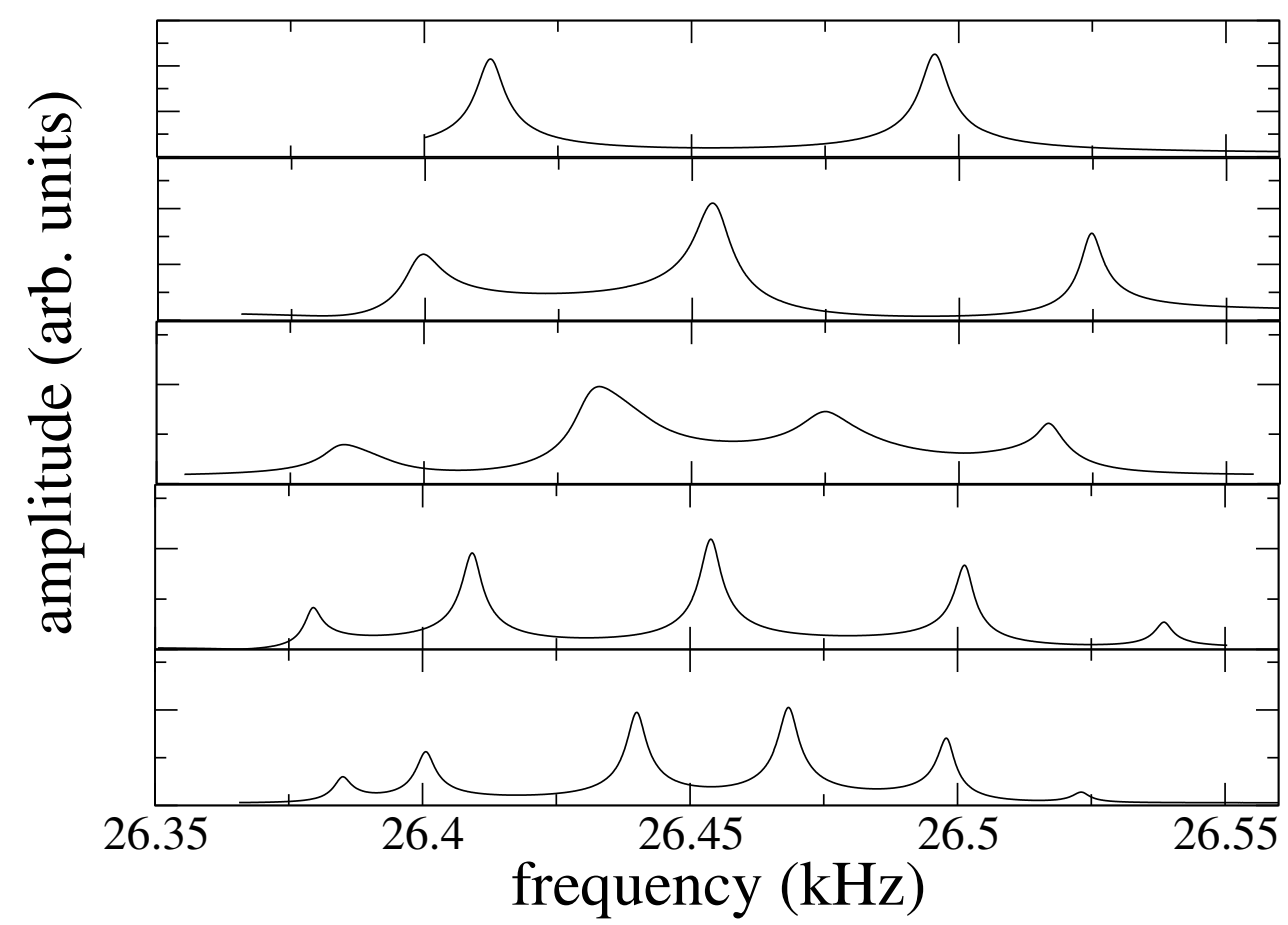

Figura 5.5: Banda de frecuencias experimental, emergente. Medidas realizadas en sistemas de 2 hasta 6 superceldas elásticas acopladas.

Los resultados experimentales se muestran en la figura 5.5. En dicha figura se muestra la respuesta, en frecuencia, de cada uno de estos sistemas, en el intervalo de 26,300 a 26,600 $\mathrm{Hz}$, comprobando el surgimiento de la banda emergente predicha por el modelo de enlace fuerte. Las gráficas de la amplitud como función de la frecuencia se obtuvieron usando el montaje de la figura 5.3, detectando y excitando las señales según la configuración de la figura 5.5 del sistema de 2, 3, 4, 5 y 6 superceldas. Las gráficas de la figura 5.5 indican máxima vibración o respuesta en el centro de cada uno de los defectos de la estructura. Cada gráfca necesita de un tiempo aproximado de medición de media hora. 


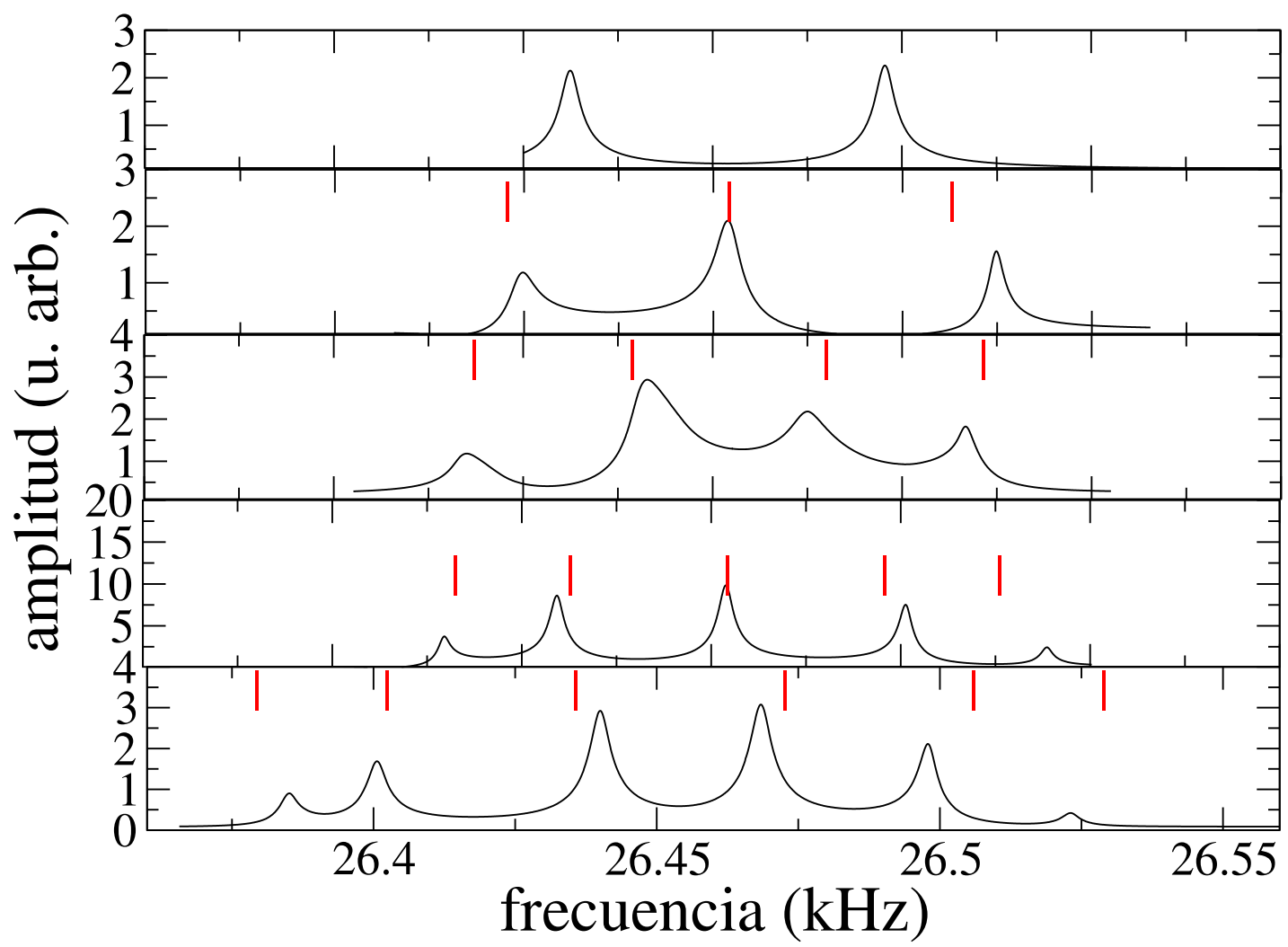

Figura 5.6: Comparación de los resultados experimentales vs modelo empírico. De arriba hacia abajo se muestran las medidas espectrales de la banda torsional emergente, de las barras con 2, 3, 4, 5 y 6 superceldas. Los resultados numéricos del modelo de enlace fuerte propuesto están indicados por líneas rojas verticales.

En la figura 5.6 se observan las resonancias experimentales esperadas. Dichas resonancias se encuentran muy cerca de la predicción numérica, dada por el modelo de acoplamiento empírico en el capítulo 3. La gráfica mostrada en la figura 5.6) muestra el surgimiento de la banda emergente, para ondas torsionales de la estructura elástica localmente periódica medida hasta para 6 superceldas. Se puede ver que los niveles de frecuencia coinciden con los predichos por el modelo empírico de acoplamiento elástico por un error máximo del $0.04 \%$. Esto comprueba que la supercelda propuesta como celda unitaria para construir el cristal elástico emula el comportamiento de un cristal atómico. Esto es un logro importante ya que hemos podido medir, por primera vez en un cristal elástico, una banda de frecuencias torsional, emergente, que exhibe la misma fenomenología que se observa en un cristal atómico.

\subsubsection{Amplitud de onda}

Primero se desarrolló un montaje experimental para medir la amplitud de onda a lo largo de toda la barra. Después se desarrolló un programa en fortran para obtener la 
amplitud de onda a partir de los resultados experimentales. Dicho programa requiere la amplitud y la fase obtenida del VNA. Con esas dos magnitudes graficamos la parte real e imaginaria. El resultado de dichas gráficas es un círculo y el radio nos da la amplitud para cada punto de la barra.

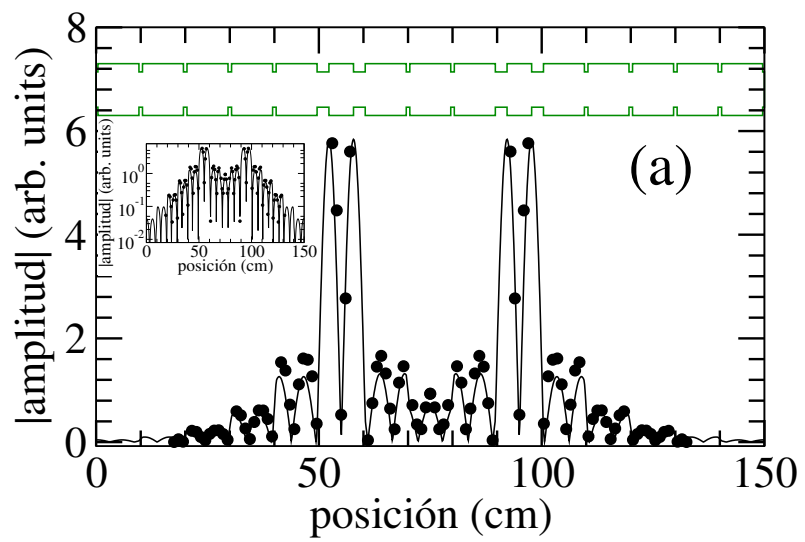

(a)

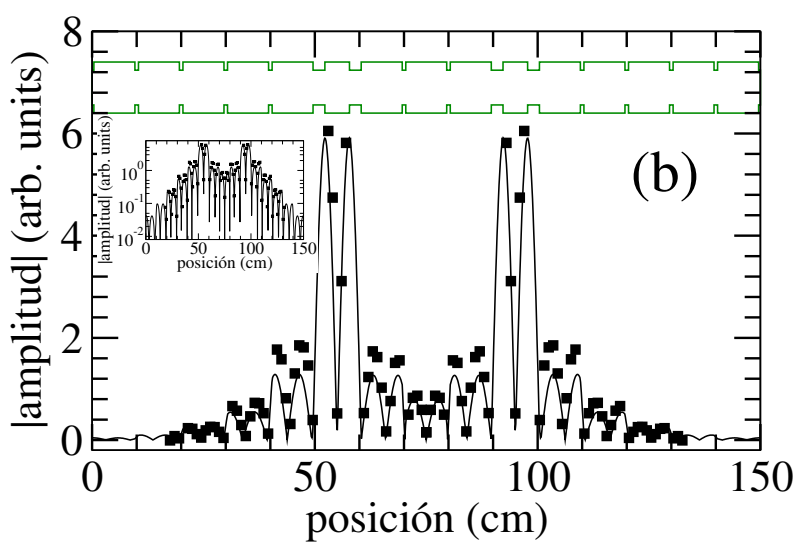

(b)

Figura 5.7: Patrones estacionarios experimentales de la amplitud de onda medidas para el sistema elástico de 2 superceldas acopladas a): Absoluto de la amplitud de onda del patrón estacionario simétrico; b): Amplitud de onda antisimétrica.

En la figura 5.7a) se muestra la comparación de la amplitud de onda numérica, obtenida usando el método de la matriz de transferencia (línea continua), con los resultados experimentales obtenidos por espectroscopia acústica resonante (puntos). En dicha gráfica se muestra el absoluto de la amplitud de onda para el sistema de 2 superceldas acopladas. La primer gráfica (5.7a) es la amplitud asociada a la $f_{-}$y es un patrón simétrico y $5.7 b)$ esta la asociada a la $f_{+}$y es antisimétrica. En las mismas gráficas se muestra el logaritmo de la amplitud de onda vs la frecuencia y se aprecia claramente el decaimiento exponencial. En la parte superior se indica un corte logitudinal del sistema de dos superceldas. Se puede notar que la amplitud de onda tiene máximos alrededor del centro de cada supercelda elástica.

A continuación de la figura 5.8 a la figura 5.11 se muestran las medidas de la amplitud de onda correspondientes hechas con el método de espectroscopia acústica resonante para los sistemas de 3 y hasta 6 superceldas acopladas. Dichas medidas también se comparan con el método de la matriz de transferencia. Dicha comparación se hace a partir de la posición de la segunda celda simple hasta la posición de la última supercelda. Esto es, debido a que en esas posiciones se encontraban los soportes. Para medir toda la barra se tendrían que cambiar las condiciones iniciales. Debido a las limitaciones del VNA sólo se comparó el absoluto de la amplitud de onda de los sistemas desde 3 hasta 6 superceldas. Las amplitudes de onda que se midieron corresponden a las frecuencias dentro del gap que previamente se habían medido y mostrado en la figura 5.6. En el eje y se tiene el absoluto de la altura de la amplitud de onda y en el eje x tenemos la posición en cm. Las 
líneas continuas corresponden al método numérico y los puntos al experimento. En dichas amplitudes de onda se puede ver un decaimiento exponencial.
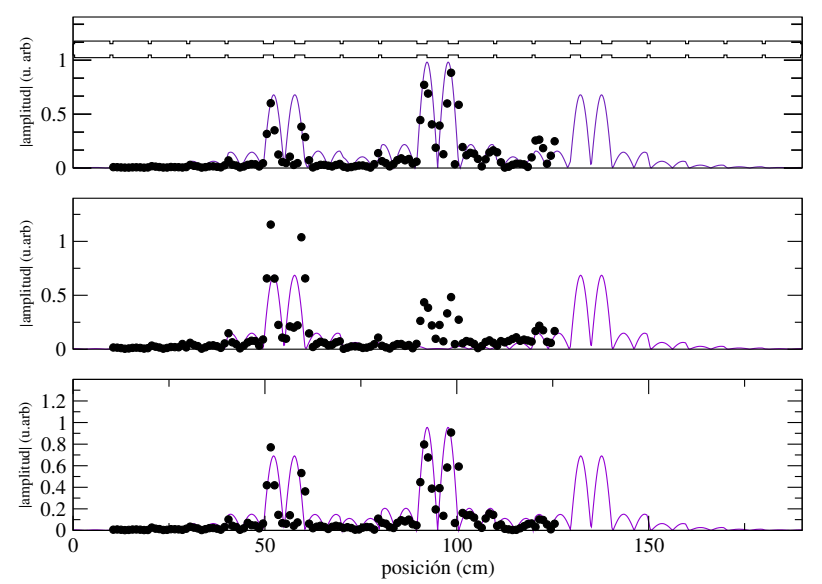

Figura 5.8: Amplitudes de onda asociadas con un sistema de 3 defectos. En orden descentente la comparación de los resultados numéricos con los experimentos de las amplitudes de onda asociadas con el sistema de 3 superceldas acopladas corresponden a los modos 36,37 y 38 de los niveles en el gap, con $f_{36}=26472.237 \mathrm{~Hz}, f_{37}=26544.451 \mathrm{~Hz}$ y $f_{38}=$ $26613.457 \mathrm{~Hz}$, respectivamente son mostrados.

En la figura 5.8 se muestran las amplitudes de onda asociadas al sistema de las 3 superceldas acopladas correspondiente a los modos 36, 37 y 38 de los niveles en el gap, con $f_{36}=26472.237 \mathrm{~Hz}, f_{37}=26544.451 \mathrm{~Hz}$ y $f_{38}=26613.457 \mathrm{~Hz}$, respectivamente (ver figura 5.8). Este sistema es más grande que el anterior debido a que tiene 3 superceldas acopladas. En la gráfica de arriba de la figura 5.8 cada punto experimental coincide con las líneas de MMT en los máximos totales, además el error es menor justo en el segundo acoplador. Lo mismo pasa para la gráfica inferior de la misma figura. En el caso de la figura de en medio el experimento contribuye a dos máximos, uno de ellos ubicado en el segundo defecto, dicho máximo no existe numéricamente, esto se podría deber a una imperfección del material. En las figura 5.8 se puede observar a simple vista que la mayoría de los puntos medidos coinciden con la línea continua obtenidos de la matriz de transferencia. 


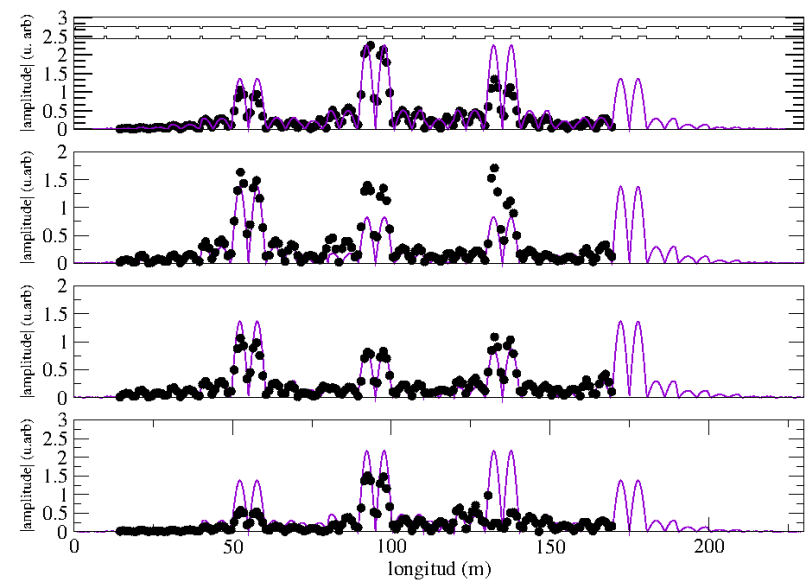

Figura 5.9: Amplitudes de onda asociadas con un sistema de 4 defectos. En orden descentente la comparación de los resultados numéricos con los experimentos de las amplitudes de onda asociadas con el sistema de 4 superceldas acopladas corresponden a los modos $43,44,45$ y 46 de los niveles en el gap, con $f_{43}=26461.323 \mathrm{~Hz}, f_{44}=26512.724 \mathrm{~Hz}$, $f_{45}=26574.426 \mathrm{~Hz}$ and $f_{46}=26622.941 \mathrm{~Hz}$, respectivamente son mostrados.

En la figura 5.9 se muestran la comparación de los resultados numéricos con experimento de las amplitudes de onda asociadas al sistema de las 4 superceldas acopladas correspondiente a los modos $43,44,45$ y 46 de los niveles en el gap, con $f_{43}=26461.323$ $\mathrm{Hz}, f_{44}=26512.724 \mathrm{~Hz}, f_{45}=26574.426 \mathrm{~Hz}$ y $f_{46}=26622.941 \mathrm{~Hz}$, respectivamente (ver figura 5.9). Debido a las limitaciones de potencia de los dispositivos de amplificación en algunas posiciones la amplitud de onda experimental es menor que la numérica, todo esto es debido a la dependencia de la altura con respecto a la potencia. Se midió más del $50 \%$ del sistema total y en la mayoría de las gráficas la posición de las amplitudes de onda experimentales coinciden con las numéricas. 


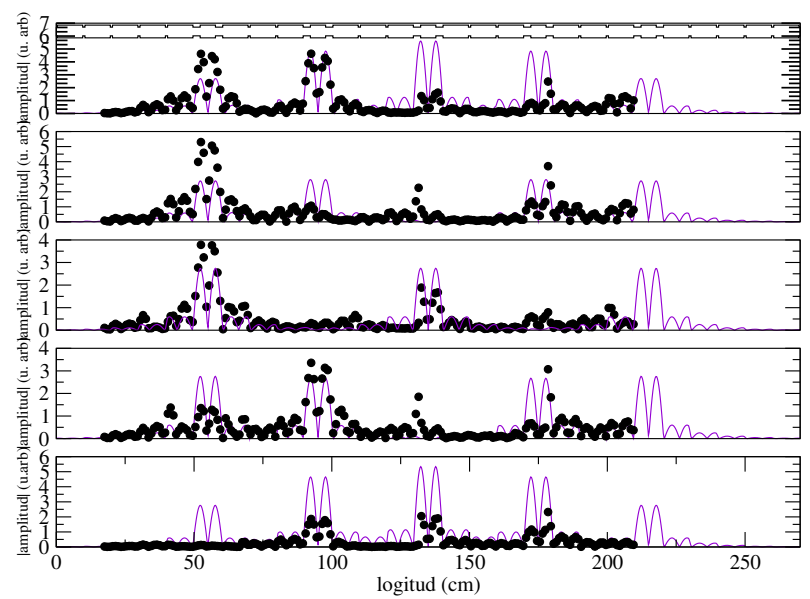

Figura 5.10: Amplitudes de onda asociadas con un sistema de 5 defectos. En orden descentente la comparación de los resultados numéricos con los experimentos de las amplitudes de onda asociadas con el sistema de 5 superceldas acopladas corresponden a los modos 48, 49, 50, 51 y 52 de los niveles en el gap, con $f_{48}=26455.266 \mathrm{~Hz}, f_{49}=26492.932 \mathrm{~Hz}$, $f_{50}=26543.401 \mathrm{~Hz}, f_{51}=26592.791 \mathrm{~Hz}$ and $f_{52}=26628.293 \mathrm{~Hz}$, respectivamente son mostrados.

En la figura 5.10 se muestran las amplitudes de onda asociadas al sistema de las 5 superceldas acopladas correspondientes a los modos 48, 49, 50, 51 y 52 de los niveles en el gap, con $f_{48}=26455.266 \mathrm{~Hz}, f_{49}=26492.932 \mathrm{~Hz}, f_{50}=26543.401 \mathrm{~Hz}, f_{51}=26592.791 \mathrm{~Hz}$ y $f_{52}=26628.293 \mathrm{~Hz}$, respectivamente. Como se puede ver en las figuras hemos conseguido medir la amplitud de onda para un sistema muy grande conservando aún así un excelente acuerdo entre teoría y experimento, esto se ve reflejado en la coincidencia línea-punto del experimento y del método en los máximos totales y en los máximos locales. 


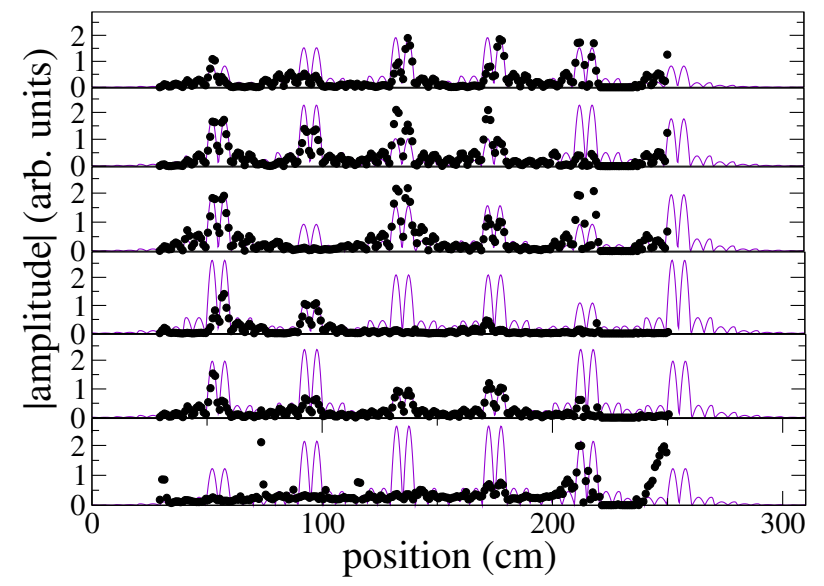

Figura 5.11: Amplitudes de onda asociadas con un sistema de 6 defectos. En orden descentente la comparación de los resultados numéricos con los experimentos de las amplitudes de onda asociadas con el sistema de 6 superceldas acopladas corresponden a los modos 55, $56,57,58,59$ y 60 de los niveles en el gap, con $f_{55}=26451.58 \mathrm{~Hz}, f_{56}=26480.109 \mathrm{~Hz}, f_{57}=$ $26520.79 \mathrm{~Hz}, f_{58}=26565.227 \mathrm{~Hz}, f_{59}=26604.648$ y $f_{60}=26631.598 \mathrm{~Hz}$, respectivamente son mostrados.

Por último en la figura 5.11 se muestra en orden descendente la comparación de las amplitudes de onda numéricas vs experimetnles asociadas al sistema de las 6 superceldas acopladas correspondientes a los modos $55,56,57,58,59$ y 60 de los niveles en el gap, con $f_{55}=26451.58 \mathrm{~Hz}, f_{56}=26480.109 \mathrm{~Hz}, f_{57}=26520.79 \mathrm{~Hz}, f_{58}=26565.227 \mathrm{~Hz}, f_{59}=$ $26604.648 \mathrm{~Hz}$ y $f_{60}=26631.598 \mathrm{~Hz}$, respectivamente. Se puede ver como el experimento reprodujo las amplitudes de onda con máximos alrededor del centro de cada supercelda, excepto la amplitud de onda asociada a la frecuencia $f_{60}=26631.598 \mathrm{~Hz}$. 


\section{Capítulo 6}

\section{Conclusiones}

A lo largo de este estudio se diseñó y calculó numéricamente, usando TMM, el espectro de frecuencias torsional de una supercelda elástica unitaria que nos permitió construir estructuras artificiales localmente periódicas cuya fenomenología es análoga a la de un cristal atómico 1D caracterizada por una de las bandas emergentes de resonancias de interés estan distribuidas en un intervalo de $100 \mathrm{~Hz}$ alrededor de los $25 \mathrm{kHz}$. Este diseño se hizo para obtener experimentalmente su espectro de ondas torsionales y compararlos con el modelo desarrollado y propuesto.

Se calculó el espectro de frecuencias correspondiente a las ondas torsionales para sistemas elásticos desde 1 hasta 10 defectos (superceldas) acoplados. Los resultados obtenidos fueron comparados usando un modelo de enlace fuerte con los obtenidos con el método TM, concluimos que los resultados obtenidos con ambos métodos son muy semejantes (difieren menos del 1\%), pero el tiempo de cálculo es mucho menor usando el de enlace fuerte. Por ello el cálculo numérico del espectro se extendió hasta para 100 impurezas acopladas periódicamente y observamos que los resultados obtenidos satisfacen la relación de dispersión.

Esto se logro a partir de un sistema con estructura periódica, mediante la deformación de una o varias celdas unitarias simples, fue posible generar bandas de frecuencias elásticas en varios gaps del espectro original asociado a la estructura periódica. Sólo se analizó en este estudio la banda emergente obtenida en el segundo gap, debido a las limitaciones de nuestros aparatos, así como al extenso tiempo de mediciones que ello implica. Pudimos constatar que la amplitud de onda obtenida numéricamente para una supercelda aislada sirve como una base; ya que al variar la supercelda aislada, a otra posición del sistema original, la amplitud solo cambia de posición pero mantiene su forma.

Nuestro modelo explicado en el capítulo 5 pudo reproducir la banda emergente obtenida con TMM y la hecho con el ARS. El método de la matriz de transferencia solo puede analizar un sistema de 10 superceldas acopladas, enlace fuerte puede analizar sistemas de hasta más de 1000 superceldas acopladas. Nuestro modelo también nos arroja los eigenvectores. Con dichos datos también podemos obtener la amplitud de onda y compararla con los resultados experimentales.

Al considerar un cristal elástico formado por celdas estructuradas acopladas periódicamente descrito con el método de enlace fuerte, hemos podido reproducir muy bien las 
frecuencias de la banda emergente obtenida experimentalmente con espectroscopia acústica resonante, para un cristal elástico de hasta 6 impurezas, con un error menor al $1 \%$.

Usando una fresadora vertical CNC con control Centroid M400 se fabricaron, en el ICFCuernavaca, 5 sistemas de aluminio de 2 y hasta 6 superceldas acopladas y con sección transversal cuadrada. Dicha construcción tardo alrededor de 5 semanas. Los sistemas también se pueden fabricar con una varilla de sección transversal circular sólo que es más complicado, además no se cuenta con todo el equipo necesario.

Se estudió experimentalmente el acoplamiento por una estructura localmente periódica. El método de acoplamiento permitió que dos amplitudes de onda se acoplaran en el gap y que los niveles se distribuyeran de forma simétrica. Dicho acoplador resultó ser un sistema compuesto por 3 celdas simples. El estudio fue mediante espectroscopia acústica resonante (ARS). Las ondas utilizadas fueron ondas torsionales. Se encontraron resonancias asociadas a las predicciones numéricas usando las configuraciones de los EMATs en modo torsional. Dichos estados son estados localizados, lo que significa que decaen rápidamente a medida que nos alejamos del defecto. Al comparar el modelo elástico empírico, este reproduce muy bien las frecuencias de la banda emergente obtenida experimentalmente con espectroscopía acústica resonante con un error menor al $1 \%$.

Se midió la amplitud de onda asociada a los diseños mostrados en la figura 3.21b. La amplitud de onda medida para un sistema de dos superceldas nos sirvió para explicar el efecto túnel clásico. Dicho trabajo se publicó en las memorias del congreso PIERS. En cuanto a las amplitudes de onda medidas para los otros sistemas nos sirvió para demostrar que dichos estados pertenen al gap. Mostramos teórica y experimentalmente que para una barra localmente periódica ,con dos defectos localizados simétricamente con respecto al centro de dicho sistema, es posible localizar dos amplitudes (una simétrica y otra antisimétrica) de onda alrededor de los defectos.

En este trabajo hemos explicado el fenómeno de la localización de modos y la repulsión de niveles mediante el uso de las vibraciones elásticas en una barra. Asimismo hemos presentado, por primera vez, el efecto túnel clásico en elasticidad. Dicho efecto se ha estudiado mucho desde el punto de vista cuántico y muy poco clasicamente.

En este trabajo la tunelización ha sido emulada a través del acoplamiento evanescente mediante una estructura mecánica localmente periódica, que consiste en un número finito de celdas simples. Para esto se hizo una simulación con el método numérico llamado matriz de transferencia MMT, donde escogimos los estados que se encontraban en la brecha. Dichos estados eran los primeros estados excitados del sistema total. Para poderlos medir experimentalmente escogimos que la repulsión fuera fuerte. Dicho fenómeno fue comprobado experimentalmente, mediante la medición de la respuesta del sistema cada cm. a lo largo de toda la barra. Apesar de que el método está hecho para un sistema unidimensional, nuestras medidas experimentales fueron satisfactorias. Recordemos que nuestro sistema es cuasiunidimensional.

Los resultados de este trabajo permiten estudiar u observar en sistemas clásicos y en escalas macroscópicas cierta fenomenología de sistemas o cristales atómicos en el experimento reduciendo costos. 


\section{Bibliografía}

[1] A.W. Leissa, Vibration of Plates Vol. 1 (Acoustical Society of America, Woodbury, NY, 1993).

[2] W. Soedel, Vibrations of Shells and Plates(Marcel Dekker, New York, 1993).

[3] R. Szilard, Theory and Analysis of Plates: Classical and Numerical Methods (Prentice-all, Englewood Cliffs, NJ, 1974).

[4] D.J. Gorman, Vibration Analysis of Plates by the Superposition Method, Series on Stability Vibrations and Control of Systems, Vol. 3 (World Scientific, Singapore, 1999).

[5] N.S. Bardell, J. Sound Vib. 174, 655-676 (1994).

[6] A. Morales, J. Flores, L. Gutiérrez y R.A. Méndez- Sánchez, J. Acoust. Soc. Am. 112, 1961-1967 (2002).

[7] Filiberto Ramírez Ramírez, Tesis de Licenciatura, Universidad Autónoma Metropolitana (2012).

[8] A. Díaz-de-Anda, J. Flores, L. Gutiérrez, R. A. Méndez-Sánchez, G. Monsivais, and A. Morales, J. Sound Vib. 332, 5732-5744 (2012).

[9] G. Monsivais, R.A. Méndez-Sánchez, A. Díaz-de-Anda, J. Flores, L. Gutiérrez, and Morales, Journal of Mechanics and structures 2, 1629-1638, (2007).

[10] A. Díaz-de-Anda, J. Flores, L. Gutiérrez, R. A. Méndez-Sánchez, G. Monsivais, and A. Morales, J. Sound Vib. 332, 5732-5744 (2012).

[11] J. A. Franco-Villafañe, E Flores-Olmedo, G. Baéz, O. Gandarilla-Carrillo and R. A. Méndez-Sánchez, Eur. J. Phys. 33, 1761-1769 (2012).

[12] Filiberto Ramírez Ramírez, Tesis de maestría: Estudio experimental de ondas elásticas dentro del plano en sistemas periódicos bidimensionales, Universidad Autónoma Metropolitana (2015).

[13] Enrique Flores, Tesis de Doctorado: Medición del transporte ondulatorio en cavidades caóticas elásticas, Universidad Autónoma Metropolitana. 
[14] Román Trejo, Tesis de Maestría: Medición y caracterización de estados atrapados en una guía de onda doblada, Universidad Autónoma Metropolitana (2013).

[15] A. Morales, R. A. Méndez-Sánchez, J. Flores. Physica E 19, 289 (2003).

[16] Kittel, C. Introduction to Solid State Physics (Wiley, 2005).

[17] Ashcroft, N. W. \& Mermin, N. D. Solid State Physics (Hold, Reinhart \& Winston, 1976).

[18] Marco $\breve{s}$, P. \& Soukoulis, C. M. Wave propagation: from electrons to photonic crystals and left-handed materials. (Princeton University Press, 2008).

[19] Poli, Ch., Bellec, M., Kuhl, U., Mortessagne, F. \& Schomerus, H. Selective enhancement of topologically induced interface states in a dielectric resonator chain. Nat. Commun. 6, 1-5 (2015).

[20] Bittner, S. et al. Bound states in sharply bent waveguides: Analytical and experimental approach. Phys. Rev. E 87, 042912 (2013).

[21] Rivera-Mociños, E. and Sadurní, E. Inverse lattice design and its application to bent waveguides. J. Phys. A: Math. Theor. 49, 175302 (2016).

[22] Mattarelli, M., Secchi, M. \& Montagna, M. Phononic crystals of spherical particles: a tight binding approach. J. Chem. Phys. 139, 174710 (2013).

[23] Sainidou, R., Stefanou, N. \& Modinos, A. Linear chain of weakly coupled defects in a three-dimensional phononic crystal: A model acoustic waveguide. Phys. Rev. B 74, 172302 (2006).

[24] Kushwaha, M. S., Halevi, P., Dobrzynski, L. \& Djafari-Rouhani, B. Acoustic band structure of periodic elastic composites. Phys. Rev. Lett. 71, 2022-20025 (1993).

[25] Sigalas, M. M. \& Economou, E. N. Band structure of elastic waves in two dimensional systems. Solid State Commun. 86, 141-143 (1993).

[26] Munday, J. N., Brad Bennett, C. \& Robertson, W. W. Band gaps and defects modes in periodically structured waveguides. J. Acoust. Soc. Am. 112, 1353-1358 (2002).

[27] Khelif, A. et al. Trapping and guiding of acoustic waves by defect modes in a fullband-gap ultrasonic crystal. Phys. Rev. B 68, 214301 (2003).

[28] Jianping Ge, Yadong Yin, Angewandte Chemie International Edition 50, 1492-1522 (2011).

[29] Gang Wang, Xisen Wen, Jihong Wen, Lihui Shao, and Yaozong Liu, Phys. Rev. Lett. 93, 154302 (2004). 
[30] Martin Maldovan, Nature 503, 209-217 (2013)

[31] Tai-I Weng and G. Y. Guoa, J. Appl. Phys 99, 093102 (2006).

[32] Edson Jansen Pedrosa de Miranda Júnior and José Maria Campos Dos Santos, Materials Research 20, 555-571 (2007).

[33] Francisco Javier López Rodríguez, Tesis de Doctorado: Transporte de carga en grafeno bajo radiación electromagnética, Universidad Nacional Autónoma de México (2010).

[34] Omar Vásquez Candanedo, Tesis de Doctorado, Universidad Nacional Autónoma de México (2015).

[35] J.A. Franco-Villafañe, E. Sadurní, S. Barkhofen, U. Kuhl, F. Mortessagne, and T.H. Seligman, Phys. Rev. Lett. 111, 170405 (2013).

[36] T Hartamann, F Keck, H J Korsch and S Mossmann, New Journal of Physics 6, 1367-2630 (2004).

[37] https://www.cond-mat.de/events/correl16/manuscripts/foulkes.pdf

[38] Gabriel Queiroz Garcia, Tesis de maestría, Universida de Federal da Paraíba (2013).

[39] H. Herrera y César Mora, Revista Mexicana de Física 52, 534-539 (2006).

[40] Julian Böhm, Matthieu Bellec, Fabrice Mortessagne, Ulrich Kuhl, Sonja Barkhofen, Stefan Gehler, Hans-Jürgen Stöckmann, Iain Foulger, Sven Gnutzmann and Gregor Tanner. arXiv.org > cond-mat > arXiv:1409.2382.

[41] E. Sadurni and W.P Scheleich. Conformal mapping and bound states in bent waveguides. AIP, 1323:283-295, 2010.

[42] J Goldstone and R.L. Jaffe. 14100. Phys Rev. , B45:3, (1992).

[43] J. Carini, J. T. Londergan and D. P. Murdock. 9842. Phys. Rev., B 55:3, (1997).

[44] J. Carini, J. T. Londergan, Kieran Mollen, and D. P. Murdock. 15538. Phys. Rev., B 46:2, (1992).

[45] Ulrich Kuhl, Gilles Montambaux, and Fabrice Mortessagne, Phys. Rev. B 88, 115437 (2013).

[46] Shanjin He and J. D. Maynard, Phys. Rev. Lett. 62, 16 (1989).

[47] S. Hossein Mousavi, Alexander B. Khanikaev \& Zheng Wang, Nature Communications 6, 8682 doi:10.1038/ncomms9682. 
[48] Xiao, M., Chen, W.-J., He, W.-Y., Zhang, Z. Q. \& Chan. Preprint at http://arxiv.org/abs/1503.06295 (2015).

[49] Apuntes de física del estado sólido. Alberto Rubio Ponce.

[50] Matthieu Bellec, Ulrich Kuhl, Gilles Montambaux, and Fabrice Mortessagne, Phys. Rev. B 88, 115437 (2013).

[51] J.A. Franco-Villafañe, E. Sadurní, S. Barkhofen, U. Kuhl, F. Mortessagne and T.H. Seligman, Phys. Rev. Lett. 111, 170405 (2013).

[52] Charles Poli, Matthieu Bellec, Ulrich Kuhl, Fabrice Mortessagne and Henning Schomerus, nature comunicatios 7710, (2015).

[53] Shanjin He and J. D. Maynard, Phys. Rev. Lett. 62, No. 16 (1989).

[54] Víctor Rodríguez Rocha, Tesis de Doctorado, Universidad Autónoma Metropolitana (2014).

[55] Thomas D. Rossing and Neville H. Fletcher. Principles of Vibration and sound (Springer, second edition). 


\section{Apéndice A}

\section{Matriz de transferencia}

\section{A.1. Matriz de transferencia}

Un ejemplo de una barra que nos va ayudar a enteder como funciona el método de la matriz de transferencia es la que se muestra en la figura A.1.

En la figura A.1 se tiene una barra que tiene $\mathrm{n}$ cilindros arreglados de forma periodica. Cada celda esta formada por tres cilindros. El radio de la barra es $\mathrm{R}$ y tiene n-1 muescas. Hay dos secciones transversales una con un radio $\mathrm{r}$ y otra con un radio $\mathrm{R}$, denotadas s y $\mathrm{S}$ respectivamente. La amplitud de onda en el cilindro $i$ es

$$
\psi_{i}(z)=A_{i} e^{i k\left(z-z_{i-1}\right)}+B_{i} e^{-i k\left(z-z_{i-1}\right)},
$$

con $z_{i-1} \leq z \leq z_{i}, i=1,2, \ldots, 2 n+1$, para ondas torsionales la condición de continuidad de la función de onda es:

$$
\left.\psi_{i}\right|_{z i}=\left.\psi_{i+1}\right|_{z_{i}}
$$

y la condición de continuidad para el momento torsional es:

$$
\left.s_{i}^{2} \frac{\partial \psi_{i}}{\partial z}\right|_{z i}=\left.s_{i+1}^{2} \frac{\partial \psi_{i+1}}{\partial z}\right|_{z_{i}} .
$$

Teniendo en cuenta las ecuaciones A.2 y A.3 la matriz de transferencia que une los coeficientes de amplitud de onda $A_{i+1}$ y $B_{i+1}$ para $A_{i}$ y $B_{i}$ es dado para ondas torsionales por

$$
\left(\begin{array}{c}
A_{i+1} \\
B_{i+1}
\end{array}\right)=M_{i \rightarrow i+1}\left(\begin{array}{c}
A_{i} \\
B_{i},
\end{array}\right)
$$

con 


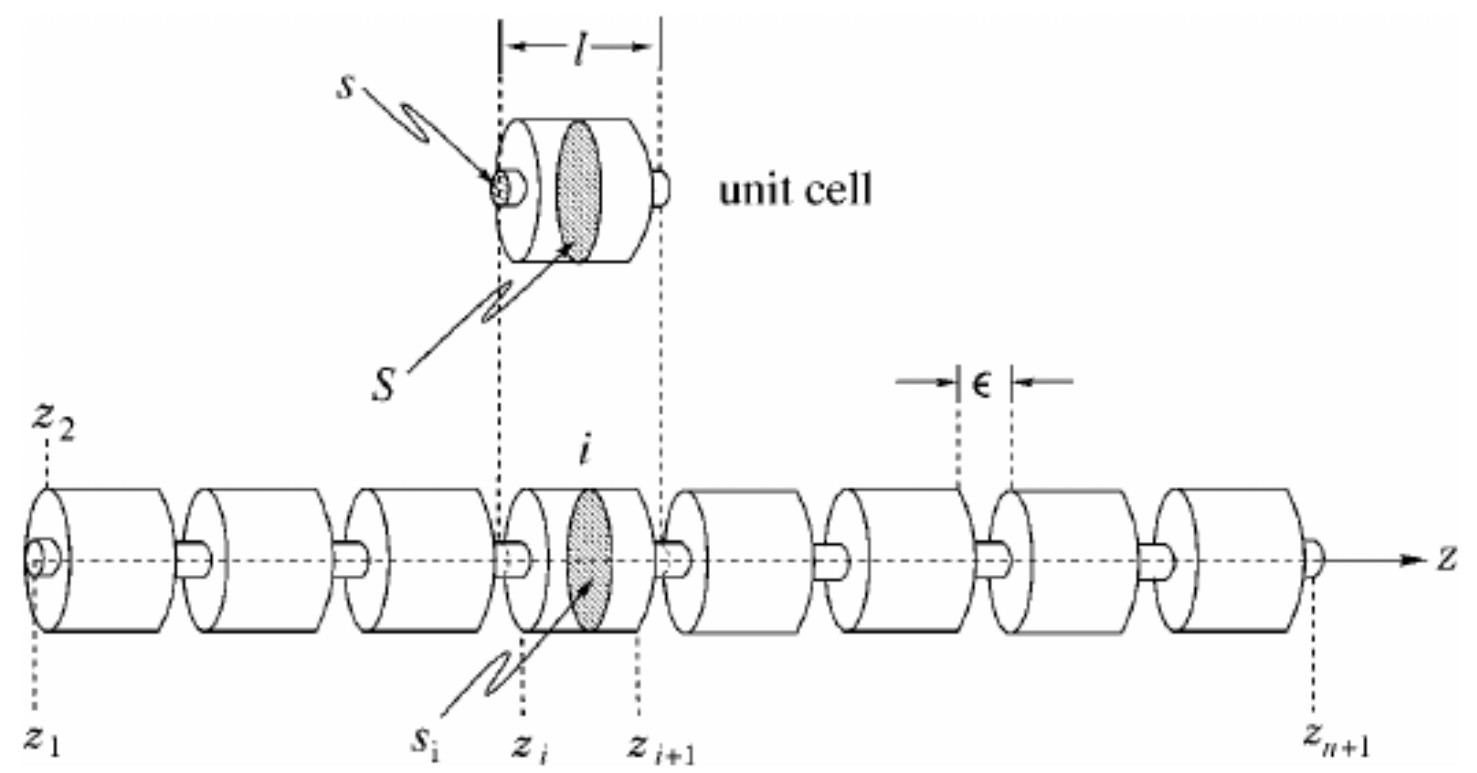

Figura A.1: Geometría de la varilla con n celdas y (n-1) muescas. Se denota con $z_{i}$ la posición donde cambia el radio y con $s_{i}$ el área transversal del i-ésimo cilindro de radio $r_{i} . z_{0}=0$ y $z_{2 n+1}=L$.

$$
M=M_{i \rightarrow i+1}\left(\begin{array}{ll}
t^{\prime}-\frac{r r^{\prime}}{t} & \frac{r}{t} \\
\frac{-r^{\prime}}{t} & \frac{1}{t}
\end{array}\right),
$$

además $r+t=1$ y $r^{\prime}+t^{\prime}=1$, por lo tanto

$$
M=M_{i \rightarrow i+1}\left(\begin{array}{ll}
1-\frac{r^{\prime}}{1-r} & \frac{r}{1-r} \\
\frac{-r^{\prime}}{1-r} & \frac{1}{1-r}
\end{array}\right) .
$$

De aquí

$$
M_{i \rightarrow i+1}=1 / 2\left(\begin{array}{ll}
{\left[1+\left(\frac{r_{i}}{r_{i}+1}\right)^{4}\right] e^{i k\left(z_{i}-z_{i-1}\right)}} & {\left[1-\left(\frac{r_{i}}{r_{i+1}}\right)^{4}\right] e^{-i k\left(z_{i}-z_{i-1}\right)}} \\
{\left[1-\left(\frac{r_{i}}{r_{i+1}}\right)^{4}\right] e^{i k\left(z_{i}-z_{i-1}\right)}} & {\left[1+\left(\frac{r_{i}}{r_{i+1}}\right)^{4}\right] e^{-i k\left(z_{i}-z_{i-1}\right)}}
\end{array}\right)
$$

Al componer $2 \mathrm{n}+1$ matrices de transferencia, los coeficientes $A_{2 n+1}$ y $B_{2 n+1}$ se expresan en términos de $A_{1}$ y $B_{1}$ :

$$
\begin{gathered}
\left(\begin{array}{c}
A_{2 n+1} \\
B_{2 n+1}
\end{array}\right)=M\left(\begin{array}{c}
A_{1} \\
B_{1}
\end{array}\right) \\
M=M_{2 n \rightarrow 2 n+1} M_{2 n-1 \rightarrow 2 n} \ldots M_{2 \rightarrow 3} M_{1 \rightarrow 2} .
\end{gathered}
$$


En esta situación tenemos condiciones de frontera de Neumann, es decir una varilla con extremos libres para el caso torsional. Por un lado tenemos $z=0$. Para esto tomamos la ecuación A.1 es decir,

$$
\begin{gathered}
\psi_{i}(0)=A_{1} e^{i k\left(0-z_{1-1}\right)}+B_{1} e^{-i k\left(0-z_{1-1}\right)} \\
\psi_{i}(0)=A_{1} e^{i k\left(0-z_{0}\right)}+B_{1} e^{-i k\left(0-z_{0}\right)} \\
\psi_{i}(0)=A_{1} e^{i k(0-0)}+B_{1} e^{-i k(0-0)} \\
\psi_{i}(0)=A_{1} e^{i k 0}+B_{1} e^{-i k 0} \\
\psi_{i}(0)=A_{1}+B_{1} \\
0=A_{1}+B_{1} .
\end{gathered}
$$

Ahora para $z=z_{2 n+1}=L$ tenemos que

$$
\begin{gathered}
\psi_{2} n+1(L)=A_{2 n+1} e^{i k\left(L-z_{2 n+1-1}\right)}+B_{2} n+1 e^{-i k\left(L-z_{2 n+1-1}\right)} \\
\psi_{2} n+1(L)=A_{2 n+1} e^{i k\left(L-z_{2 n}\right)}+B_{2} n+1 e^{-i k\left(L-z_{2 n}\right)} \\
0=A_{2 n+1} e^{i k\left(L-z_{2 n}\right)}+B_{2} n+1 e^{-i k\left(L-z_{2 n}\right)}
\end{gathered}
$$

sustituyendo la ecuación A.4 en A.14, además de A.11 se tiene: primero

$$
M=\left(\begin{array}{ll}
M_{11} & M_{12} \\
M_{21} & M_{22}
\end{array}\right),
$$

luego

$$
\left(\begin{array}{l}
A_{2 n+1} \\
B_{2 n+1}
\end{array}\right)=\left(\begin{array}{ll}
M_{11} & M_{12} \\
M_{21} & M_{22}
\end{array}\right)\left(\begin{array}{c}
A_{1} \\
B_{1}
\end{array}\right) .
$$


Después

$$
A_{2 n+1}=A_{1} M_{11}+B_{1} M_{12} B_{2 n+1}=A_{1} M_{21}+B_{1} M_{22} .
$$

Ahora si sustituimos en A.14

$$
\begin{gathered}
\left(A_{1} M_{11}+B_{1} M_{12}\right) e^{i k\left(L-z_{2 n}\right)}-\left(A_{1} M_{21}+B_{1} M_{22}\right) e^{-i k\left(L-z_{2 n}\right)}=0 \\
A_{1} M_{11} e^{i k\left(L-z_{2 n}\right)}+B_{1} M_{12} e^{i k\left(L-z_{2 n}\right)}-A_{1} M_{21} e^{-i k\left(L-z_{2 n}\right)}-B_{1} M_{22} e^{-i k\left(L-z_{2 n}\right)}=0 \\
A_{1} M_{11} e^{i k\left(L-z_{2 n}\right)}-A_{1} M_{21} e^{-i k\left(L-z_{2 n}\right)}+B_{1} M_{12} e^{i k\left(L-z_{2 n}\right)}-B_{1} M_{22} e^{-i k\left(L-z_{2 n}\right)}=0 .
\end{gathered}
$$

De aquí se obtienen dos ecuaciones homogeneas para $A_{1}$ y $B_{1}$

$$
P\left(\begin{array}{l}
A_{1} \\
B_{1}
\end{array}\right)=\left(\begin{array}{l}
0 \\
0
\end{array}\right)
$$

con

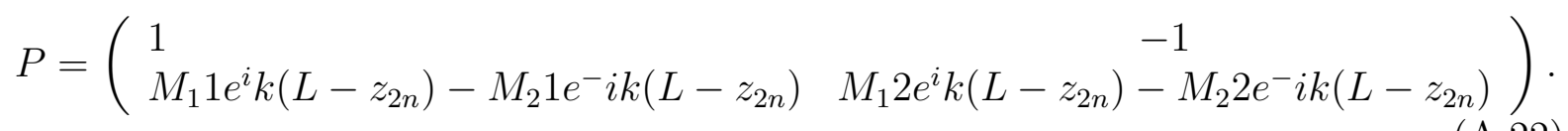

Los eigenvalores de los modos normales corresponden a las raices $\operatorname{del} \operatorname{det}(P)=0$. Los números de onda permitidos se obtienen numéricamente mediante el barrido del determinante en función de $k$. 